\title{
A 2-D Hybrid Euler-Compressible Vortex Particle Method For Transonic Rotorcraft Flows
}

\author{
by Gregory S. Oxley, B.Eng. \\ A thesis submitted to the Faculty of Graduate Studies and Research in partial \\ fulfillment of the requirements for the degree of \\ Doctorate of Philosophy \\ Ottawa-Carleton Institute for Mechanical and Aerospace Engineering \\ Faculty of Engineering \\ Department of Mechanical and Aerospace Engineering \\ Carleton University \\ Ottawa, Ontario, Canada, K1S 5B6
}

July 2009

(C) Copyright by Gregory S. Oxley, 2009 


\begin{tabular}{|c|c|}
\hline $\begin{array}{l}\text { Library and Archives } \\
\text { Canada }\end{array}$ & $\begin{array}{l}\text { Bibliothèque et } \\
\text { Archives Canada }\end{array}$ \\
\hline $\begin{array}{l}\text { Published Heritage } \\
\text { Branch }\end{array}$ & $\begin{array}{l}\text { Direction du } \\
\text { Patrimoine de l'édition }\end{array}$ \\
\hline $\begin{array}{l}395 \text { Wellington Street } \\
\text { Ottawa ON K1A ON4 } \\
\text { Canada }\end{array}$ & $\begin{array}{l}\text { 395, rue Wellington } \\
\text { Ottawa ON K1A ON4 } \\
\text { Canada }\end{array}$ \\
\hline
\end{tabular}

Your file Votre reférence

ISBN: 978-0-494-60107-5

Our file Notre reference

ISBN: 978-0-494-60107-5

NOTICE:

The author has granted a nonexclusive license allowing Library and Archives Canada to reproduce, publish, archive, preserve, conserve, communicate to the public by telecommunication or on the Internet, loan, distribute and sell theses worldwide, for commercial or noncommercial purposes, in microform, paper, electronic and/or any other formats.

The author retains copyright ownership and moral rights in this thesis. Neither the thesis nor substantial extracts from it may be printed or otherwise reproduced without the author's permission.
AVIS:

L'auteur a accordé une licence non exclusive permettant à la Bibliothèque et Archives Canada de reproduire, publier, archiver, sauvegarder, conserver, transmettre au public par télécommunication ou par l'Internet, prêter, distribuer et vendre des thèses partout dans le monde, à des fins commerciales ou autres, sur support microforme, papier, électronique et/ou autres formats.

L'auteur conserve la propriété du droit d'auteur et des droits moraux qui protège cette thèse. $\mathrm{Ni}$ la thèse ni des extraits substantiels de celle-ci ne doivent être imprimés ou autrement reproduits sans son autorisation.
In compliance with the Canadian Privacy Act some supporting forms may have been removed from this thesis.

While these forms may be included in the document page count, their removal does not represent any loss of content from the thesis.
Conformément à la loi canadienne sur la protection de la vie privée, quelques formulaires secondaires ont été enlevés de cette thèse.

Bien que ces formulaires aient inclus dans la pagination, il n'y aura aucun contenu manquant.

\section{Canadä}




\section{Abstract}

In the present work, a novel hybrid Euler-compressible vortex particle method is developed for 2-D transonic rotorcraft applications. It is identified that the advantages and disadvantages of traditional grid-based Euler/Navier-Stokes methods and those of vortex particle methods complement each other. This suggests that a hybrid approach, in which a grid-based Euler domain covering the near-field around the airfoil, coupled with a compressible vortex particle formulation representing the far-field, would be extremely beneficial to employ.

This work focuses on two areas: firstly, a compressible vortex particle method (CVPM) formulation is developed. Then, the coupling of this method with an existing Euler solver (CMB) is completed, using a domain decomposition technique featuring complete overlap. This novel hybrid Euler-particle method is then designated as HEPM.

Validation of HEPM is performed in three phases. First, the convergence of the method is examined for steady flows at low and high Mach numbers and also for a rapidly sinusoidally pitching airfoil. Then, validation against experimental results is performed by comparisons to the AGARD CT6 and CT5 cases for ramped-up and sinusoidally pitching airfoils, respectively. Finally, airfoil-vortex interaction (AVI) studies are performed for an impinging vortex, with various vortex miss distances considered at both low and high Mach numbers. All of the above cases represent typical problems in rotorcraft aerodynamics. 


\section{Acknowledgements}

This thesis would not be complete without acknowledging the people and organizations involved in all aspects of my research work. Firstly, I'd like to acknowledge NSERC CRD, SRO and OCE for their financial support of this project. Secondly, I'd like to thank my advisors Professor Fred Nitzsche and Professor Daniel Feszty at Carleton University. Their tireless searches for research funding and their connections for enabling study-abroad experiences made my time at Carleton a truly wonderful experience. I'd like to also thank the members of the Carleton Rotorcraft Research group (new and old) whose sense of humour created several episodes which I only remember as "classics".

Next I must thank Professor Spyros Voutsinas at the National Technical University of Athens (NTUA), whose technical support was invaluable to the project. He generously provided lodging during my stays and I will remember our late night technical meetings in Athens well. I must also mention my friends at NTUA: Vasilis R., Giannis, John, Vasilis T., Dennis and Papis who made me feel at home in Greece. I hope all of my friends at NTUA can travel to Ottawa one day, so that I may return the same courtesy.

Last but not least, I must extend many thanks to my lovely wife Rebecca; whose love and support were key to the success of my graduate studies. I am reminded everyday of why I married her. 


\section{Table of Contents}

Abstract $\quad$ iii

Acknowledgements iv

List of Tables $\quad$ x

List of Figures $\quad$ xii

List of Symbols $\quad$ xix

List of Abbreviations $\quad$ xxiv

1 Introduction. $\quad 1$

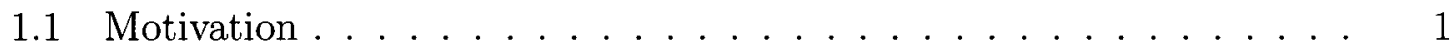

1.2 History of Rotorcraft Aerodynamic Modeling . . . . . . . . . . . . . . 7

1.2.1 Blade-Element Theory . . . . . . . . . . . . 7

1.2.2 Prescribed Wake Models . . . . . . . . . . . . . . . . . . 9

1.2.3 Free Wake Methods . . . . . . . . . . . . . . . 11 
1.2.4 Euler/Navier-Stokes CFD ............... 14

1.3 Vortex particle methods . . . . . . . . . . . . . . . 17

1.3.1 Fast Summation Techniques . . . . . . . . . . 19

1.3.2 Viscous effects . . . . . . . . . . . . . . 22

1.3.3 Compressibility effects ................ 23

1.4 Hybrid methods . . . . . . . . . . . . . . 25

1.5 Thesis Objectives . . . . . . . . . . . . . . . . . . 28

1.5.1 Thesis Outline.................... 29

2 The Compressible Vortex Particle Method Formulation 31

2.1 Euler Equations and the Helmholtz Decomposition . . . . . . . . 31

2.2 Review of Compressible Formulations . . . . . . . . . . . 33

2.2.1 Meske's $(\omega, \phi, p)$ Formulation ............. 33

2.2.2 Eldredge's Dilating Vortex Particle Method (DVPM) . . . . 37

2.2.3 Mas-Gallic's $(\rho, \omega, \phi)$ Formulation . . . . . . . . . . . 39

2.3 Current method: $(\rho, \omega, \phi)$ Formulation . . . . . . . . . . . 39

2.4 Particle Approximation . . . . . . . . . . . . . . . 42

2.5 Particle-Mesh Technique . . . . . . . . . . . . . 48

2.5.1 Projection/Interpolation Operators . . . . . . . . . . 49

2.5.2 Hamilton-Jacobi Equation ............. 51

2.6 CVPM Algorithm . . . . . . . . . . . . . . . 52 
3 Finite-Volume Euler Solver

3.1 Euler Equations . . . . . . . . . . . . . . . . . . . . 55

3.2 Time and Space Discretization . . . . . . . . . . . 56

4 Hybrid Euler-Particle Method (HEPM) $\quad 60$

4.1 HEPM Model . . . . . . . . . . . . . . . . . . . . 60

4.2 Coupling Algorithm . . . . . . . . . . . . . . 63

$4.2 .1 \quad$ Euler Solution Integration $\ldots \ldots \ldots \ldots 6$

4.3 Non-dimensional Hamilton-Jacobi Equation $\ldots \ldots \ldots \ldots$

4.4 Non-Reflective Boundary Conditions . . . . . . . . . . . 70

$\begin{array}{lll}5 & \text { Verification and Validation } & 76\end{array}$

5.1 Verification ..................... 77

5.1 .1 Steady Flow Convergence $\ldots \ldots \ldots \ldots \ldots$

5.1 .2 Rapidly Pitching Airfoil . . . . . . . . . . . . . . 84

5.2 Validation ...................... 96

5.2.1 AGARD CT6 - Ramping Pitch Motion . . . . . . . . . 96

5.2.2 AGARD CT5 - Sinusoidal Pitch Motion . . . . . . . . . 99

5.3 Airfoil-Vortex Interaction . . . . . . . . . . . . . . . . . 104

5.3.1 Low Mach Number AVI Simulation (AVI1) . . . . . . . 108

5.3.2 High Mach Number AVI Simulation (AVI2) $\ldots \ldots \ldots \ldots$ 
6 Conclusions and Recommendations

6.1 Conclusions ........................ 116

6.2 Recommendations..................... 122

$\begin{array}{ll}\text { References } & 125\end{array}$

A ENO Scheme Formulation $\quad 135$

A.1 Introduction . . . . . . . . . . . . . . . 135

A.2 Interpolating Polynomials . . . . . . . . . . . . 136

A.3 Scheme Construction . . . . . . . . . . . . . 137

A.4 Time Integration $\ldots \ldots \ldots \ldots$

A.5 1-D Validation . . . . . . . . . . . . . . . . 139

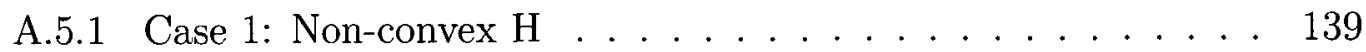

A.5.2 Case 2: Burger's equation (convex H) . . . . . . . . 141

A.6 2-D Validation . . . . . . . . . . . . . . . . 142

A.6.1 Case 3: Non-convex H) . . . . . . . . . . . 142

A.6.2 Case 4: Burger's equation (convex H) . . . . . . . . . 143

B User Information $\quad 144$

B.1 Input Files . . . . . . . . . . . . . . . . 144

B.2 Output Files. . . . . . . . . . . . . . . 145

B.3 Script Files . . . . . . . . . . . . . . . . 146

viii 
B.4 Compiling and Linking . . . . . . . . . . . . . . 146

B.5 Simulation Instructions . . . . . . . . . . . . 146

B.5.1 Geometry Creation .............. 147

B.5.2 Simulation Execution . . . . . . . . . . . . . 149

$\begin{array}{ll}\text { C Code Flow Diagram } & 150\end{array}$

C.1 Main Code Structure . . . . . . . . . . . . . 150

C.2 Detailed Code Structure . . . . . . . . . . . . . . 151 


\section{List of Tables}

2.1 Blob functions and mollified velocity kernels $[r=(x, y)] \ldots \ldots 46$

5.1 HEPM input parameters: STEADY1 . . . . . . . . . . 78

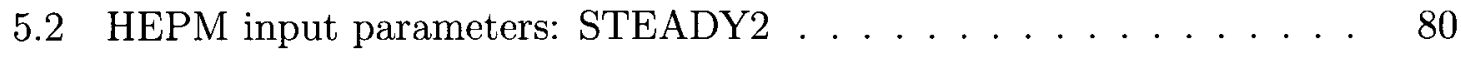

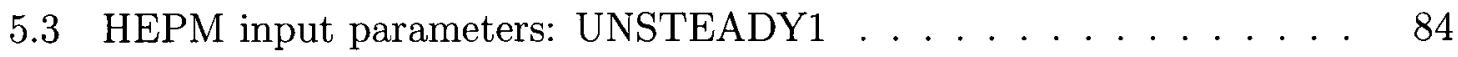

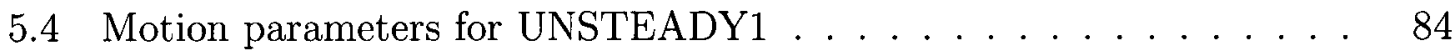

5.5 Euler grid densities and runtimes for vorticity preservation investigation 88

5.6 AGARD experimental specifications . . . . . . . . . . . 96

5.7 HEPM input parameters: UNSTEADY2 . . . . . . . . . . 97

5.8 AGARD CT6 validation case $\ldots \ldots \ldots \ldots \ldots \ldots$

5.9 HEPM input parameters: UNSTEADY3 $\ldots \ldots \ldots \ldots \ldots$

5.10 AGARD CT5 validation case $\ldots \ldots \ldots \ldots \ldots$

5.11 HELINOISE $947 \ldots \ldots \ldots \ldots \ldots \ldots$

5.12 HEPM input parameters: AVI1 . . . . . . . . . . . . . . . . 108

5.13 HEPM input parameters: AVI2 . . . . . . . . . . . . . . 113 
A.1 TVD Runge-Kutta coefficients . . . . . . . . . . . 138

B.1 HEPM Input Files . . . . . . . . . . . . . . . . . 144

B.2 HEPM ........................... 145

B.3 HEPM Script Files . . . . . . . . . . . . . . . 146 


\section{List of Figures}

1.1 Complex rotorcraft aerodynamic environment [2]. $\ldots \ldots \ldots \ldots$

1.2 Illustration of the Smart Hybrid Active Rotor Control System (SHARCS)

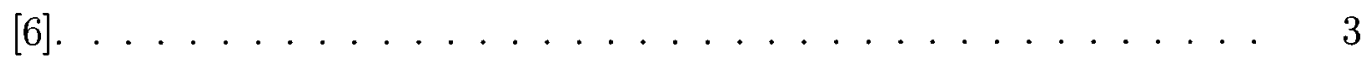

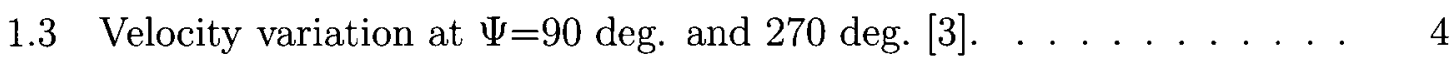

1.4 Hovering helicopter showing the vortical rotor wake through natural condensation of water vapor inside the tip vortex cores $[7] \ldots \ldots 6$

1.5 Laser light sheet flow visualization images of the rotor wake structure during simulated hovering flight. Vortex structures are shown for (a)

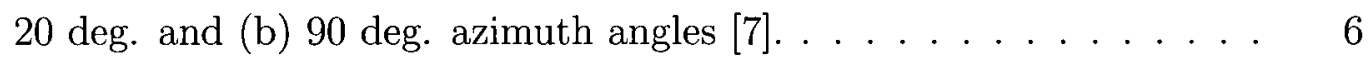

1.6 Top (a) and side (b) views of the blade section in BET [7] $\ldots \ldots .8$

1.7 Prescribed wake model as developed by Landgrebe [9]. $\ldots \ldots \ldots$

1.8 (a) Full helicopter simulation in forward flight with GENUVP [16] and (b) a hover start-up simulation using SMARTROTOR [17] . . . . . . 12

1.9 The formation of vortex particles from a trailing edge wake strip in GENUVP $[18] . \ldots \ldots \ldots \ldots \ldots \ldots \ldots$ 
1.10 (a) DLR CHIMERA grid system and (b) the ONERA sliding mesh system $[23] . \ldots \ldots \ldots \ldots \ldots$

1.11 (a) An example of a vortex particle distribution and (b) the associated non-dimensional vorticity field. . . . . . . . . . . . 18

1.12 2-D block structure for the fast multipole method. . . . . . . . . . 20

1.13 FMM illustration depicting net effect of far-away particles (a) without FMM and (b) with FMM ................ 21

1.14 (a) Representation of overlapping domain hybrid method and (b) isovorticity contours for an overlapping hybrid method of a flow about a cylinder [27]........................ 27

2.1 Velocity kernel distribution for mollified and unmollified kernels $(\varepsilon=0.1) .45$

3.1 Illustration of implementation of pseudo and real time integration in

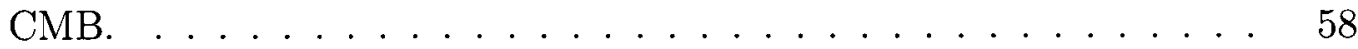

4.1 HEPM model. . . . . . . . . . . . . . . . . . . . . . . . 62

4.2 Illustration of PM to Euler grid compatibility. . . . . . . . . . . . 63

4.3 Cell geometry for the integration of the Euler solution into a particle field. . . . . . . . . . . . . . . . . 67

4.4 Transformation geometry for cell finite-element 2-D linear interpolation. 69 
4.5 Side and top view of the hybrid model including the newly developed non-reflective boundary condition. The top view demonstrates the radial variation of artificial convective velocity and dilatation cut-off

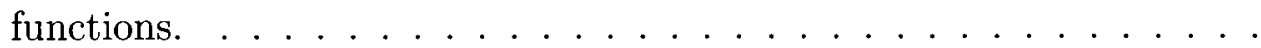

4.6 Time history of particle mesh kinetic energy for a impulsively pitched NACA 0012 airfoil with non-reflective boundary conditions (solid line) and without the non-reflective condition (dashed line). . . . . . .

4.7 Dilatation contours at three timesteps with and without non-reflective boundary conditions implemented. Sampling timesteps are $n=1,11,14$ for a NACA 0012 airfoil in impulsive pitch at $\mathrm{M}=0.5 . . . . . .$. .

4.7 Dilatation contours at three timesteps with and without non-reflective boundary conditions implemented. Sampling timesteps are $n=1,11,14$ for a NACA 0012 airfoil in impulsive pitch at $M=0.5$. (cont) . . . . .

5.1 Density contours at (a) $n=1$ and (b) $n=1000$ timesteps for the STEADY1

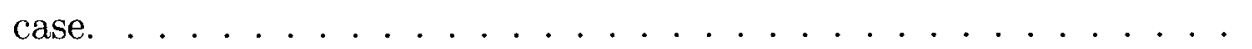

5.2 Dilatation contours at (a) $n=1$ and (b) $n=1000$ timesteps for the STEADY1 case. ....................... 79

5.3 Vorticity contours at (a) $n=1$ and (b) $n=1000$ timesteps for the STEADY1 case. ....................... 80 
5.4 Density contours at (a) $n=1$ and (b) $n=1000$ timesteps for the STEADY2 case. .......................... 81

5.5 Dilatation contours at (a) $n=1$ and (b) $n=1000$ timesteps for the STEADY2 case. ............................ 81

5.6 Vorticity contours at (a) $n=1$ and (b) $n=1000$ timesteps for the STEADY2 case. . . . . . . . . . . . . . . . . . . .

5.7 Chordwise $C_{p}$ distribution for $\mathrm{n}=1$ (solid line) and $\mathrm{n}=1000$ (square) timesteps for (a) STEADY1 and (b) STEADY2 cases . . . . . . .

5.8 Lift hysteresis convergence history for the UNSTEADY1 case. . . . .

5.9 Lift hysteresis comparison between Euler reference solution and HEPM for the UNSTEADY1 case. Results shown for the 3rd cycle. . . . . .

5.10 Lift history comparison between Euler reference solution and HEPM for the UNSTEADY1 case. Results shown for the 3rd cycle. . . . . .

5.11 (a) Vorticity and (b) dilatation contours as they cross the interface (squares) of the overlap between the two methods for the UNSTEADY1

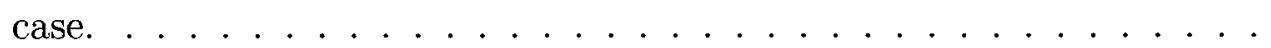

5.12 Downstream region of interest for vorticity preservation investigation with the Euler solver $(\mathrm{CMB}) . \ldots \ldots \ldots$ 
5.13 Euler grids for demonstrating numerical dissipation of vorticity. Grid densities are illustrated for the region of interest identified in Figure 5.12: (a) coarse (b) medium (c) fine (d) extra fine. . . . . . . . . .

5.14 Vorticity contours on the Euler grids for (a) coarse (b) medium (c) fine and $(\mathrm{d})$ extra fine densities. . . . . . . . . . . . . . 91

5.14 Vorticity contours on the Euler grids for (a) coarse (b) medium (c) fine and $(\mathrm{d})$ extra fine densities. (cont) $\ldots \ldots \ldots \ldots$. . . . . . 92

5.15 Downstream region of interest for vorticity preservation investigation with HEPM. . . . . . . . . . . . . . . . . . . . . . . . 9 93

5.16 Vorticity contours in the above region of interest. . . . . . . . 93

5.17 Vorticity distribution at $\mathrm{y}=0$ across the domain of interest for the extra fine Euler grid (solid line) and for HEPM (dashed line). . . . . . . 95

5.18 Comparison of normal force history between experimental AGARD CT6 (square), HEPM UNSTEADY2 (black line) and Euler solution

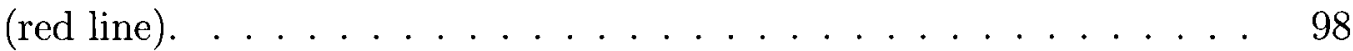

5.19 Pressure coefficient distributions for experimental AGARD CT6, HEPM

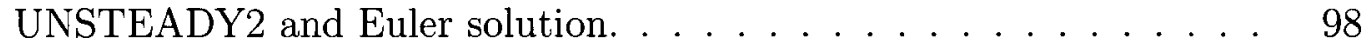

5.19 Pressure coefficient distributions for experimental AGARD CT6, HEPM UNSTEADY2 and Euler solution. (cont) . . . . . . . . . 99

5.20 Lift hysteresis convergence history for HEPM UNSTEADY3. . . . . . 100 
5.21 Comparison of converged normal force over the 3rd cycle for the experimental AGARD CT5 (square), Euler reference (red line) and HEPM UNSTEADY3 (black line) solution. . . . . . . . . . . . . . . . 101

5.22 Comparison of the converged normal force time history over the 3rd cycle for experimental AGARD CT5 (square), Euler reference (red line) and HEPM UNSTEADY3 (black line) solutions. . . . . . . . . . 101

5.23 Pressure coefficient distributions for AGARD CT5 case. . . . . . . . 102

5.23 Pressure coefficient distributions for AGARD CT5 case. (cont) . . . 103

5.24 Core distribution of swirl velocity (a) and associated vorticity contours (b) for the vortex initialized in the AVI studies. . . . . . . 106

5.25 HEPM model for AVI studies. . . . . . . . . . . . . . . . . 107

5.26 Vorticity contours for the AVI1 case $(\mathrm{e}=0.0) \ldots \ldots \ldots \ldots \ldots$

5.27 Lift coefficient history for AVI1 case at various vortex miss distances. 111

5.28 Pressure coefficient history at upper $\mathrm{x} / \mathrm{c}=0.055$ position for AVI1 case at various vortex miss distances. . . . . . . . . . . . . 111

5.29 Pressure coefficient history at lower $\mathrm{x} / \mathrm{c}=0.055$ position for AVI1 case at various vortex miss distances. . . . . . . . . . . . . . 112

5.30 Lift coefficient history for AVI2 case at various vortex miss distances. 114

5.31 Pressure coefficient history at upper $\mathrm{x} / \mathrm{c}=0.055$ position for AVI2 case at various vortex miss distances. . . . . . . . . . . . . . . . . 114 
5.32 Pressure coefficient history at lower $\mathrm{x} / \mathrm{c}=0.055$ position for AVI2 case at various vortex miss distances. . . . . . . . . . . . . . . . 115

A.1 Discontinuity capturing for 1-D non-convex problem using various monotonic fluxes: Lax-Freidrichs (LF), Godunov (GOD), Local Lax-Freidrichs (LLF) and Roe's flux with LLF entropy correction (RF). $\left(t=1.5 / \pi^{2}\right)$

A.2 Comparison of numerical and exact solution to 1-D non-convex problem. Roe's flux with LLF entropy correction implemented. $\left(t=1.5 / \pi^{2}\right) \quad 140$

A.3 Discontinuity capturing for Burger's problem using various monotonic fluxes: Lax-Freidrichs (LF), Godunov (GOD), Local Lax-Freidrichs (LLF) and Roe's flux with LLF entropy correction (RF). $\left(t=1.5 / \pi^{2}\right)$

A.4 Comparison of numerical and exact solution to Burger's problem. Roe's flux with LLF entropy correction implemented. $\left(t=1.5 / \pi^{2}\right) \ldots$. . . . . 142

A.5 Comparison of exact (left) and numerical (right) solutions to the 2D non-convex equation. Numerical solution employs the Roe's scheme with LLF entropy correction. $\left(t=1.5 / \pi^{2}\right) \ldots \ldots . . . . . . .143$

A.6 Comparison of exact (left) and numerical (right) solutions to the 2D Burger's equation. Numerical solution employs the Roe's scheme with

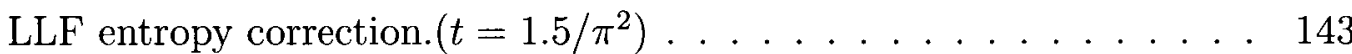

B.1 HEPM directory structure . . . . . . . . . . . . . . 147

B.2 HEPM block numbering scheme . . . . . . . . . . . . . . . 148 xviii 


\section{List of Symbols}

$\begin{array}{ll}c & \text { airfoil chord } \\ C & \text { Hamilton-Jacobi constant } \\ C_{l} & \text { lift coefficient } \\ C_{p} & \text { pressure coefficient } \\ C_{T} & \text { thrust coefficient } \\ d D & \text { blade element drag force } \\ d L & \text { blade element lift force } \\ d M & \text { blade element moment } \\ d y & \text { blade element width } \\ e & \text { specific energy } \\ E & \text { specific total energy } \\ f & \text { right hand side of Hamilton-Jacobi equation }\end{array}$

xix 


$\begin{array}{ll}\vec{F}, \vec{G} & \text { flux vectors } \\ G & \text { Green's function } \\ H & \text { Hamiltonian } \\ i & \text { specific enthalpy } \\ \vec{I} & \text { identity vector } \\ \text { Interp } & \text { Interpolation operator } \\ k & \text { reduced frequency } \\ \vec{K} & \text { Biot-Savart velocity kernel } \\ \vec{K}_{\varepsilon} & \text { mollified Biot-Savart velocity kernel } \\ \text { Proj } & \text { Projection operator } \\ p_{0} & \text { pseudo-time index } \\ M & \end{array}$




\begin{tabular}{|c|c|}
\hline$q$ & pseudo-pressure \\
\hline$r$ & radial distance from vortex core \\
\hline$r_{c}$ & vortex radial core size \\
\hline$R$ & rotor radius \\
\hline $\operatorname{Re}$ & Reynolds' number \\
\hline$\vec{R}_{i, j}$ & flux residual for cell $(\mathrm{i}, \mathrm{j})$ \\
\hline$s$ & entropy \\
\hline$t$ & time \\
\hline$T$ & fluid temperature \\
\hline$\vec{u}$ & fluid velocity \\
\hline$U$ & blade element inflow velocity \\
\hline$U_{c}$ & artificial convection velocity \\
\hline$U_{\max }$ & maximum value of artificial convection velocity \\
\hline$u, v$ & cartesian components of velocity \\
\hline$U, V$ & cartesian components of contravariant velocity \\
\hline$u_{n}, u_{t}$ & normal/tangential disturbance velocity of fluid near the surface \\
\hline$V_{\theta}$ & vortex swirl velocity \\
\hline$\vec{w}$ & solenoidal velocity \\
\hline$\vec{W}$ & vector of conserved variables \\
\hline$\vec{x}_{p}$ & particle position vector \\
\hline
\end{tabular}




\begin{tabular}{|c|c|}
\hline$x_{t}, y_{t}$ & Euler grid velocity cartesian components \\
\hline$\alpha$ & Runge-Kutta time integration coefficient matrix \\
\hline$\alpha_{m}$ & mean incidence (sinusoidal airfoil pitch) \\
\hline$\alpha_{0}$ & pitch amplitude (sinusoidal airfoil pitch) \\
\hline$\beta$ & Runge-Kutta time integration coefficient matrix \\
\hline$\gamma$ & ratio of specific heats $\left(c_{p} / c_{v}\right)$ \\
\hline$\Gamma$ & circulation \\
\hline$\Gamma_{p}$ & particle circulation \\
\hline$\Gamma_{v}$ & Scully vortex strength \\
\hline$\delta$ & dilatation \\
\hline$\delta_{c}$ & Dirac measure \\
\hline$\delta_{p}$ & particle dilatation \\
\hline$\Delta$ & divergence \\
\hline$\Delta_{p}$ & particle divergence \\
\hline$\varepsilon$ & particle cut-off length \\
\hline$\varepsilon_{c}$ & convergence criterion \\
\hline$\eta$ & vertical transformed coordinate \\
\hline$\theta$ & dilatation \\
\hline$\kappa$ & particle overlap ratio \\
\hline$\lambda$ & CFL number \\
\hline
\end{tabular}




\begin{tabular}{ll}
$\Lambda$ & particle-mesh interpolation/projection stencil \\
$\xi$ & horizontal transformed coordinate \\
$\rho$ & fluid density \\
$\rho_{0}$ & fluid reference density \\
$\rho_{p}$ & particle density \\
$\sigma$ & rotor disc solidity ratio \\
$\phi$ & scalar velocity potential \\
$\Phi$ & viscous dissipation \\
$\psi$ & vector velocity potential \\
$\Psi$ & rotor azimuth angle \\
$(\cdots)_{p}$ & particle solution \\
$\omega$ & vorticity \\
$\omega_{p}$ & particle vorticity \\
\hline$)_{p m}$ & rotor angular velocity \\
&
\end{tabular}




\section{List of Abbreviations}

$\begin{array}{ll}\text { AVI } & \text { Airfoil-Vortex Interaction } \\ \text { AGARD } & \text { Advisory Group for Aerospace Research and Development } \\ \text { BET } & \text { Blade Element Theory } \\ \text { BILU } & \text { Block Incomplete Lower Upper } \\ \text { BVI } & \text { Blade-Vortex Interaction } \\ \text { CFD } & \text { Computational Fluid Dynamics } \\ \text { CFL } & \text { Courant-Friedrichs-Levy } \\ \text { CMB } & \text { Carleton Multi-Block } \\ \text { CSD } & \text { Computational Structural Dynamics } \\ \text { CRD } & \text { Collaborative Research and Development } \\ \text { CVPM } & \text { Compressible Vortex Particle Method } \\ \text { DLR } & \begin{array}{l}\text { Deutsches Zentrum für Luft-und Raumfahrt } \\ \text { (German Aerospace Centre) }\end{array}\end{array}$

xxiv 


\begin{tabular}{ll} 
DNW & German-Dutch Wind Tunnel \\
DVPM & Dilating Vortex Particle Method \\
ENO & Essentially Non-Oscillatory \\
FMM & Fast Multipole Method \\
FV & Finite Volume \\
GCG & General Conjugate Gradient \\
GENUVP & General Unsteady Vortex Particle \\
HEPM & Hybrid Euler-Particle Method \\
LES & Large-Eddy Simulation \\
LF & Lax-Friedrichs \\
LLF & Local Lax-Friedrichs \\
MPI & Message-Passing Interface \\
MUSCL & Monotone Upstream-Centered Schemes for Conservation Laws \\
NTUA & National Technical University of Athens \\
NSERC & National Sciences and Engineering Research Council \\
OCE & Ontario Centres of Excellence \\
ODE & Ordinary Differential Equation \\
ONERA & $\begin{array}{l}\text { Office National d'Etudes et de Recherches Aérospatiales } \\
\text { (French Aerospace Laboratory) }\end{array}$ \\
PM & Particle-Mesh \\
\hline
\end{tabular}




$\begin{array}{ll}\text { PSE } & \text { Particle Strength Exchange } \\ \text { RF } & \text { Roe's flux with LLF entropy correction } \\ \text { RRG } & \text { Rotorcraft Research Group } \\ \text { SHARCS } & \text { Smart Hybrid Active Rotor Control System } \\ \text { SRO } & \text { Special Research Opportunity } \\ \text { TVD } & \text { Total Variation Diminishing } \\ \text { VIC } & \text { Vortex-In-Cell } \\ \text { VTOL } & \text { Vertical Take-Off and Landing }\end{array}$

xxvi 


\section{Chapter 1}

\section{Introduction.}

\subsection{Motivation}

Rotorcraft are extremely useful types of aircraft for both commercial and military applications. Their unique hover and vertical take-off and landing (VTOL) capabilities make them well suited to many tasks which could not be accomplished as efficiently by fixed-wing aircraft. An estimated 45,000 rotorcraft are used worldwide for medical transport, law enforcement, fire-fighting, offshore transport, surveillance and attack [1].

Unfortunately, these unique vehicles operate in an unsteady and severe aerodynamic environment. In fact, it has been said that in one rotor blade revolution almost all known aerodynamic phenomena are encountered [2]. These phenomena, demonstrated in Figure 1.1, include such effects as:

- transonic flow on the advancing blades;

- shock-boundary layer interactions; 
- low-speed or even reversed flow on the retreating blades;

- periodic variation of the resultant angle of attack within one revolution leading to dynamic stall on the retreating blades;

- strong 3-D spanwise flow on the blades as they align with the freestream direction;

- the formation of helical tip vortices and their interactions with the other rotor blades, called blade-vortex interaction (BVI).

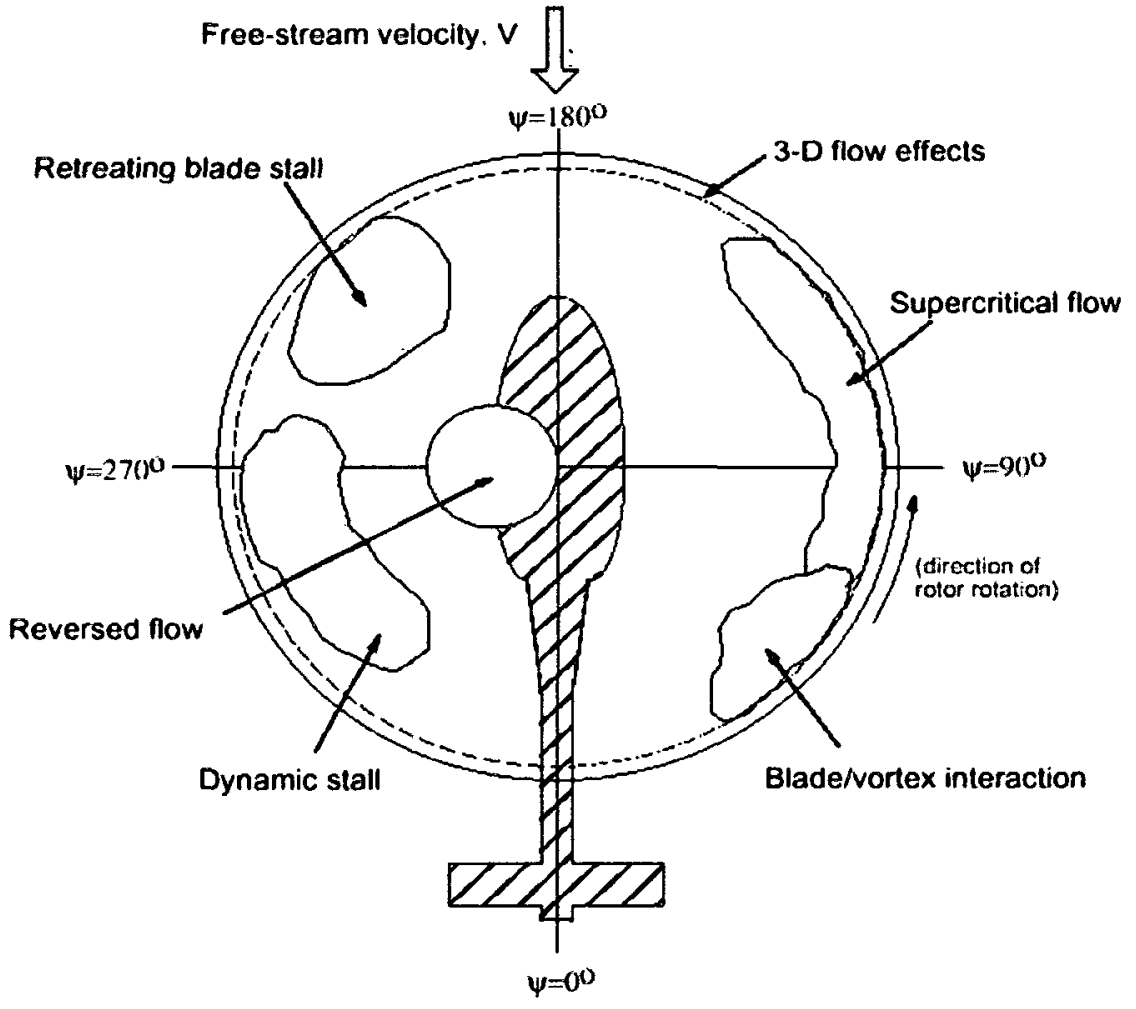

Figure 1.1: Complex rotorcraft aerodynamic environment [2]. 
Due to this severe and highly unsteady aerodynamic environment, rotorcraft are characterized by significant noise and vibration, the reduction of which has become one of the most active research areas in the rotorcraft community. One of the groups focusing on this vibration and noise reduction is the Rotorcraft Research Group (RRG) at Carleton University, which integrates research efforts in the fields of rotorcraft aerodynamics, aeroelasticity, aeroacoustics, blade dynamics and smart structures. The main project of this group is the Smart Hybrid Active Rotor Control System (SHARCS) [3], whose goal is to mitigate noise and vibration on helicopters via a novel hybrid control approach, in which multiple active control systems are considered on one blade [4] [5], Figure 1.2.

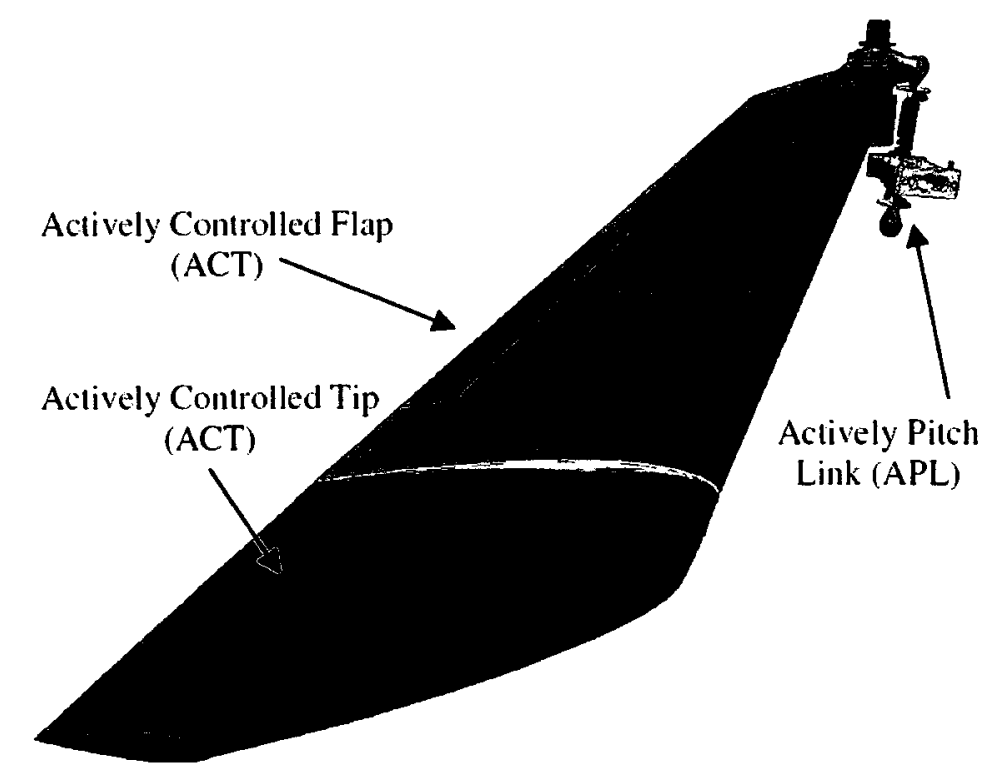

Figure 1.2: Illustration of the Smart Hybrid Active Rotor Control System (SHARCS) [6].

In order to aid the design of the SHARCS system, it is necessary to perform 
accurate rotor and hub air load predictions, as well as to simulate the complete flowfield via computational fluid dynamics (CFD). However, because of the need to model blade flapping, lead-lag and torsional motions, a rotorcraft CFD method also has to be coupled with a Computational Structural Dynamics (CSD) model. Development of such 3-D comprehensive rotorcraft aeroelastic codes is currently a matter of research worldwide. Although there have been various methods developed and tested recently (a complete overview will be given in the following sections), an area particularly lacking is a CFD model capable of capturing both near-blade and far-field aerodynamic effects accurately at the same time.

As shown in Figure 1.3, the forward flight of a rotorcraft causes an asymmetric velocity distribution between the advancing and retreating blades, resulting in numerous near-blade phenomena.

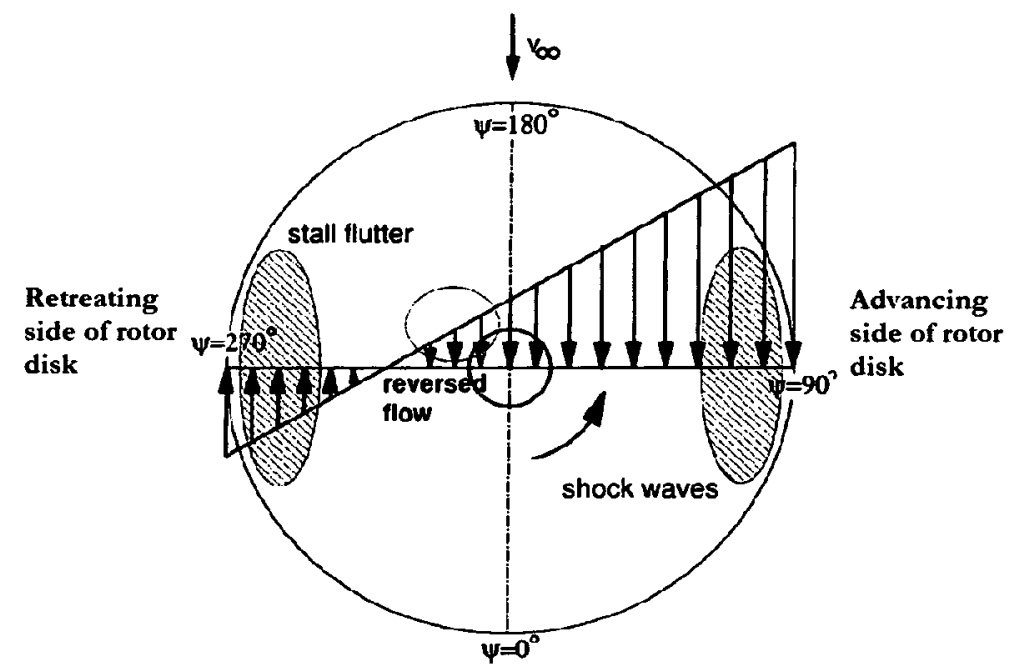

Figure 1.3: Velocity variation at $\Psi=90 \mathrm{deg}$, and $270 \mathrm{deg}$. [3]. 
On the advancing side, there is transonic flow causing shock-waves to form which subsequently interact with the boundary layer. On the retreating side, dynamic stall conditions are originating from boundary layer separation either at the leading edge or trailing edge of the blades. These conditions are also affected by boundary layer transition from laminar to turbulent and separation bubbles. In addition, it is important to resolve flow reattachment points in order to properly capture lift, moment and drag hysteresis. Therefore, capturing the near-blade aerodynamics is absolutely crucial for accurately modeling rotorcraft flows.

However, for simulating rotorcraft vibration and noise it is essential to capture the far-field phenomena as well. This concerns the existence of a wake vortex structure, which is essentially the vortices shed from the rotor blade tips in a helical pattern. This will impact or otherwise interact with the following blades which is known as blade-vortex interaction (BVI). The helical wake structure is demonstrated in Figures 1.4 and 1.5 . 


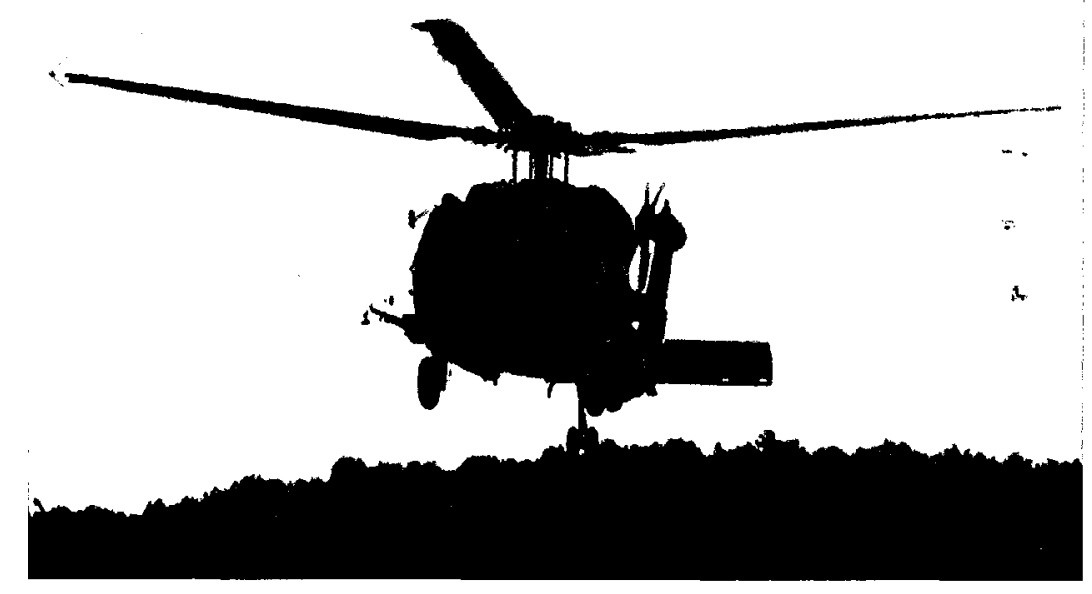

Figure 1.4: Hovering helicopter showing the vortical rotor wake through natural condensation of water vapor inside the tip vortex cores [7].

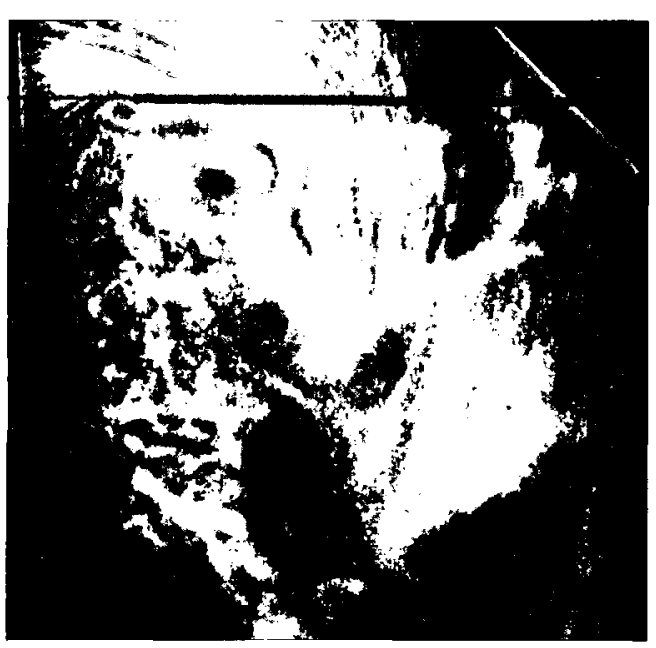

(a)

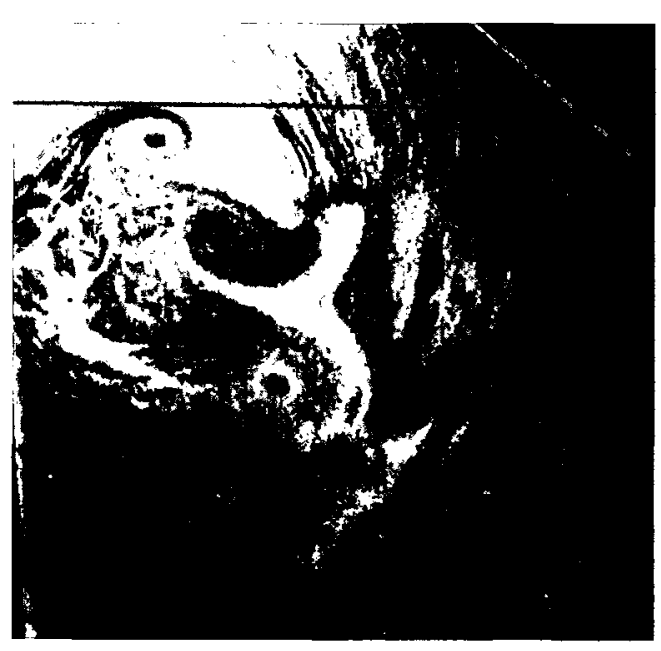

(b)

Figure 1.5: Laser light sheet flow visualization images of the rotor wake structure during simulated hovering flight. Vortex structures are shown for (a) 20 deg. and (b) 90 deg. azimuth angles [7]. 
It is very important that a rotorcraft CFD method be able to resolve the unsteady loading caused by these BVI occurrences, in addition to the complex near-blade phenomena. The next section details the history of rotorcraft aerodynamic modeling and the capabilities and limitations of the various methods.

\subsection{History of Rotorcraft Aerodynamic Modeling}

There has been a wide variety of methods developed for predicting the aerodynamics of rotorcraft, ranging from the analytical blade element theory to various CFD methods. The following literature review discusses the evolution of these methods as well as the limits of their capabilities.

\subsubsection{Blade-Element Theory}

Although its development began in the early 20th century by Polish aviation pioneer Drzewiecke [8], blade element theory (BET) is still used today as a first order analysis in initial rotor design phases. BET provides estimates of radial and azimuthal distributions of the blade aerodynamic loading by assuming that each blade section acts as a quasi-two-dimensional airfoil. By assuming independent blade sections of width $d y$, and an inflow velocity, $U$, it is possible to use two-dimensional airfoil characteristics to determine local forces, $d L, d D$, and moments, $d M$, as shown in Figure 1.6. Rotor performance characteristics can then be determined by integrating sectional loads and moments over the length of the blade and averaging the result over a rotor 
revolution. Note that while the assumption of independent, quasi-2D blade sections is quite acceptable for axisymmetric flowfields (i.e. hover, climb and descent), it is an inaccurate assumption for forward flight, where strong spanwise flow occurs and where vibration and noise is the heaviest.
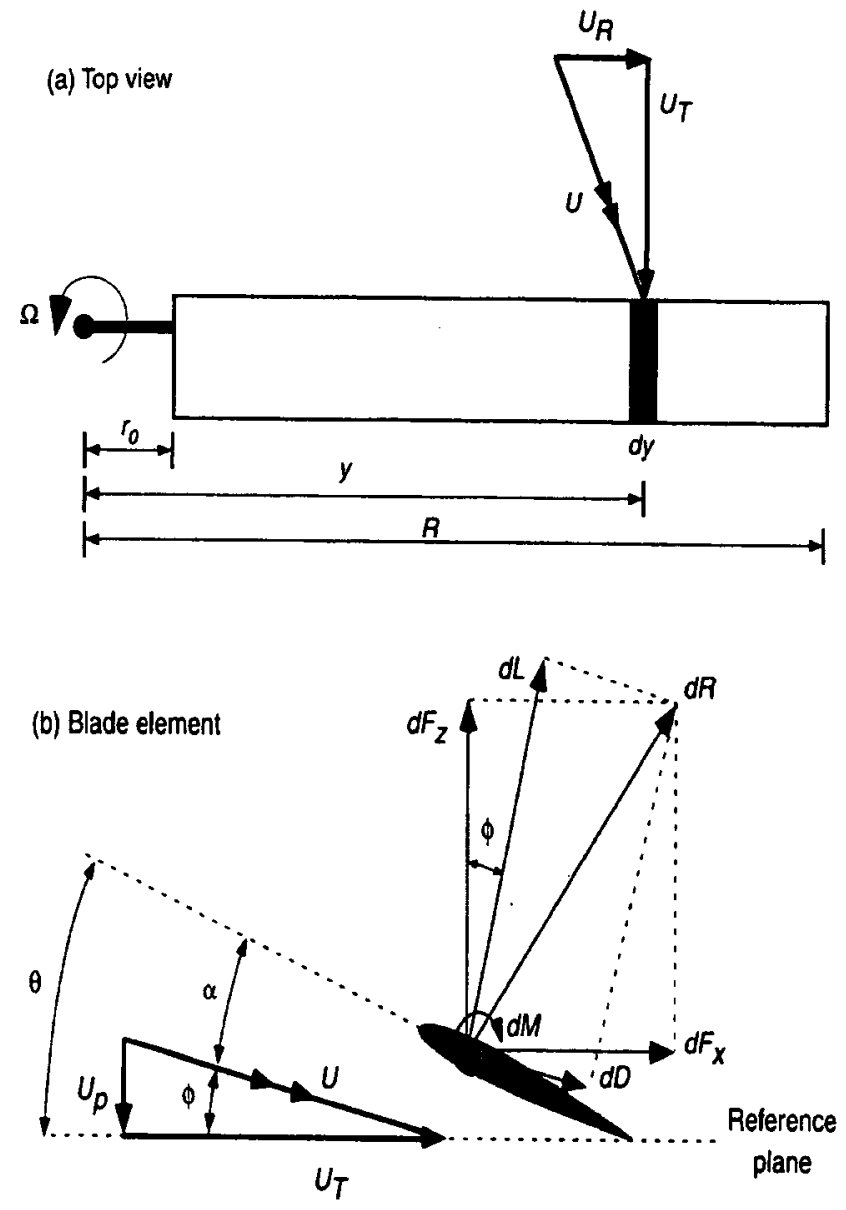

Figure 1.6: Top (a) and side (b) views of the blade section in BET [7].

A key element of BET is the prediction of the inflow distribution over the rotor disc. While this can be modeled fairly accurately for hover and vertical flight, models 
for forward flight are usually very inaccurate and heavily dependent on user adjusted empirical constants. Thus, BET is not sufficient for detailed rotor design work as it is also unable to capture BVI, three-dimensional flow effects and wake vortices [7].

\subsubsection{Prescribed Wake Models}

Prescribed wake models represent methods with more sophisticated rotor inflow models. They are still fairly simple and computationally efficient methods, but without the expense and uncertainties associated with the explicit calculation of the free positions of the wake, as well as the longitudinal and lateral distortions during wake roll-up. The first such model was developed for hover by Landgrebe [9], who studied around seventy rotor configurations with different numbers of blades, rotor solidity, twist and aspect ratio. Based on experimental data, Landgrebe then determined empirical tip vortex geometry equations with tuned constants according to the configuration of interest to determine axial settling rates of the tip vortex. Figure 1.7 demonstrates the Landgrebe model. Kocurek and Tangler [10] then extended this approach with more advanced tuning of the constants to consider blade lift distribution as well.

The prescribed wake methods have been extended to forward flight applications by Coleman et al [11]. This simple model approximates trailed vorticity from the rotor through a series of stacked vortex rings. The advantage of such a model is that an exact analytic solution may be obtained for the induced velocity. In each revolution, there is one ring ejected from the rotor plane, with successive revolutions 


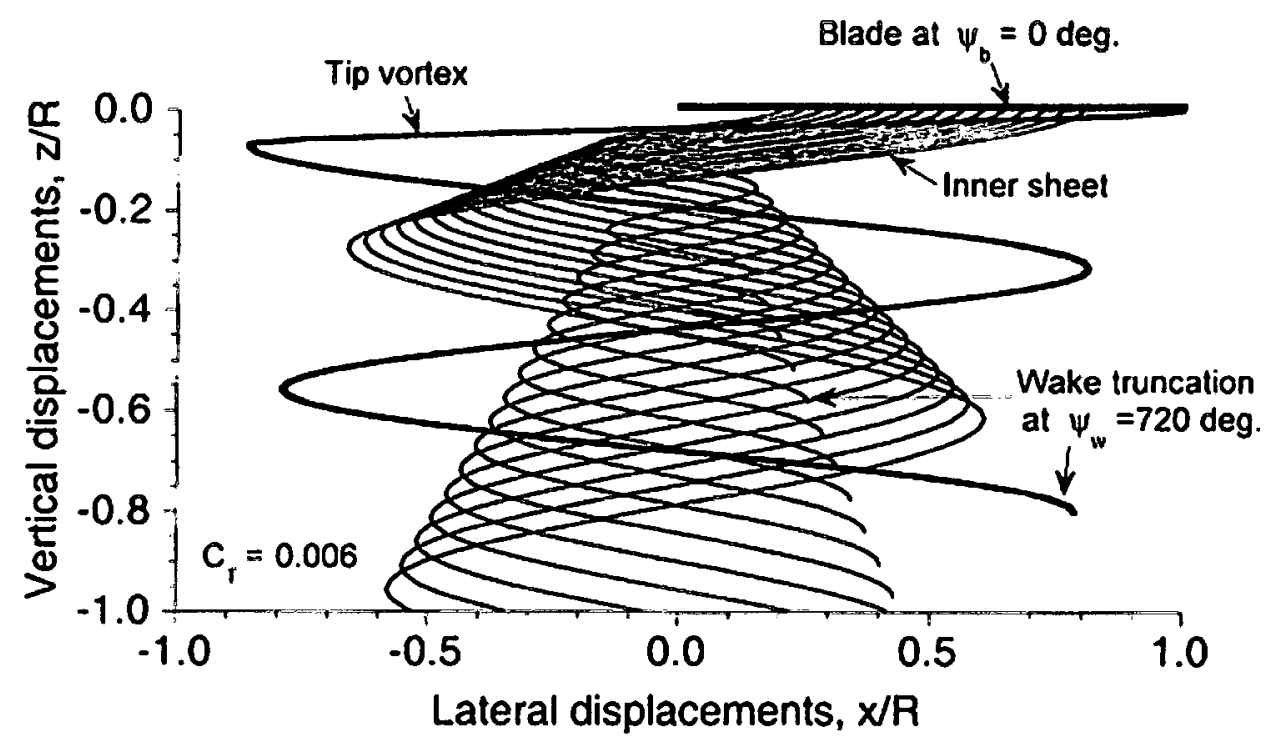

Figure 1.7: Prescribed wake model as developed by Landgrebe [9].

stacking additional rings onto the main stack. The new ring positions are defined on the basis of momentum theory. Note, however, that in reality the wake is a helical tip vortex rather than a set of vortex rings. So there is an obvious discrepancy between this model and the actual flow physics.

Finally, prescribed rigid wake models were updated to include distortions due to wake roll-up by Beddoes [12], with empirical weighting constants being used to provide the distortions. It was found in [12] that the Beddoes model agreed with the overall wake trends seen in experiment, but high resolution of the rotor wake details was not achieved. 


\subsubsection{Free Wake Methods}

In order to capture the rotor wake geometry more accurately and to capture possible BVI effects, a more elaborate description is required for the wake and for the induced velocity distribution over the rotor. This led to the development of free wake methods, which attempt to model the rotor wake evolution according to a set of physics based governing equations. Although the computational costs increase by at least two orders of magnitude in comparison to the prescribed wake models, the free wake method is more accurate in resolving wake details.

In a study done by Lee [13], a free wake analysis was coupled with a threedimensional thin lifting surface representation of the rotor blade. This surface trails bound circulation into the wake. Radial variation of the bound circulation produces trailed vorticity, while azimuthal variation of the bound vorticity produces shed vorticity into the wake. The strength of the trailed and shed vorticity is determined by the radial and azimuthal derivatives of the bound circulation at the time the wake element leaves the blade surface. Once off the surface, the free vortices are convected according to the free-stream velocity and the induced velocities from the thin blade and other wake elements deduced from the Biot-Savart Law. Although good overall results are reported with this method, the lack of viscosity and compressibility in the model limits its validity.

As a free wake description, vortex methods have been implemented in the field 
of rotorcraft CFD. One example of a comprehensive rotorcraft code which takes advantage of the grid-free nature of vortex methods has been developed at the $\mathrm{Na}$ tional Technical University of Athens (NTUA) called the General Unsteady Vortex Particle code (GENUVP) by Voutsinas et al [14-16]. GENUVP is currently used as the aerodynamic component by the Rotorcraft Research Group (RRG) at Carleton University for their in-house rotorcraft aeromechanical software called SMARTROTOR [17]. Figure 1.8 depicts some calculated particle fields for full helicopter and blade-only simulations using GENUVP and SMARTROTOR.

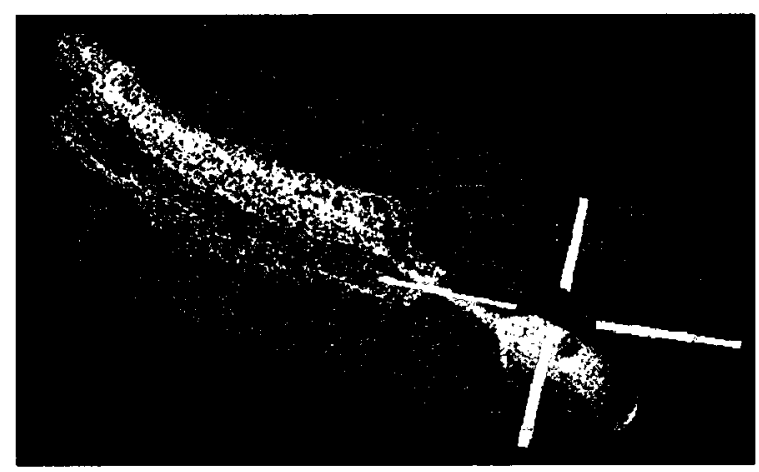

(a)

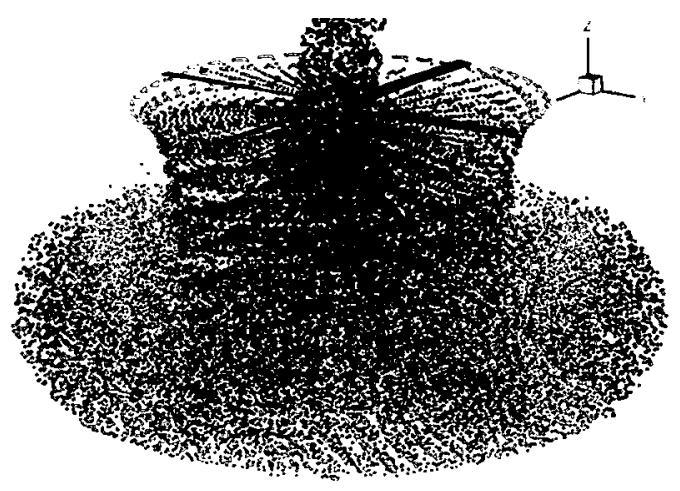

(b)

Figure 1.8: (a) Full helicopter simulation in forward flight with GENUVP [16] and (b) a hover start-up simulation using SMARTROTOR [17].

This unique code couples an indirect panel method with a vortex particle description of the wake. At each timestep, a near-wake strip of panels is convected from the trailing edge of the blade. At the end of the timestep, the dipole intensities on this 
near-wake strip are integrated to form vortex particles. These particles join the particle field and are convected and deformed according to the governing vortex transport equation cast in a Lagrangian sense. This coupling process is demonstrated in Figure 1.9.
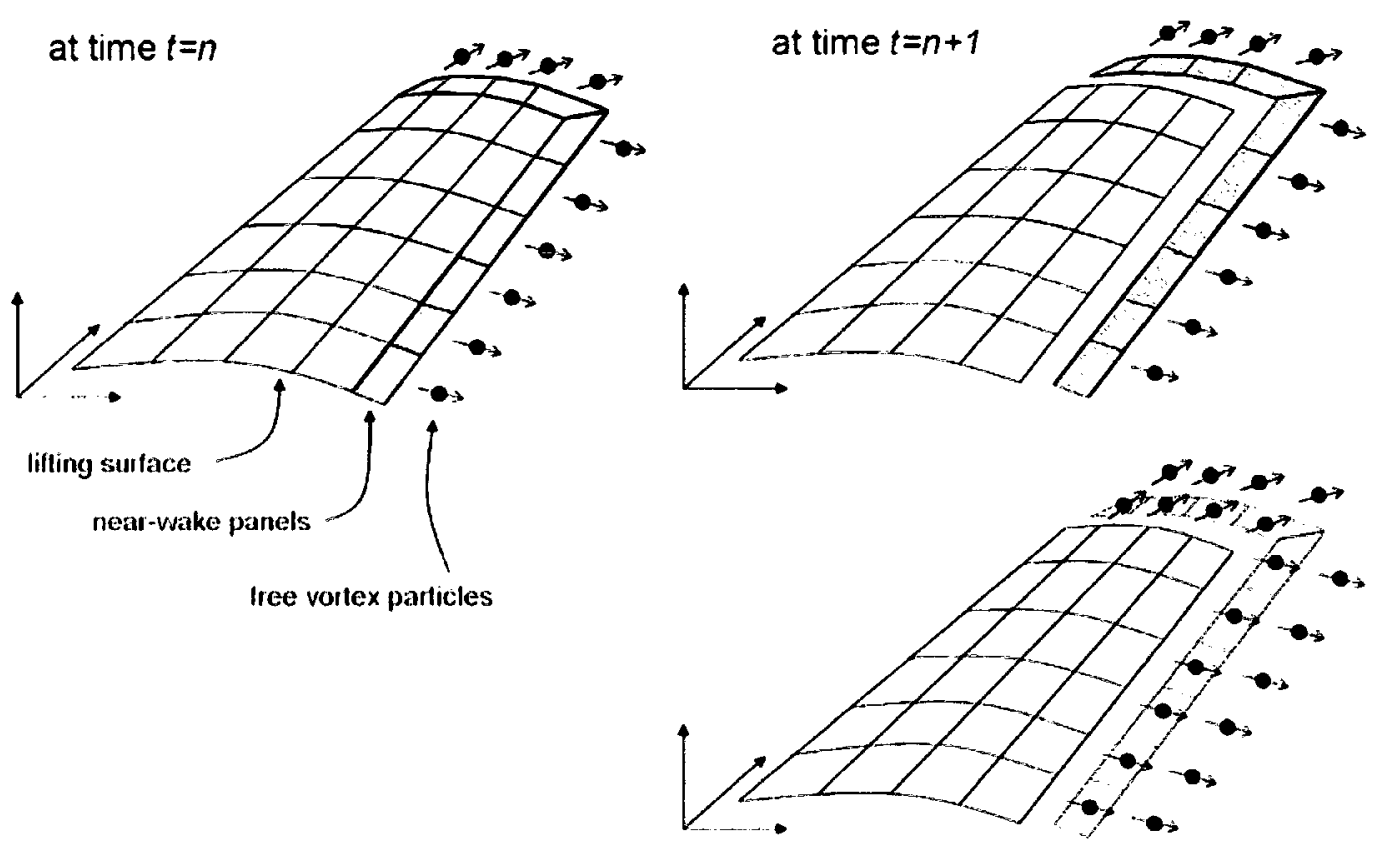

Figure 1.9: The formation of vortex particles from a trailing edge wake strip in GENUVP [18].

The conservative nature of the vortex method disallows the numerical dissipation of vorticity and hence makes them superior for capturing the helical tip vortex wake for rotorcraft simulations. Regarding computational costs, the implementation of fast summation methods for resolving the velocity field can provide an efficient and 
practically feasible method for solving rotorcraft flowfields. However, a vortex particle method is still incompressible in nature, causing limitations in predicting phenomena for the advancing blade, where the tip speed can reach Mach 0.85. Also, vortex particle methods have difficulty resolving solid wall boundary conditions at the rotor blades.

To solve these problems, Berkman et al. [19] and Moulton et al. [20] coupled free wake models and conventional grid-based Navier-Stokes solvers in separate studies. This is done by dividing the flow into three regions made up of a) a small viscous region surrounding the rotor blades, in which the Navier-Stokes equations are solved, b) a potential flow region, which carries the acoustic and pressure waves generated by the rotor to the far field, and c) a Lagrangian scheme for capturing the vorticity leaving the viscous region and convecting into the far field. Although results were not accurate, the relatively low computer runtimes due to the small Navier-Stokes region were noticed and it was suggested that better results would be obtained through higher-order coupling schemes.

\subsubsection{Euler/Navier-Stokes CFD}

More recent advances in rotorcraft aerodynamic modeling feature grid-based techniques, in which the Euler or Navier-Stokes equations are solved over the entire flow domain. For full rotorcraft simulations featuring a main rotor, fuselage and perhaps a tailrotor, it is necessary to implement rotating and non-rotating computational zones. 
The first option is to implement a CHIMERA grid system, used in separate studies by Dietz et al [21], Mamou and Khalid [22] and at DLR (German Aerospace Centre) [23]. The CHIMERA grid system decomposes the complex rotorcraft geometry into a series of geometrically simple overlapping grids. Boundary information is exchanged between the grids via interpolation of the flow variables. The DLR CHIMERA system is shown in Figure 1.10a, where a set of three near-field grids is embedded into a cylindrical background grid for a three-bladed model rotor. Two layers of auxiliary cells are used to store the neighbouring flow values in order to match the solution across the inner blade cuts.

Another option to resolve the governing equations over the moving blades is to implement the sliding grid technique. This is the approach taken by Nam et al [24], Steigl and Barakos [25], and ONERA (French Aerospace Laboratory) [23]. Unlike the CHIMERA method, where the separate grids may occupy the same volume, the sliding grid technique features a sliding surface which provides the boundary between the CFD mesh around stationary components and the rotor-fixed CFD mesh which rotates to account for the movement of the rotor. It is not required that the nodes of two meshes in contact align, as the boundary values may be obtained through interpolation. Figure $1.10 \mathrm{~b}$ illustrates the ONERA grid system featuring a multiblock structured grid enclosing the whole multi-bladed rotor, where each block is generated around a single blade with a $\mathrm{C}-\mathrm{H}$ cylindrical topology. This method also 
imposes an artificial hub boundary which is essentially an inner cylinder limit used to close the grid at the axis of rotation.

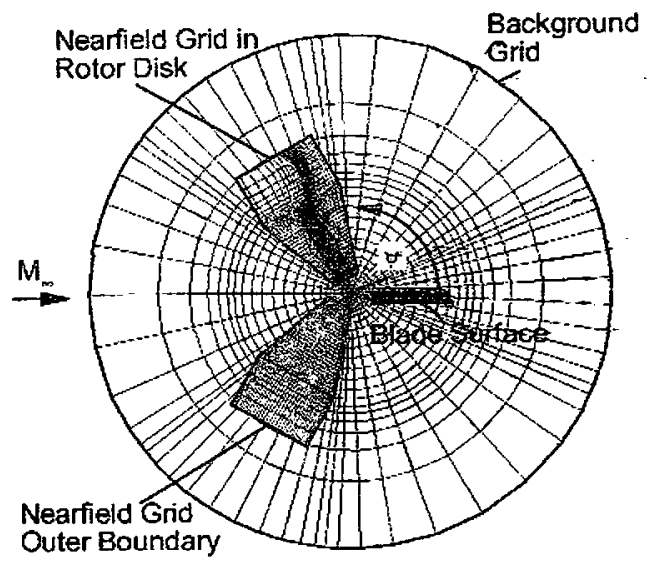

(a)

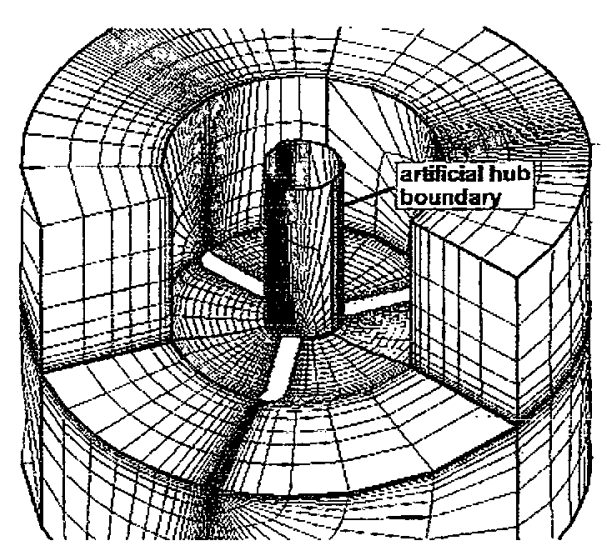

(b)

Figure 1.10: (a) DLR CHIMERA grid system and (b) the ONERA sliding mesh system [23].

Although an attractive approach due to the high resolution of fluid details of these complex fully gridded methods, the mathematical complications associated with matching the rotating grids with the non-rotating ones are of concern and have been identified explicitly by the authors [23]. These methods, just like any conventional grid-based method, are also subject to numerical diffusion of vorticity, caused by the numerical discretization of the convective terms in the governing equations. This causes the unphysical dissipation of the vortex wake structure, thus underpredicting BVI effects. However, a grid-free and conservative Lagrangian vortex particle method does not exhibit these problems, as will be seen in the following sections. 


\subsection{Vortex particle methods}

Vortex particle methods are a subset of vortex methods which discretize the flowfield into a series of markers, (or particles) where local circulation is concentrated. These particles are then convected and deformed according to vorticity evolution and transport equations cast in Lagrangian form. Barba et al [26] discuss the unique advantages of the Lagrangian form of the governing equations. In this form the fluid is viewed as a collection of fluid elements, which are freely translating, rotating and deforming, with individual dependent variables (vorticity,velocity,temperature, etc.) associated with each individual element. The trajectories of these particles are then tracked and a quantity of interest can be sampled at every location in space and instant in time. Thus, the non-linear term of the Euler/Navier-Stokes equations is translated into a system of ordinary differential equation (ODEs) for these trajectories and there is no numerical diffusive truncation error. As opposed to grid-based CFD methods, there is no need for connecting the computational elements, making this method ideally suited to complex geometries with independently moving bodies.

The basic mathematical formulation of the vortex particle method, as can be found in [27], is the following. Particles located at a position $\vec{X}_{p}$ are convecting according to the local fluid velocity:

$$
\frac{D \vec{X}_{p}}{D t}=\vec{V}_{p}
$$


For the simplest case of a 2-D inviscid and incompressible flow, the particle vorticity remains constant in time:

$$
\frac{D \vec{\omega}}{D t}=0
$$

In a 3-D formulation, the term allowing for the tilting and stretching of vorticity must be included:

$$
\frac{D \vec{\omega}}{D t}=\vec{\omega} \cdot(\nabla \vec{V})
$$

Figure 1.11 demonstrates a sample particle distribution along with the associated vorticity field. It is important to note the adaptive nature of the method, with vortex particles concentrating in the areas of highest gradients, lending further advantages to the method.

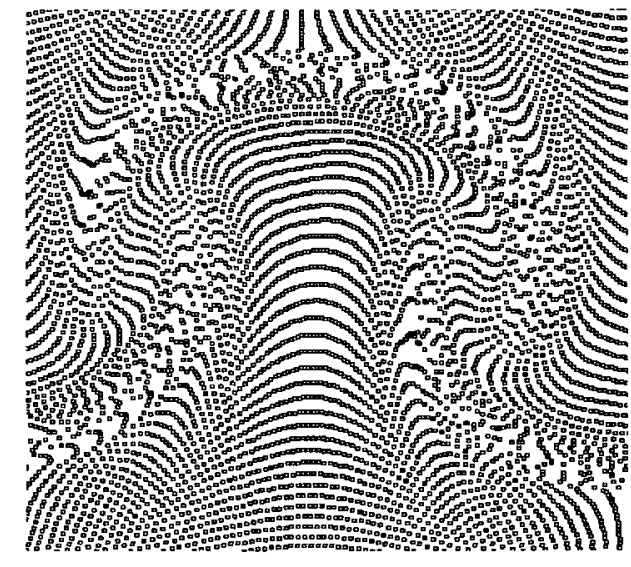

(a)

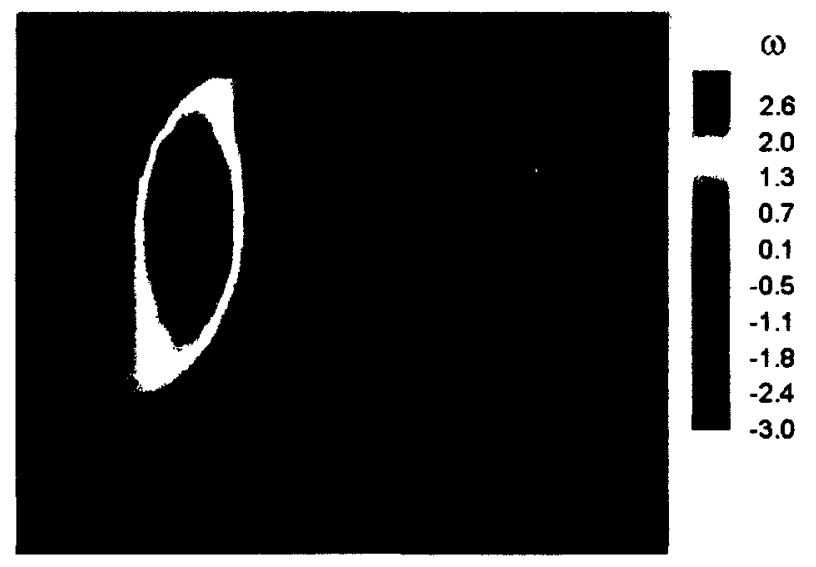

(b)

Figure 1.11: (a) An example of a vortex particle distribution and (b) the associated non-dimensional vorticity field. 
In the past, classical inviscid vortex methods enjoyed the advantages that particles are created only in areas where the vorticity field is non-zero, the automatic adaptivity of the particles (i.e. concentrating into areas of higher vorticity), and the rigorous treatment of the boundary conditions at infinity. Despite this, vortex particle methods have been quite unpopular in the past because of high computational costs, the difficulty of modeling viscosity and the lack of compressibility considerations due to the omission of the irrotational component in the decomposition of the velocity field. Advancements in these areas are discussed in the following sections.

\subsubsection{Fast Summation Techniques}

The issue of high computational demands has been addressed by fast multipole methods (FMM) developed by Greengard and Rokhlin [28] and also by the particle-mesh technique. The FMM has been called one of the top ten algorithms developed in the 20 th century [29]. It addresses a wide range of practical problems because systems are frequently found in nature in which pairwise interactions must be solved for a large number of entities. The concept of the method is illustrated in Figure 1.12. Here, a given particle field is subdivided into rectangular domains, which are again subdivided themselves until only a specified number of minimum particles are contained in each box. At this point, a so-called quad-tree diagram is created, which associates the box structure and determines whether a particle point can be considered near or far from the point of evaluation. 


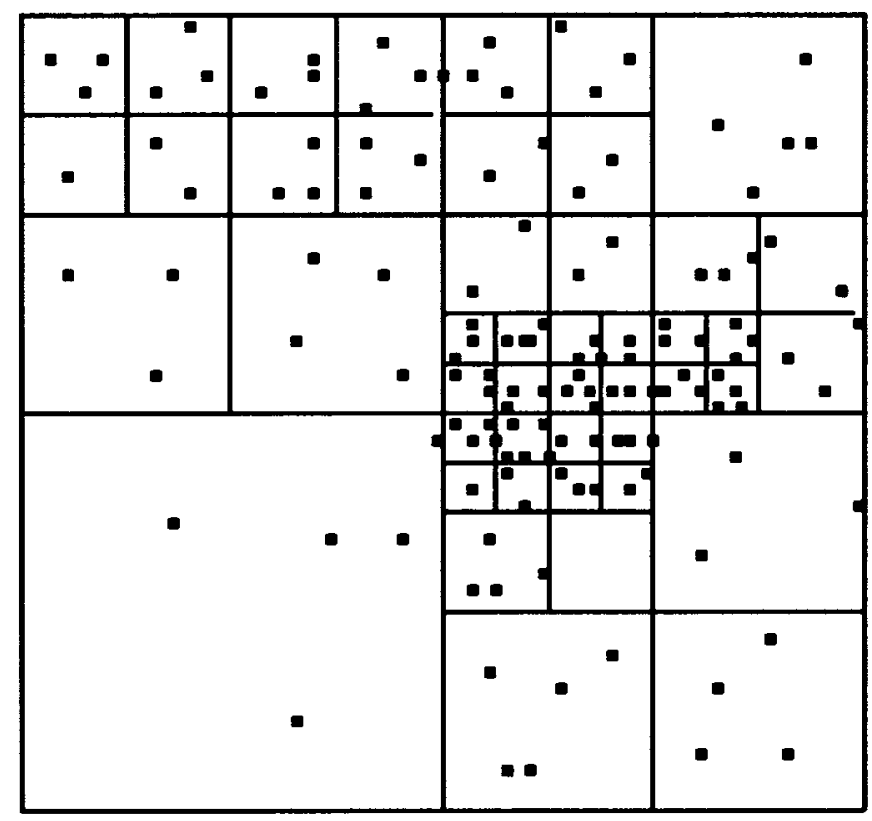

Figure 1.12: 2-D block structure for the fast multipole method.

The idea behind FMM is to essentially take the far-field effect of several vortex particles and approximate them as a single function capturing their net effect, rather than calculating each particle's contribution individually. This process is depicted in Figure 1.13 and essentially reduces the number of calculations required at each timestep from $O\left(N^{2}\right)$ to $O(N)$ or $O(N \log N)$, where $N$ is the number of particles.

The second approach for improving the efficiency of particle methods is the socalled particle-mesh technique. This method is explained in detail by Huberson and Voutsinas [30]. In this approach, the particle vorticity is projected onto a Cartesian mesh containing all of the particles. Once vorticity is known on the mesh nodes, a 


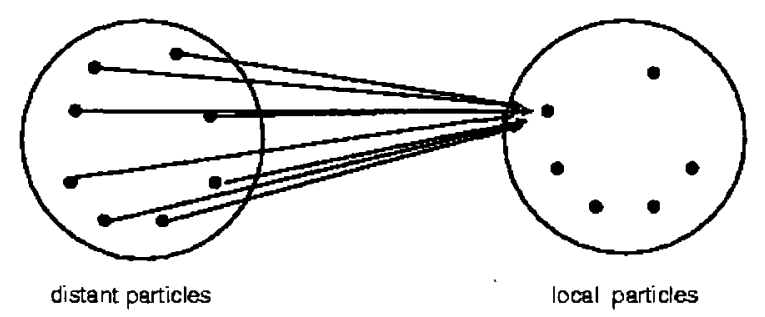

(a)

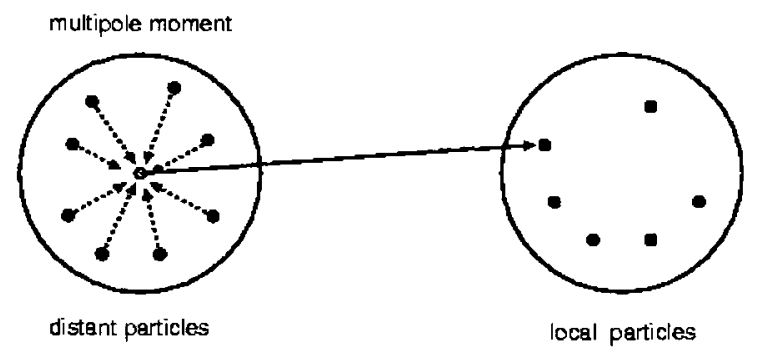

(b)

Figure 1.13: FMM illustration depicting net effect of far-away particles (a) without FMM and (b) with FMM

Fast Fourier Transform (FFT) may be implemented to quickly solve for the mesh velocities. These velocities can then be interpolated back to the particles and they can subsequently be convected, avoiding the direct Biot-Savart velocity convolution. With the particle-mesh approach the computational cost is reduced from $O\left(N^{2}\right)$ to $O(N \log N)$

Although both methods improve the computational efficiency by the same order of magnitude, the FMM is considerably more difficult to implement as it requires the 
creation of a quad-tree data structure in the computer code. As such, the particlemesh technique is implemented as part of this work and will be explained in more detail in Chapter 2.

\subsubsection{Viscous effects}

Vortex particle methods are inherently inviscid, however, viscous models can be included through either random walk, core spreading or particle strength exchange methods. The random walk method was developed by Chorin [31], where the vortex particles and their strengths are respectively convected and diffused in separate steps. At the convection step, the fluid elements are advanced with the local flow velocity, while at the diffusion step these convected elements are then adjusted to modify the vorticity field of the flow. In this step the particles are put through a Brownian type motion based on the probabilistic interpretation of the diffusion equation. Although this method retains the Lagrangian and grid-free nature of vortex methods, it is only effective at modeling slightly viscous flows [32]. The method also has a very low convergence rate and an extremely large density of particles is required for obtaining point-wise accuracy for the computed velocity [27].

Another approach to simulate viscosity is to allow the vortex particles to grow in time with the core spreading technique [33]. Although Greengard [34] showed that this method was not convergent due to inconsistencies with advection without the deformation of larger and larger particles, a viscous-splitting [35] scheme was 
demonstrated to alleviate this issue.

The most common viscous model is the particle strength exchange (PSE) scheme outlined in [36]. In this method, the vorticity is spread between neighboring particles to simulate viscous diffusion. This is done through an integral approximation of the diffusion equation which continuously acts on the vorticity field while it is transported along the trajectories of the fluid elements.

It is not clear which viscous model is most effectively implemented in vortex particle methods. All of the models are extremely dependent on a regularized particle field, with sufficient particle overlap. To ensure this regularity, particle remeshing schemes have been developed but they suffer from numerically diffusive interpolation errors. For this reason, much of the current work in viscous particle methods is focused on location processing and interpolation techniques [32].

\subsubsection{Compressibility effects}

Until recently, one of the major drawbacks of vortex methods was the lack of compressible models. In inviscid vortex methods, it is only necessary to track particles contained in the regions supporting vorticity. However with all compressible approaches it is necessary to track density, for which mass continuity needs to be ensured throughout the entire computational domain. Although this is seen as a disadvantage, the fast computational strategies outlined above allow us to obtain results at acceptable cost and quality for engineering purposes. Currently known compressible 
particle methods are limited to only a few approaches, including the particle-mesh based approaches by Meske [37] and Mas-Gallic [38], as well as the pure-particle approach by Eldredge [39]. The formulations of Meske and Mas-Gallic are particle-mesh style approaches, in which vorticity is tracked along particle paths, while compressibility is calculated on an overlying Cartesian mesh. On the other hand, the approach of Eldredge is a pure-particle method, in which particles themselves carry vorticity, density, dilatation, entropy and enthalpy and all governing equations are solved for in the Lagrangian form.

Unfortunately, the approaches of Meske and Eldredge are applicable to limited cases, while Mas-Gallic's approach was only validated for dilating non-rotational flows. As will be identified in the thesis motivation section, the development of a viable compressible formulation for vortex particle methods will be a major part of the current work. As such, the formulation, advantages and disadvantages of these approaches will be further expanded on in Chapter 2.

It is clear from the discussion above, that vortex particle methods offer a unique solution to rotorcraft CFD issues. Although they lack suitable compressibility models, fast computational methods and viscous models have furthered their applicability. The usefulness of vortex particle methods is particularly suited to the flow domain associated with vortex convection, which leads us to the discussion of hybrid methods. 


\subsection{Hybrid methods}

For CFD analysis, it is desirable to use a method which is representative of the flow physics at hand. In a given simulation, it can be expected that the dominant physics of the flow varies from region to region. In this type of situation, it is advantageous to implement hybrid numerical schemes.

In the case of rotorcraft $\mathrm{CFD}$, it is apparent that a hybrid numerical scheme would be very suitable. A conventional grid-based method could be used to resolve the viscous flow around the blade, while a Lagrangian method would be advantageous to resolve the far-field wake region dominated by convection. By this, boundary layer separation associated with dynamic stall and shock-boundary layer interaction would be resolved by the grid-based method, while the all important vortex wake would be captured accurately and at a low computational cost by the Lagrangian vortex particle method. There are two general classes of hybrid methods involving vortex particle methods: the vortex-in-cell (VIC) method and the particle-grid decomposition method.

Ould-Salihi et al. [40] describe in detail the VIC method. In this approach, the Lagrangian vortex method and an Eulerian grid based method are used in the same flow domains but are applied to different aspects of the flow equations. The vortex method solves the transport equations, while the grid-based method is used to compute the velocity. The diffusion and vorticity boundary conditions can be solved by either 
method. It was found that the VIC method produces excellent high resolution results at high Reynolds' number at a fraction of the time of grid-based finite-difference schemes. However, the need for a fully gridded domain makes this approach impractical for rotorcraft CFD, typically involving several individually moving bodies.

In particle-grid domain decomposition, the Lagrangian vortex method and the Eulerian grid-based method are used to resolve separate parts of the flow domain. The two possible particle-grid decomposition options are an overlapping strategy, as described in [27], or a domain matching strategy described in [41]. Matching domain decomposition involves no overlap between subdomains and hence strict geometrical constraints are imposed on each of them [27]. This causes many complications and convergence issues and therefore indicates that the overlapping domain decomposition, as shown in Figure 1.14, is the most viable hybrid method strategy. In this approach, the Euler/Navier-Stokes gridded domain is defined as V1, the grid-free Lagrangian vortex method domain as V2, while the overlap between the two domains as V12. The coupling mechanism for the overlapping strategy can be summarized as follows:

1. Differentiate velocity grid values to obtain vorticity in $\mathrm{V} 1$;

2. Interpolate vorticity grid values onto particles in V12;

3. Update particle location and circulations in V2 with a particle strength-exchange (PSE) scheme; 
4. Iterate between the two methods until convergence is achieved;

Figure 1.14 shows the capabilities of the overlapping hybrid method. It clearly demonstrates the continuity between the domains of the isovorticity contours which is crucial when combining two different types of numerical methods.

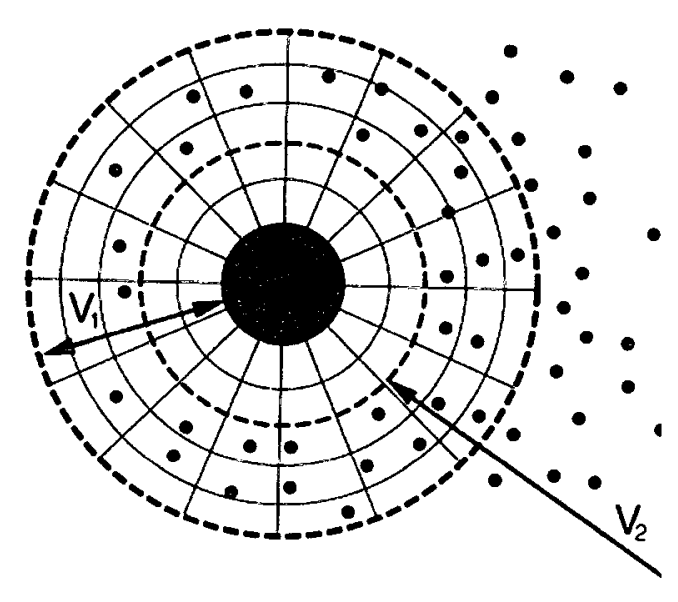

(a)
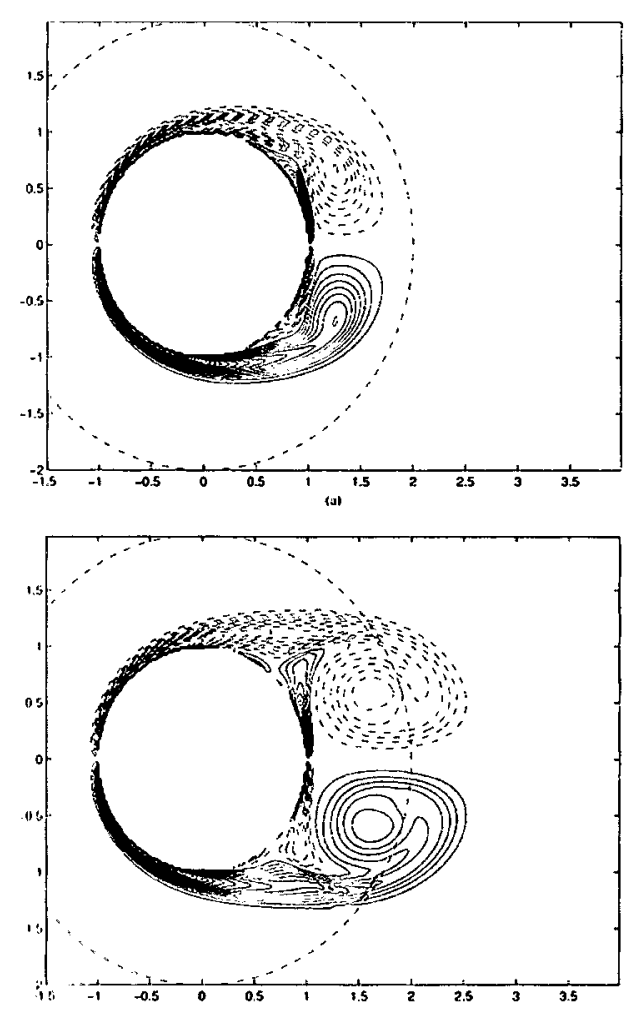

(b)

Figure 1.14: (a) Representation of overlapping domain hybrid method and (b) isovorticity contours for an overlapping hybrid method of a flow about a cylinder [27]. 


\subsection{Thesis Objectives}

It is evident from the preceding literature survey that the prediction of rotorcraft aerodynamics implies major difficulties for CFD. In order to design advanced systems for vibration and noise control, it is necessary to capture the rotorcraft aerodynamic environment accurately. Conventional grid-based Euler/Navier-Stokes methods provide good resolution of the flow details near the blade, but suffer from high numerical dissipation of the vortex wake, require complex meshing strategies and are computationally expensive. The numerical dissipation can be reduced through higher order numerical schemes and finer meshes, however computational costs are further penalized by this. On the other hand, the vortex particle methods are cast into a grid-free Lagrangian formulation and are conservative with respect to vorticity. Thus, they can capture the vortex wake with superior accuracy. However, they have difficulty in treating surface boundary conditions precisely and lack adequate compressibility models.

This doctoral research presents the development of a novel 2-D hybrid inviscid CFD method, which overcomes these difficulties. It is clear that the advantages and disadvantages of Euler/Navier-Stokes methods and vortex particle methods are complementary in nature. Thus, the motivation is to develop a hybrid method which features a fully grided Euler solver in a narrow "gloved" near-field region surrounding the rotor blade, coupled with a grid-free compressible vortex particle method in the 
far-field. It is expected that this type of coupling provides the best of both worlds: Exact treatment of surface boundary conditions and high resolution of flow details near the blade, combined with grid-free and numerical dissipation-free Lagrangian vorticity convection in the far-field. This research is focused in two original directions:

1. the development of a novel compressible formulation for the vortex particle method, which unlike other compressible models available, is applicable to general compressible and vortical rotorcraft flows;

2. the coupling of this compressible formulation with a grid-based Euler solver using the domain decomposition strategy, creating a hybrid method for rotorcraft CFD.

The hybrid blending of a compressible vortex particle method and an Euler solver would be the first of its kind.

\subsubsection{Thesis Outline}

The thesis is organized as follows: Chapter 2 details the formulation of the compressible vortex particle method (CVPM), including the governing equations, particle approximation, the particle-mesh technique and method algorithm. Chapter 3 presents the formulation of the Euler solver, including the governing equations and time and space discretizations. Chapter 4 presents the coupling strategy for the hybrid method (HEPM), including the model, algorithm and boundary condition 
considerations. Chapter 5 provides code validation results, where the convergence of the method is considered, as well as validation with experimental results. The results conclude with some airfoil-vortex interaction (AVI) studies. The thesis is completed by conclusions and recommendations for future work. The appendices include Appendix A for the ENO scheme formulation, Appendix B for Code User Instructions and Appendix C for the Code Flow Diagrams. 


\section{Chapter 2}

\section{The Compressible Vortex Particle Method Formulation}

This chapter provides detailed formulation of the compressible vortex particle method (CVPM). Firstly, the governing flow equations are presented, followed by the detailed description of the candidate methods for the compressible formulation. The selected method is then expanded in detail, completed by a step-by-step solution algorithm.

\subsection{Euler Equations and the Helmholtz Decompo- sition}

Inviscid compressible particle method formulations originate from the Euler equations, which describe the time evolution of inviscid, adiabatic and compressible flow 
as:

$$
\begin{aligned}
\frac{\partial \rho}{\partial t}+\nabla \cdot(\rho \vec{u}) & =0 \\
\frac{\partial \vec{u}}{\partial t}+(\vec{u} \cdot \nabla) \vec{u}+\frac{\nabla p}{\rho} & =0 \\
p & =C \rho^{\gamma}
\end{aligned}
$$

where $\rho, \vec{u}, p, C$ and $\gamma$ are the density, velocity, pressure, a flow constant and the ratio of specific heats ( $\gamma=1.4$ for air at sea level conditions) respectively. For vortex particle methods, these equations are rewritten by using a vector calculus identity and Helmholtz' theorem. The result is the transformation of the Euler equations from an Eulerian frame suitable for grid-based numerical solutions into a form which can take advantage of the Lagrangian frame of reference suitable for particle methods. The new system of equations also explicitly includes the vorticity transport equation.

The transformation begins with the decomposition of the velocity field with the application of Helmholtz' theorem. This states that any sufficiently smooth vector field of compact support may be decomposed into its orthogonal components. In this way, the velocity field is separated into irrotational (curl-free) and vortical (divergence-free) components, i.e.

$$
\vec{u}=\nabla \phi+\nabla \times \vec{\psi}
$$

where $\phi$ and $\vec{\psi}$ are respectively the scalar and vector potentials of the velocity field. Traditional vortex methods assume an incompressible flowfield, including only the 
vortical $(\nabla \times \vec{\psi})$ component of this decomposition. In a compressible formulation, the compressibility effects must be included by considering the scalar potential term $(\nabla \phi)$ as well, representing the irrotational component associated with flow dilatation.

From this point onwards, only two dimensions, $(x, y)$, will be considered. This allows the vorticity vector, $\vec{\omega}$, to be expressed as a scalar where $\vec{\omega}=\omega \vec{k}$, and it is understood that the vorticity field is the curl of the velocity field, $\omega=\nabla \times \vec{u}$, where $\vec{u}=(u, v)^{T}$.

\subsection{Review of Compressible Formulations}

As identified in the literature survey, there are three possible compressible formulations for vortex particle methods: the vorticity, potential and pressure, $(\omega, \phi, p)$, formulation by Meske [37], the dilating vortex particle method (DVPM) of Eldredge [39] and the density, vorticity and potential, $(\rho, \omega, \phi)$, formulation by Mas-Gallic [38]. These will be reviewed in detail in the proceeding sections.

\subsubsection{Meske's $(\omega, \phi, p)$ Formulation}

In Meske's approach [37], the compressible $(\nabla \phi)$ and vortical $(\nabla \times \psi)$ components of the velocity field are related to one another through a pseudo-pressure term, $q$. This method features both particles which track vorticity and an overlying mesh on which compressibility is solved. The formulation is obtained by inserting the Helmholtz 
decomposition (2.4) into the Euler momentum equation (2.2) yielding,

$$
\begin{aligned}
\frac{\partial \phi}{\partial t}+\frac{u^{2}}{2}+\int_{V} \frac{d p}{\rho} & =q \\
\nabla^{2} q & =\nabla \cdot(\vec{u} \times \omega)
\end{aligned}
$$

The continuity equation (2.1) can then be written as:

$$
\frac{1}{\rho} \frac{\partial \rho}{\partial t}+\frac{\vec{u}}{\rho} \cdot \nabla \rho+\nabla \cdot \vec{u}=0
$$

In the above equation, there are three terms which can be transformed separately. For the first term, using equation (2.5) and the relation:

$$
\frac{\partial p}{\partial t}=\frac{\partial p}{\partial \rho} \frac{\partial \rho}{\partial t}=c^{2} \frac{\partial \rho}{\partial t}
$$

we obtain:

$$
\frac{1}{\rho} \frac{\partial \rho}{\partial t}=-\frac{1}{c^{2}}\left[\frac{\partial^{2} \phi}{\partial t^{2}}+\frac{\partial}{\partial t} \frac{u^{2}}{2}-\frac{\partial q}{\partial t}\right]
$$

where $c$ is the speed of sound. For the second term we use:

$$
\frac{\partial \vec{u}}{\partial t}-\vec{u} \times \omega+\nabla\left(\frac{u^{2}}{2}\right)+\frac{1}{\rho} \nabla p=0
$$

and:

$$
\frac{1}{\rho} \nabla p=\frac{c^{2}}{\rho} \nabla \rho=-\frac{\partial \vec{u}}{\partial t}-\nabla\left(\frac{u^{2}}{2}\right)+\vec{u} \times \omega
$$

Multiplying by $\vec{u}$ and knowing that $(\vec{u} \times \omega) \cdot \vec{u}=0$ :

$$
\frac{\vec{u}}{\rho} \cdot \nabla \rho=-\frac{1}{c^{2}}\left[\frac{\partial}{\partial t}\left(\frac{u^{2}}{2}\right)+\vec{u} \cdot \nabla\left(\frac{u^{2}}{2}\right)\right]
$$


The final term is replaced by $\nabla^{2} \phi$ and thus equation (2.6) is transformed into the following wave equation:

$$
\nabla^{2} \phi-\frac{1}{c^{2}}\left[\frac{\partial^{2} \phi}{\partial t^{2}}+\frac{\partial u^{2}}{\partial t}+\vec{u} \cdot \nabla\left(\frac{u^{2}}{2}\right)-\frac{\partial q}{\partial t}\right]=0
$$

The derivatives of the velocity field are decomposed into second derivatives for $\phi$ and $\psi$, with the unknown $\phi$ terms placed on the left hand side:

$$
\begin{array}{r}
\left(c^{2}-u^{2}\right) \frac{\partial^{2} \phi}{\partial x^{2}}-2 u v \frac{\partial^{2} \phi}{\partial x \partial y}+\left(c^{2}-v^{2}\right) \frac{\partial^{2} \phi}{\partial y^{2}}-2 u \frac{\partial^{2} \phi}{\partial x \partial t}-2 v \frac{\partial^{2} \phi}{\partial y \partial t}-\frac{\partial^{2} \phi}{\partial t^{2}} \\
=-u v \frac{\partial^{2} \psi}{\partial x^{2}}+\left(u^{2}-v^{2}\right) \frac{\partial^{2} \psi}{\partial x \partial y}+u v \frac{\partial^{2} \psi}{\partial y^{2}}-2 v \frac{\partial^{2} \psi}{\partial x \partial t}+2 u \frac{\partial^{2} \psi}{\partial y \partial t}-\frac{\partial q}{\partial t}
\end{array}
$$

The remaining unknown is the speed of sound, $c$, which for adiabatic conditions can be written as:

$$
c^{2}=\gamma \frac{p_{\infty}^{\frac{1}{\gamma}}}{\rho_{\infty}} p^{\frac{\gamma-1}{\gamma}}
$$

where $p_{\infty}$ and $\rho_{\infty}$ are the reference pressure and density respectively. For isentropic conditions density is written as a function of pressure as:

$$
\rho=\rho_{\infty}\left(\frac{p}{p_{\infty}}\right)^{\frac{1}{\gamma}}
$$

This allows us to write the pressure derivatives as follows:

$$
\begin{aligned}
-\frac{1}{\rho} \frac{\partial p}{\partial x} & =-\frac{c_{\infty}^{2}}{\gamma-1} \frac{\partial}{\partial x}\left(\left(\frac{p}{p_{\infty}}\right)^{\frac{\gamma-1}{\gamma}}\right) \\
-\frac{1}{\rho} \frac{\partial p}{\partial y} & =-\frac{c_{\infty}^{2}}{\gamma-1} \frac{\partial}{\partial y}\left(\left(\frac{p}{p_{\infty}}\right)^{\frac{\gamma-1}{\gamma}}\right)
\end{aligned}
$$


The pressure term in the Euler momentum equation is replaced and we obtain:

$$
\begin{aligned}
& \frac{\partial u}{\partial t}+u \frac{\partial u}{\partial x}+v \frac{\partial u}{\partial y}=-\frac{c_{\infty}^{2}}{\gamma-1} \frac{\partial}{\partial x}\left(\left(\frac{p}{p_{\infty}}\right)^{\frac{\gamma-1}{\gamma}}\right) \\
& \frac{\partial v}{\partial t}+u \frac{\partial v}{\partial x}+v \frac{\partial v}{\partial y}=-\frac{c_{\infty}^{2}}{\gamma-1} \frac{\partial}{\partial y}\left(\left(\frac{p}{p_{\infty}}\right)^{\frac{\gamma-1}{\gamma}}\right)
\end{aligned}
$$

Taking the derivative of the first equation with respect to $x$, and the derivative with respect to $y$ of the second equation and adding them, while also multiplying by $(\gamma-1) / c_{\infty}^{2}$ we obtain a pressure equation:

$$
\begin{aligned}
-\nabla^{2}\left(\left(\frac{p}{p_{\infty}}\right)^{\frac{\gamma-1}{\gamma}}\right) \frac{c_{\infty}^{2}}{\gamma-1}= & \frac{\partial^{2} u}{\partial x \partial t}+\frac{\partial^{2} v}{\partial y \partial t} \\
& +u \frac{\partial^{2} u}{\partial x^{2}}+v \frac{\partial^{2} u}{\partial x \partial y}+u \frac{\partial^{2} v}{\partial x \partial y}+v \frac{\partial^{2} v}{\partial y^{2}} \\
& +\left(\frac{\partial u}{\partial x}\right)^{2}+2 \frac{\partial v}{\partial x} \frac{\partial u}{\partial y}+\left(\frac{\partial v}{\partial y}\right)^{2}
\end{aligned}
$$

The steps to this method are summarized as follows:

1. Solve Poisson's equation for the vector potential on the particle-mesh to find the rotational component of velocity;

2. Calculate pseudo-pressure on the particle-mesh with equation (2.5);

3. Calculate pressure on the particle-mesh with equation (2.19);

4. Resolve equation (2.13) to obtain potential on the particle-mesh;

5. Resolve vorticity transport equation for the particle method. 
Meske found that this formulation was only successful for cases of constant pseudopressure situations $(d q / d t=0)$. For nonconstant $q$, it was found that there was an amplification of oscillation errors when passing information between particles and mesh.

\subsubsection{Eldredge's Dilating Vortex Particle Method (DVPM)}

The first fully Lagrangian formulation for the full compressible 2-D Navier-Stokes equations was developed by Eldredge [39]. In this method, a standard vortex particle approach is used. However, the particles also carry dilatation, enthalpy, entropy and density; thereby satisfying all the equations of motion. As before, particles are convected according to the local fluid velocity, while particle quantities evolve according to the following transport equations cast in a Lagrangian sense. These equations for vorticity $(\omega)$, dilatation $(\theta)$ and entropy $(s)$ are given as follows:

$$
\begin{gathered}
\frac{D \omega}{D t}=-\omega \theta+\nabla \times(h \nabla s) \cdot \hat{e}_{3}+\frac{1}{R e} \nabla \times\left[\frac{1}{\rho}\left(\frac{4}{3} \nabla \theta-\nabla \times \omega\right)\right] \cdot \hat{e}_{3} \\
\frac{D \theta}{D t}=-\nabla^{2} h-\nabla \vec{u}:(\nabla \vec{u})^{T}+\nabla \cdot(h \nabla s)+\frac{1}{R e} \nabla \cdot\left[\frac{1}{\rho}\left(\frac{4}{3} \nabla \theta-\nabla \times \omega\right)\right] \cdot \hat{e}_{3} \\
\frac{D s}{D t}=\frac{1}{R e} \frac{\Phi}{\rho h}+\frac{1}{R e P r} \frac{1}{\rho h} \nabla^{2} h
\end{gathered}
$$


where $R e$ and $P r$ are the Reynolds' and Prandtl numbers, respectively. The viscous dissipation term, $\Phi$, is expressed as

$$
\Phi=\omega^{2}+2 \nabla \vec{u}:(\nabla \vec{u})^{T}-\frac{2}{3} \theta^{2}
$$

where the : operator denotes a double contraction. Enthalpy, density and entropy are related according to the equation of state

$$
h=\frac{\rho^{\gamma-1}}{\gamma-1} e^{\gamma s}
$$

where $e$ is the internal energy. Finally, particle density is adjusted according to the continuity equation

$$
\frac{D \rho}{D t}=-\rho \theta
$$

In this formulation, Eldredge implements a particle-strength exchange (PSE) technique to treat viscous effects, and the fast multipole method (FMM) to increase computational efficiency. Eldredge's main interest is in aero-acoustics, and hence his tests concern baroclinic vorticity generation, co-rotating vortex pairs and leap-frogging vortices. It is suggested by Eldredge that this method can be easily extended to three dimensions through the inclusion of a vortex stretching term. This method is not considered as a candidate for this work because of its viscous nature, and the difficulty of applying the entropy evolution equation to the rotorcraft situation which will include solid boundaries. 


\subsubsection{Mas-Gallic's $(\rho, \omega, \phi)$ Formulation}

The third method of introducing compressibility in vortex particle methods is that of Mas-Gallic et al [38] referred to as the $(\rho, \omega, \phi)$ formulation. This formulation was originally discussed in the early 1990s as an attractive option for introducing compressibility effects into the general context of vortex methods, and applied to cases of aerodynamic interest in view of implementing a code for flows around solid boundaries. However, it was never coded and validated for vortical flows. Simulations were run for dilating and non-rotating flows with a shock propagation case and results were promising.

Previously, it was found that Meske's $(\omega, \phi, p)$ formulation was only convergent for simulations with constant $q$. Additionally, the pure particle-based DVPM approach of Eldredge is a viscous approach, which includes difficulties associated with the implementation of the entropy condition to a general case, making it prohibitive for practical applications. As such, Mas-Gallic's $(\rho, \omega, \phi)$ approach shows the most promise, and is selected to be expanded and developed for the compressible formulation in the present work. The details of this method are below.

\subsection{Current method: $(\rho, \omega, \phi)$ Formulation}

As with the Meske formulation, the current formulation begins with the Helmholtz decomposition of the velocity field. Substituting equation (2.4) and the vector identity 
$(\vec{u} \cdot \nabla) \vec{u}=\nabla \frac{1}{2}|\vec{u}|^{2}-\vec{u} \times \vec{\omega}$ into equation (2.2), the Euler momentum equation may be written in terms of vorticity and the scalar and vector potentials as

$$
\frac{\partial \vec{w}}{\partial t}-(\nabla \phi+\vec{w}) \times \vec{w}+\nabla\left(\frac{\partial \phi}{\partial t}+\frac{1}{2}|(\nabla \phi+\vec{w})|^{2}+i\right)=0
$$

where $\vec{w}=\nabla \times \vec{\psi}$ is the vortical velocity component, and $i$ is the specific enthalpy. Assuming a two dimensional flowfield, the curl of equation (2.26) yields the vorticity transport equation:

$$
\frac{\partial \vec{\omega}}{\partial t}+\nabla \cdot(\vec{\omega} \vec{u})=0
$$

If we allow the third bracketed term in equation (2.26) to equal $q$, then the divergence of equation (2.26) yields:

$$
\nabla^{2} q-\nabla \cdot(\vec{u} \times \vec{\omega})=0
$$

where the quantity $q$ is given by:

$$
q=\frac{\partial \phi}{\partial t}+\frac{1}{2}|\vec{u}|^{2}+i
$$

Here, $q$, is referred to as the pseudo-pressure term since it is essentially a summation of the thermodynamic pressure (term containing $i$ ) and dynamic pressure (terms containing $\phi$ and $\vec{u}^{2}$ ). The specific enthalpy, $i$, is written in terms of pressure and density with the use of Gibb's equation

$$
T d s=d e+p d\left(\frac{1}{\rho}\right)
$$


where $T$ is temperature, $s$ is entropy and $e$ is the internal energy. Assuming air as a perfect gas and the flow being isentropic, the equation of state and the isentropic relation for pressure and density can be written as:

$$
\begin{aligned}
p & =(\gamma-1) \rho e \\
\frac{p_{\infty}}{\rho_{\infty}^{\gamma}} & =\frac{p}{\rho^{\gamma}}
\end{aligned}
$$

Insert equations (2.31-2.32) into equation (2.30) and integrating yields an expression for the specific enthalpy:

$$
i=C \frac{\gamma}{\gamma-1} \rho^{\gamma-1}
$$

where $C=\frac{p_{\infty}}{\rho_{\infty}^{\gamma}}$ is a flow constant.

Equations (2.1-2.3) can now be transformed into the following system of equations:

$$
\begin{aligned}
\frac{\partial \rho}{\partial t}+\nabla \cdot(\rho \vec{u}) & =0 \\
\frac{\partial \vec{\omega}}{\partial t}+\nabla \cdot(\vec{\omega} \vec{u}) & =0 \\
\nabla^{2} q & =\nabla \cdot(\vec{u} \times \vec{\omega}) \\
q & =\frac{\partial \phi}{\partial t}+\frac{1}{2}|\vec{u}|^{2}+C \frac{\gamma}{\gamma-1} \rho^{\gamma-1}
\end{aligned}
$$

In the above system of equations it is clear that equations (2.34) and (2.35) are equivalent to the statement that mass and circulation are conserved along material elements. These two equations can be written in Lagrangian form as:

$$
\begin{aligned}
& \frac{D M}{D t}=0 \\
& \frac{D \Gamma}{D t}=0
\end{aligned}
$$


where $M=\rho V$ is mass and $\Gamma=\omega V$ is circulation and $V$ is volume. While timeevolution equations must be written in a Lagrangian sense for particle methods, it is evident that the equation for pseudo-pressure, $q$, equation 2.37 should not. This timeevolution equation for the scalar potential, $\phi$, has the form of a Hamilton-Jacobi type wave equation in which information is passed according to the local characteristic speed of the fluid. This is unsuitable for a Lagrangian description which evolves fluid elements according to the local fluid velocity. In principal, this leaves us with a situation where vorticity and density may be tracked along fluid particles, while the flow compressibility effect, or dilatation, is solved for in an Eulerian sense. This requires the use of both particles and a completely overlapping grid, with information being passed between the two in a procedure referred to as the particle-mesh (PM) technique.

\subsection{Particle Approximation}

With the system of governing flow equations developed, it is necessary to present the mathematics involved in the discretization of the flowfield into a series of particles. An in-depth review of the particle approximation of a flowfield may be found in Cottet and Koumoutsakos [27]. The following is a brief overview of the formulation.

The basic idea in traditional vortex particle methods is to first initialize the flow on a regular lattice on which vorticity is considered concentrated on single markers or particles. The vorticity is then recovered from the particles through the following 
convolution

$$
\omega(\vec{x}, t)=\sum_{p} \omega_{p} V_{p} \delta_{c}\left[\vec{x}-\vec{x}_{p}(t)\right]
$$

where $p$ particles, each located at $\vec{x}_{p}$ at time $\mathrm{t}$, are carrying vorticity, $\omega_{p}$, and have associated volumes, $V_{p}$. Here, $\delta_{c}$ denotes the Dirac measure. In the compressible vortex method, particles will also have an associated dilatation, $\delta_{p}$, and density, $\rho_{p}$, whose fields are similarly recovered by

$$
\begin{aligned}
& \delta(\vec{x}, t)=\sum_{p} \delta_{p} V_{p} \delta_{c}\left[\vec{x}-\vec{x}_{p}(t)\right] \\
& \rho(\vec{x}, t)=\sum_{p} \rho_{p} V_{p} \delta_{c}\left[\vec{x}-\vec{x}_{p}(t)\right]
\end{aligned}
$$

The velocity field reconstruction is done by first considering the Poisson's equations for the scalar and vector potentials

$$
\begin{aligned}
\nabla^{2} \phi & =\delta \\
\nabla^{2} \psi & =-\omega
\end{aligned}
$$

The above equations can be solved with the aid of a Green's function constructed for the applicable linear differential operator. In the presence of both volume and surface dilatation and vorticity, this solution is written as the vector Poincare identity 
of the potential fields:

$$
\begin{aligned}
\phi(\vec{x}) & =-\int_{\vec{x}^{\prime}} \delta\left(\vec{x}^{\prime}\right) G(\vec{r}) d \vec{x}^{\prime}-\int_{\vec{x}_{s}^{\prime}} u_{n}\left(\vec{x}_{s}^{\prime}\right) G\left(\vec{r}_{s}\right) d \vec{x}_{s}^{\prime} \\
\psi(\vec{x}) & =\int_{\vec{x}^{\prime}} \omega\left(\vec{x}^{\prime}\right) G(\vec{r}) d \vec{x}^{\prime}+\int_{\vec{x}_{s}^{\prime}} u_{t}\left(\vec{x}_{s}^{\prime}\right) G\left(\vec{r}_{s}\right) d \vec{x}_{s}^{\prime}
\end{aligned}
$$

Here $G(\vec{x})=-\frac{1}{2 \pi} \ln |\vec{x}|$ is the Green's function for the inverse Laplacian and is evaluated at $\vec{r}=\vec{x}-\vec{x}^{\prime}$ and $\vec{r}_{s}=\vec{x}-\vec{x}_{s}^{\prime}$. Here $\vec{x}^{\prime}$ denotes the flow volume locations, $\vec{x}_{s}$ denotes surface locations and $u_{n}$ and $u_{t}$ are the normal and tangential surface disturbance velocities respectively. Substituting the above solutions for the potentials into the Helmholtz decomposition (2.4) yields an integral equation for the velocity field.

$$
\begin{aligned}
\vec{u}(\vec{x})=-\int_{\vec{x}^{\prime}} \delta\left(\vec{x}^{\prime}\right) \vec{K}(\vec{r}) d \vec{x}^{\prime}-\int_{\vec{x}_{s}^{\prime}} u_{n}\left(\vec{x}_{s}^{\prime}\right) \vec{K}\left(\vec{r}_{s}\right) d \vec{x}_{s}^{\prime} & \\
& +\int_{\vec{x}^{\prime}} \omega\left(\vec{x}^{\prime}\right) \vec{k} \times \vec{K}(\vec{r}) d \vec{x}^{\prime}+\int_{\vec{x}_{s}^{\prime}} u_{t}\left(\vec{x}_{s}^{\prime}\right) \vec{k} \times \vec{K}\left(\vec{r}_{s}\right) d \vec{x}_{s}^{\prime}
\end{aligned}
$$

which can be interpreted as the Biot-Savart law in addition to its counterpart in dilatation. Here, $\vec{K}$, is the velocity kernel defined as

$$
\vec{K}(\vec{x})=\nabla G(\vec{x})=-\frac{\vec{x}}{2 \pi|\vec{x}|^{2}}
$$

It is clear that the velocity kernel becomes highly singular as particles approach one another. To overcome this difficulty, it is necessary to add some "mollification", which essentially means the addition of a finite core to the particles, transforming the singular particle to a smoothed "blob" entity. Figure 2.1 demonstrates the singularity 
in this unmollified kernel, along with two mollifications of increasing accuracy where the smoothing is associated with a blob core size, $\varepsilon$.

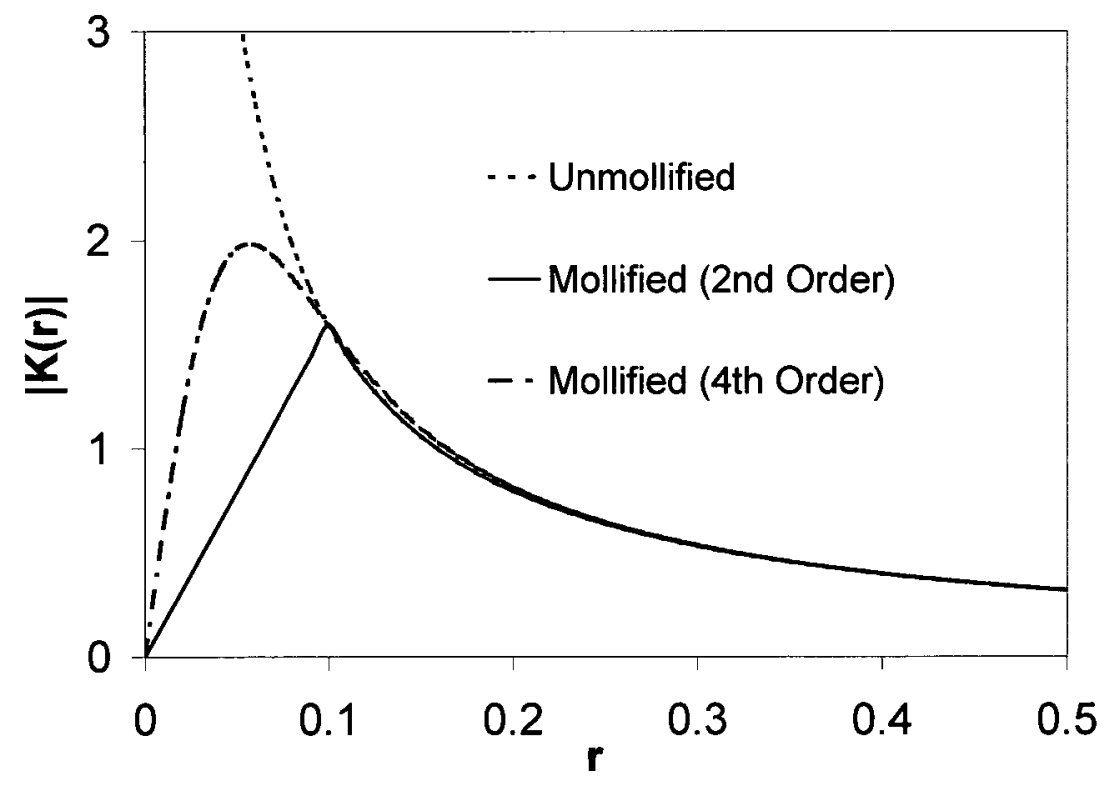

Figure 2.1: Velocity kernel distribution for mollified and unmollified kernels $(\varepsilon=0.1)$.

Mathematically, this smoothing is accomplished by the convolution of the velocity kernel in equation (2.48) with a blob cut-off function.

$$
\vec{K}_{\varepsilon}=\vec{K} \star \zeta_{\varepsilon}
$$

The convergence of vortex blob methods was proved independently by Hald [42], Beale and Majda [43], Raviart [44] and Anderson and Greengard [45]. In each it is proven that errors in the method are due to the interpolation of the velocity field by particles and by the choice in accuracy for $\zeta_{\varepsilon}$. Although no additional error should be present due to the inclusion of the dilatation component of the vector field, it is 
necessary for method convergence to ensure some measure of particle overlap. This requires that the ratio of particle radius to particle spacing, $\kappa=\varepsilon / \Delta h$, must be greater than one. The smoothing error is determined by the choice of accuracy for the blob cut-off function, which is constructed by satisfying the following moment conditions:

$$
\begin{aligned}
\int \zeta(\vec{x}) d \vec{x} & =1 \\
\int \vec{x}^{i} \zeta(\vec{x}) d \vec{x} & =0 \text { if }|i| \leq r-1 \\
\int|\vec{x}|^{r}|\zeta(\vec{x})| d \vec{x} & <\infty
\end{aligned}
$$

The above conditions signify that the cut-off has, up to the power of $(r-1)$, the same moment properties as the Dirac measure. This means that the blobs share the same momentum, starting with circulation, linear impulse, and so on, up to the order of $(r-1)$ as the particles in (2.40-2.41). Table 2.1 displays several examples of blob functions and associated mollified velocity kernels of increasing orders of accuracy.

Table 2.1: Blob functions and mollified velocity kernels $[r=(x, y)]$

\begin{tabular}{ccc}
\hline \hline Order & $\zeta$ & $K_{\varepsilon}$ \\
\hline & $\frac{1}{2 \pi r} r \leq 1$ & $\frac{(x, y)}{2 \pi \varepsilon} r \leq \varepsilon$ \\
2 & $0 \quad r>1$ & $\frac{(x, y)}{2 \pi r^{2}} r>1$ \\
4 & $\frac{2}{\pi} \frac{2-r^{2}}{\left(1+r^{2}\right)^{4}}$ & $\frac{(x, y)}{2 \pi} \frac{\left.r^{4}+3(\varepsilon r)^{2}+4 \varepsilon^{4}\right)}{\left(\varepsilon^{2}+r^{2}\right)^{3}}$ \\
6 & $\frac{1}{\pi}\left(6-6 r^{2}+r^{4}\right) e^{-r^{2}}$ & $\frac{(x, y)}{2 \pi r^{2}}\left[1-\left(1-2 \frac{r^{2}}{\varepsilon^{2}}+\frac{r^{4}}{2 \varepsilon^{4}}\right)\right] e^{\frac{-r^{2}}{\varepsilon^{2}}}$
\end{tabular}


The cut-off function is then finalized with the aid of the cut-off length, $\varepsilon$, according

to

$$
\zeta_{\varepsilon}(\vec{x})=\varepsilon^{-2} \zeta\left(\frac{\vec{x}}{\varepsilon}\right)
$$

Finally, the field reconstruction equations are written in discrete form as:

$$
\begin{aligned}
\rho(\vec{x}, t) & =\sum_{p} M_{p} \zeta_{\varepsilon}\left(\vec{x}-\overrightarrow{x_{p}}(t)\right) \\
\delta(\vec{x}, t) & =\sum_{p} \Delta_{p} \zeta_{\varepsilon}\left(\vec{x}-\overrightarrow{x_{p}}(t)\right) \\
\omega(\vec{x}, t) & =\sum_{p} \Gamma_{p} \zeta_{\varepsilon}\left(\vec{x}-\overrightarrow{x_{p}}(t)\right)
\end{aligned}
$$

where $\Delta_{p}=\delta_{p} V_{p}$ is the particle divergence. The velocity field is recovered by:

$$
\begin{aligned}
\vec{u}(\vec{x})=-\sum_{p} \Delta_{p} \vec{K}_{\varepsilon}(\vec{r}) & -\sum_{s} u_{n_{s}} L_{s} \vec{K}_{\varepsilon}\left(\vec{r}_{s}\right) \\
& +\sum_{p} \Gamma_{p} \vec{k} \times \vec{K}_{\varepsilon}(\vec{r})+\sum_{s} u_{t_{s}} L_{s} \vec{k} \times \vec{K}_{\varepsilon}\left(\vec{r}_{s}\right)
\end{aligned}
$$

where $L_{s}$ is the length of the airfoil surface segment associated with the given surface disturbance velocity.

The particle field is deformed according to an Adams-Bashforth time integration scheme shown in equations (2.59-2.60). This is a 4 th order scheme dependent on the gradients from three previous timesteps. As such, upon method initialization, it is necessary to invoke three forward Euler timesteps in order to develop the necessary gradient history to implement the Adams-Bashforth scheme.

From the governing equations (2.34-2.37) it is seen that particle mass and circulation are conserved. In order to preserve continuity, the particle volumes must account 
for dilatation

$$
\frac{D V_{p}}{D t}=\Delta_{p}
$$

As such, particles are convected according to the local flow velocity and their volumes are modified according to the local flow dilatation according to:

$$
\begin{gathered}
\vec{X}_{p}^{n+1}=X_{p}^{n}+\frac{1}{24}\left[55 \vec{u}\left(\vec{X}_{p}^{n}\right)-59 \vec{u}\left(\vec{X}_{p}^{n-1}\right)+37 \vec{u}\left(\vec{X}_{p}^{n-2}\right)-9 \vec{u}\left(\vec{X}_{p}^{n-3}\right)\right] d t \\
V_{p}^{n+1}=V_{p}^{n}+\frac{1}{24}\left[55 \Delta\left(\vec{X}_{p}^{n}\right)-59 \Delta\left(\vec{X}_{p}^{n-1}\right)+37 \Delta\left(\vec{X}_{p}^{n-2}\right)-9 \Delta\left(\vec{X}_{p}^{n-3}\right)\right] d t
\end{gathered}
$$

Although particle mass and circulation are constant, the changes in particle volume necessitate a subsequent change in particle density and vorticity. Along with the $q$ term, this is how density and vorticity are dynamically coupled with flow compressibility.

\subsection{Particle-Mesh Technique}

Implementation of a particle method with the direct integral formulation of the preceding section is extremely prohibitive. The velocity calculation contains a convolution over the entire particle field, which requires $O\left(N^{2}\right)$ computations, where $N$ is the number of particles. In practice, computer limitations would not allow more than only a few thousand particles with this method, which is insufficient to model any real-world situation. To avoid this computational penalty, the approach taken is to implement a particle-mesh (PM) technique summarized by Huberson and Voutsinas [30]. In a PM approach, particle values are projected onto an orthogonal grid, 
on which the velocity calculation can be done very efficiently using a Poisson solver based on a Fast Fourier Transform (FFT) [46]. Once the velocity is calculated, it may be interpolated back to the particles, which are subsequently convected. This approach significantly reduces the computational cost to $O(N \log N)$, which makes large engineering problems tractable. In this CVPM formulation the PM serves two purposes. Not only does it allow for efficient computations, but it is also used as the overlying Eulerian grid, on which the compressibility effects may be calculated.

\subsubsection{Projection/Interpolation Operators}

In order to implement this technique, it is necessary to define an operator which allows the transfer of information between the particles and the grid. For the case of the projection of a general particle value, $f$, onto an orthogonal PM consisting of nodes $\vec{x}_{i j}$ we can write:

$$
f\left(\vec{x}_{i j}\right)=\sum_{p} f_{p} \Phi\left(\vec{X}_{p}-\vec{x}_{i j}\right)
$$

where $\Phi$ is an interpolation function which serves the same purpose as $\zeta_{\varepsilon}$, but is built on a grid according to

$$
\Phi(\vec{X})=w_{x}\left(\frac{X-x_{i j}}{h}\right) w_{y}\left(\frac{Y-y_{i j}}{h}\right)
$$

where $h$ is the PM grid dimension. Refer to Cottet and Koumoutsakos [27] for examples of interpolation functions along with their construction methods. The function 
selected for the present work was the 3rd order Triangular-Shaped Cloud (TSC) function written as:

$$
w_{x}=\left\{\begin{array}{ll}
0, & \text { if }|x|>3 / 2 \\
\frac{1}{2}(-|x|+3 / 2)^{2}, & \text { if } 1 / 2 \leq|x| \leq 3 / 2 \\
\frac{1}{2}(x+3 / 2)^{2}-\frac{3}{2}(x+1 / 2)^{2} & \text { if } 0 \leq|x| \leq 1 / 2
\end{array}\right\}
$$

This function was selected because it is the highest order interpolation possible which retains positivity over its support. The above interpolation procedure does not account for the non-uniformities in the particle space distribution or particle volumes. Gingold and Monaghan [47] solved this issue by also including the volume/sparsity effect in the interpolation:

$$
f\left(\vec{x}_{i j}\right)=\frac{\sum_{p} f_{p} V_{p} \Phi\left(\vec{X}_{p}-\vec{x}_{i j}\right)}{\sum_{p} V_{p} \Phi\left(\vec{X}_{p}-\vec{x}_{i j}\right)}
$$

The transferral of information from particles to the PM is referred to as a projection, where the projection of a particle value, $f_{p}$, onto the PM is written mathematically as $\operatorname{Proj}\left(f_{p}\right)$. The reverse transformation, from $\mathrm{PM}$ to particles is referred to as an interpolation, written as $\operatorname{Interp}\left(f_{i j}\right)$. The equation for this interpolation is given as

$$
f\left(\vec{x}_{p}\right)=\sum_{i j} f_{i j} \Phi\left(\vec{X}_{p}-\vec{x}_{i j}\right)
$$

and the particle is assigned a volume integrated quantity

$$
F_{p}=f_{p} V_{p}
$$




\subsubsection{Hamilton-Jacobi Equation}

The compressibility effects are also calculated directly on the PM with the solution of the Hamilton-Jacobi equation (2.37) which is rewritten here for clarity:

$$
\frac{\partial \phi}{\partial t}+\frac{1}{2}|\vec{u}|^{2}+C \frac{\gamma}{\gamma-1} \rho^{\gamma-1}=q
$$

This equation is fully expanded with the Helmholtz decomposition (2.4) as:

$$
\frac{\partial \phi}{\partial t}+\frac{1}{2} \nabla \phi^{2}+\vec{w} \cdot \nabla \phi=q-C \frac{\gamma}{\gamma-1} \rho^{\gamma-1}-\frac{1}{2} \vec{w}^{2}
$$

As previously stated, this has the form of a Hamilton-Jacobi hyperbolic equation plus an advection term:

$$
\frac{\partial \phi}{\partial t}+H(\nabla \phi)+\vec{w} \cdot \nabla \phi=f
$$

where the Hamiltonian is defined as:

$$
H(\nabla \phi)=\frac{1}{2} \nabla \phi^{2}
$$

There are many solution techniques available for this type of equation. For this work, it is expected that there will exist discontinuities in $\phi$ at shock occurrences and at the surface of the airfoil. As such, the implementation of a standard centered finite difference scheme would result in spurious oscillations in the areas surrounding the discontinuities. Taking this into consideration, the Essentially Non-Oscillatory (ENO) scheme by Osher and Shu [48] is implemented. The formulation is fully presented in 
Appendix A. At each step, the new potential field is differentiated according to the Poisson's equation (2.43) to obtain an updated dilatation field, which is subsequently interpolated onto the particles prior to the next step.

\subsection{CVPM Algorithm}

In the preceding sections, the CVPM formulation was derived, including a particle approximation for the compressible field, a PM technique for efficient computations and the evolution of flow compressibility. In this section, the solution algorithm implementing the preceding approach is presented.

Given particles carrying mass, circulation, divergence and volume, $(M, \Gamma, \Delta, V)_{p}^{n}$, and assuming that they provide adequate coverage of the particle-mesh, the following solution steps shall be followed. Note that in this procedure below, subscript $(\ldots)_{p}$ denotes particle solutions, while subscript $(\ldots)_{p m}$ denotes particle-mesh solutions and $(\ldots)_{i j}$ denotes a quantity at particle-mesh node $i j$. Also, superscript $(\ldots)^{n}$ denotes values at timelevel $n$ and superscript $(\ldots)^{n+1}$ denotes timelevel $(n+1)$.

1. Project particle values onto PM:

$$
\operatorname{Proj}(M, \Gamma, \Delta, V)_{p}^{n} \Rightarrow(\rho, \omega, \delta)_{p m}^{n}
$$

2. Solve Poisson's equation on PM to obtain grid potentials:

$$
\begin{aligned}
& \nabla^{2} \phi_{i j}^{n}=\delta_{i j}^{n} \\
& \nabla^{2} \psi_{i j}^{n}=-\omega_{i j}^{n}
\end{aligned}
$$


3. Obtain PM velocity:

$$
\vec{u}_{i j}^{n}=\nabla \phi_{i j}^{n}+\nabla \times \psi_{i j}^{n}
$$

4. Interpolate calculated velocity onto the particles:

$$
\operatorname{Interp}(\vec{u})_{p m}^{n} \Rightarrow \vec{u}_{p}^{n}
$$

5. Convect particle field:

$$
\begin{aligned}
& \vec{x}_{p}^{n+1}=\vec{x}_{p}^{n}+\vec{u}_{p}^{n} d t \\
& \vec{V}_{p}^{n+1}=\vec{V}_{p}^{n}+\Delta_{p}^{n} d t
\end{aligned}
$$

6. Solve Poisson's equation for pseudo-pressure on PM:

$$
\nabla^{2} q_{i j}^{n}=\vec{u}_{i j}^{n} \times \vec{\omega}_{i j}^{n}
$$

7. Solve Hamilton-Jacobi equation with ENO scheme to obtain new dilatation on

PM:

$$
\begin{aligned}
& \frac{\partial \phi}{\partial t}+H(\nabla \phi)+\vec{w} \cdot \nabla \phi=f \\
& \delta_{i j}^{n+1}=\nabla^{2} \phi_{i j}^{n+1}
\end{aligned}
$$

8. Interpolate new dilatation onto particles:

$$
\begin{aligned}
& \text { Interp }(\delta)_{p m}^{n+1} \Rightarrow \delta_{p}^{n+1} \\
& \Delta_{p}^{n+1}=\delta_{p}^{n+1} V_{p}^{n+1}
\end{aligned}
$$

9. next timestep $\rightarrow$ return to step $1($ shift $(n+1) \rightarrow n)$ 


\section{Chapter 3}

\section{Finite-Volume Euler Solver}

As outlined in the introduction section, the goal of this thesis is to develop a hybrid, compressible and inviscid CFD model. This will inherently require a classical gridbased CFD method for solving the near-blade flowfield, for which the Carleton MultiBlock (CMB) code has been selected. This is a parallel finite-volume (FV) multi-block solver which uses the MPI parallel protocol. Due to the two-dimensional nature of the coupling, only one processor was implemented at this stage of the work. Also, since this thesis focuses on inviscid code development, only the Euler solver module of the CMB was considered. The following is an overview of CMB's formulation. More detailed derivations and numerical validation may be found in [49-51]. 


\subsection{Euler Equations}

The two dimensional Euler equations in Cartesian coordinates $(x, y)$ can be written in non-dimensional conservative form as

$$
\frac{\partial \vec{W}}{\partial t}+\frac{\partial \vec{F}}{\partial x}+\frac{\partial \vec{G}}{\partial y}=0
$$

where $\vec{W}$ is the vector of conservative variables

$$
\vec{W}=\left\{\begin{array}{c}
\rho \\
\rho U \\
\rho V \\
\rho E
\end{array}\right\}
$$

while $\vec{F}$ and $\vec{G}$ are the flux vectors

$$
\begin{gathered}
\vec{F}=\left\{\begin{array}{c}
\rho U \\
\rho u U+p \\
\rho v U \\
U(\rho E+p)+x_{t} p
\end{array}\right\} \\
\vec{G}=\left\{\begin{array}{c}
\rho V \\
\rho u V \\
\rho v V+p \\
V(\rho E+p)+y_{t} p
\end{array}\right\}
\end{gathered}
$$

where $\rho, u, v, p$ and $E$ are density, Cartesian velocity components, pressure and specific total energy respectively. The terms $U$ and $V$ are the contravariant velocities defined by

$$
\begin{aligned}
U & =u-x_{t} \\
V & =v-y_{t}
\end{aligned}
$$


where $x_{t}$ and $y_{t}$ are the grid speeds in the Cartesian directions. The flow quantities are related to each other by the following perfect gas relations:

$$
\begin{gathered}
E=e+\frac{1}{2}\left(u^{2}+v^{2}\right) \\
p=(\gamma-1) \rho e
\end{gathered}
$$

It should be noted that the quantities presented here have been non-dimensionalized according to the following procedure:

$$
\begin{array}{lll}
x=\frac{x^{*}}{L^{*}} & y=\frac{y^{*}}{L^{*}} & t=\frac{V_{\infty}^{*} t^{*}}{L^{*}} \\
u=\frac{u^{*}}{V_{\infty}^{*}} & v=\frac{v^{*}}{V_{\infty}^{*}} & \rho=\frac{\rho^{*}}{\rho_{\infty}^{*}} \\
p=\frac{p^{*}}{\rho_{\infty}^{*} V_{\infty}^{* 2}} & e=\frac{e^{*}}{V_{\infty}^{* 2}}
\end{array}
$$

where dimensioned quantities are denoted by $*$, while non-dimensional quantities have no superscript.

\subsection{Time and Space Discretization}

The equations are discretized using a cell-centered finite volume method that transforms the partial differential equations into a set of ordinary differential equations which can be written as:

$$
\frac{\partial}{\partial t}\left(V_{i, j} \vec{W}_{i, j}\right)=-\vec{R}_{i, j}(\vec{W})
$$

where $V_{i, j}, \vec{W}_{i, j}$ and $\vec{R}_{i, j}(\vec{W})$ are the volume, vector of conserved variables and the flux residual for the cell $(i, j)$, respectively. The convective fluxes are discretized 
using Osher's upwind flux difference splitting scheme, with a MUSCL [52] variable extrapolation to provide 3rd order accuracy in space. The Van Albada [53] limiter is used to provide monotonic solutions near shock waves. Discretization of equation (3.7) in time by using a fully implicit approach yields

$$
\frac{\vec{W}_{i, j}^{n+1}-\vec{W}_{i, j}^{n}}{\Delta t}=-\frac{1}{V_{i, j}} \vec{R}_{i, j}\left(\vec{W}_{i, j}^{n+1}\right)
$$

Following Jameson [54], the time derivative is discretized with a second order backwards finite difference yielding

$$
\frac{3 V_{i, j}^{n+1} \vec{W}_{i, j}^{n+1}-4 V_{i, j}^{n} \vec{W}_{i, j}^{n}+V_{i, j}^{n-1} \vec{W}_{i, j}^{n-1}}{2 \Delta t}+\vec{R}_{i, j}\left(\vec{W}_{i, j}\right)=0
$$

This equation is nonlinear and cannot be solved analytically. At this point we introduce an unsteady residual defined as

$$
R_{i, j}^{*}\left(\vec{W}_{i, j}^{n+1}\right)=\frac{3 V_{i, j}^{n+1} \vec{W}_{i, j}^{n+1}-4 V_{i, j}^{n} \vec{W}_{i, j}^{n}+V_{i, j}^{n-1} \vec{W}_{i, j}^{n-1}}{2 \Delta t}+\vec{R}_{i, j}\left(\vec{W}_{i, j}\right)=0
$$

This new equation can be seen as the solution to a steady-state problem which can be solved by a time integration through pseudo-time $t^{*}$

$$
\frac{\partial \vec{W}_{i, j}^{n+1}}{\partial t^{*}}+\frac{1}{V_{i, j}} \vec{R}_{i, j}^{*}(\vec{W})=0
$$

whose steady solution satisfies

$$
\frac{\partial \vec{W}_{i, j}^{n+1}}{\partial t^{*}}=0
$$

which means it also must satisfy $\vec{R}_{i, j}^{*}(\vec{W})=0$ and hence must also be the solution to the unsteady problem (3.10). It is therefore possible to take any unsteady real 
time level solution as an integration through pseudo-time to a pseudo-steady-state. This procedure is illustrated in Figure 3.1. This pseudo-time problem can now be solved by any method designed for steady-state problems, including any acceleration techniques.

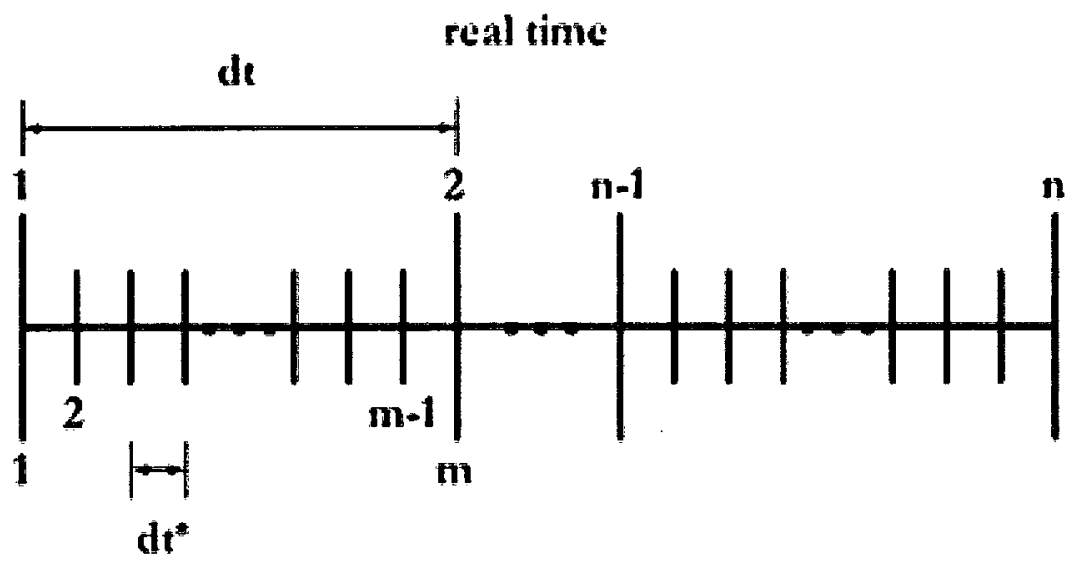

pseudo-time

Figure 3.1: Illustration of implementation of pseudo and real time integration in CMB.

In equation (3.11) the flux residual on the right hand side is written in terms of the unknown solution at this time level. To get an expression in terms of known quantities, i.e. the solution at the previous time level, the flux residual is linearized 
with respect to $t^{*}$.

$$
\begin{aligned}
\vec{R}_{i, j}^{*}\left(\vec{W}^{m+1}\right) & =\vec{R}_{i, j}^{*}\left(\vec{W}^{m}\right)+\frac{\partial \vec{R}_{i, j}^{*}}{\partial t^{*}} \Delta t^{*}+O\left(\Delta t^{* 2}\right) \\
& \approx \vec{R}_{i, j}^{*}\left(\vec{W}^{m}\right)+\frac{\partial \vec{R}_{i, j}^{*}}{\partial \vec{W}_{i, j}} \frac{\partial \vec{W}_{i, j}}{\partial t^{*}} \Delta t^{*} \\
& \approx \vec{R}_{i, j}^{*}\left(\vec{W}^{m}\right)+\frac{\partial \vec{R}_{i, j}^{*}}{\partial \vec{W}_{i, j}} \Delta \vec{W}_{i, j}
\end{aligned}
$$

where $\Delta \vec{W}=\vec{W}^{m+1}-\vec{W}^{m}$, and according to equation (3.10)

$$
\frac{\partial \vec{R}^{*}}{\partial \vec{W}}=\frac{\partial \vec{R}}{\partial \vec{W}}+\frac{3 V}{2 \Delta t} \vec{I}
$$

results in a system of linear equations of the form $A x=b$ as follows:

$$
\left[\left(\frac{V}{\Delta t^{*}}+\frac{3 V}{2 \Delta t}\right) \vec{I}-\frac{\partial \vec{R}}{\partial \vec{W}}\right] \Delta \vec{W}=-\vec{R}^{*}\left(\vec{W}^{m}\right)
$$

The above linear system can be solved by a number of numerical methods. In CMB, the Generalized Conjugant Gradient (GCG) method has been applied. The GCG method provides an approximate solution of a linear system through the minimization of an error function in a finite-dimensional space of potential solution vectors. The preconditioning strategy for the linear system is based on a block incomplete lower-upper (BILU) factorization. This is used because the sparsity pattern of the BILU method is the same as that of the Jacobian matrix. Since implicit schemes require special attention upon initialization, the flowfield is initially solved explicitly before switching to the less robust but much faster implicit method. 


\section{Chapter 4}

\section{Hybrid Euler-Particle Method (HEPM)}

The previous chapters outlined the formulation of the Compressible Vortex Particle Method (CVPM) and of a conventional grid-based Euler solver, respectively. This chapter addresses the coupling strategy employed in mating the two methods into the Hybrid Euler-Particle Method (HEPM). The following sections describe the hybrid model, coupling algorithm and some numerical considerations including non-reflective boundary conditions.

\subsection{HEPM Model}

Figure 4.1 illustrates the key idea of the hybrid method. The model features a conventional finite-volume (FV) grid that has been reduced to a narrow region surrounding the airfoil. This grid will be referred to as the Euler domain, on which the Euler solver (CMB) is implemented. Overlapping this Euler domain is a particle-mesh (PM) domain intended for complete particle coverage. In incompressible vortex methods, 
particles are only required in regions supporting vorticity. However, a compressible formulation also requires particles carrying mass, and thus particles are required throughout the whole domain in order to maintain mass continuity. This type of coupling can be thought of as an overlapping domain decomposition technique, outlined in the literature review, with complete overlap of the two numerical methods. In the construction of the hybrid model, it is important to consider the isentropic nature of the CVPM, requiring the Euler domain to have sufficient extension to encompass all expected shocks. Simulations with the stand-alone Euler solver reveal that the largest expected shocks for rotorcraft applications extend 0.8 chord lengths from the airfoil. In addition, there was found to be convergence issues as the Euler domain reduced to below $3 / 4$ of a chord length in size. As such, it is suggested that a reduced Euler domain of 1 chord extent is sufficient for all expected scenarios in this work.

The outer extent of the PM is limited solely by computational costs, which increase rapidly with the size of the PM. Numerical experiments have shown that the PM should extend at least one chord length from the outer boundaries of the Euler domain.

The left hand side of the model is the inlet to the PM. There is a buffer zone of particles which continuously feeds mass into the PM domain, thereby ensuring continuity. For airfoil-vortex interaction (AVI) studies for example, vorticity can also enter from this direction.

The right hand side of the model is the outlet. Due to Kelvin's circulation theorem, 
which states that for an inviscid, barotropic fluid with conservative body forces the circulation around a closed curve moving with the fluid remains constant with time, it is impossible to simply delete vorticity as it leaves the PM. For this reason, particles which have been identified as carrying vorticity are retained and allowed to convect according to the freestream velocity. To minimize the computational cost of this region, groups of particles are merged together once they are identified to have traveled a sufficient distance from the outlet.

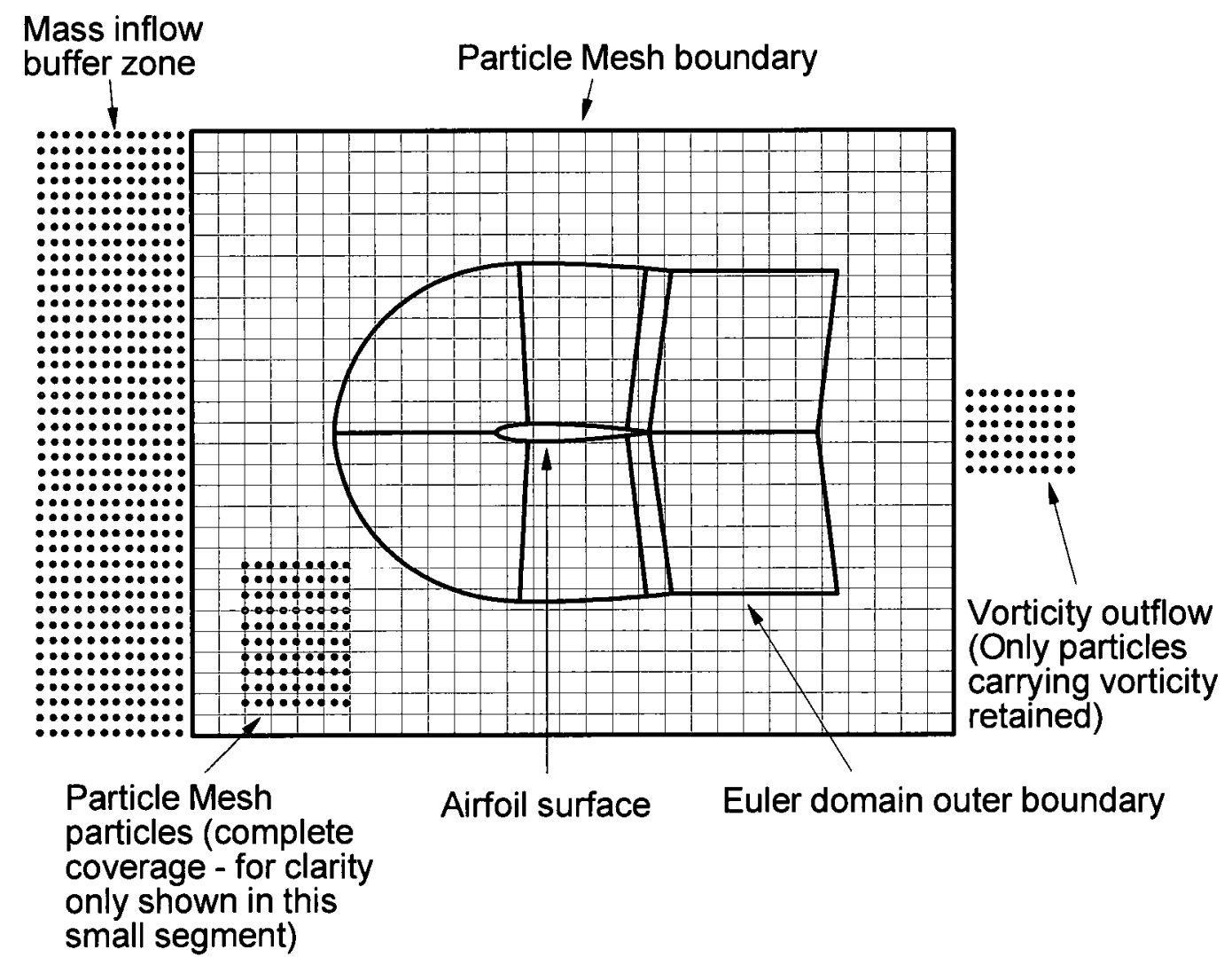

Figure 4.1: HEPM model. 


\subsection{Coupling Algorithm}

At this stage, it is necessary to develop a coupling scheme blending the CVPM outlined in Chapter 2 with the Euler solver whose formulation is outlined in Chapter 3. The key idea of the coupling is to ensure that at the completion of each timestep it can be said that the PM solution is equivalent to the Euler solution. The criteria for this solution compatibility are illustrated in Figure 4.2.

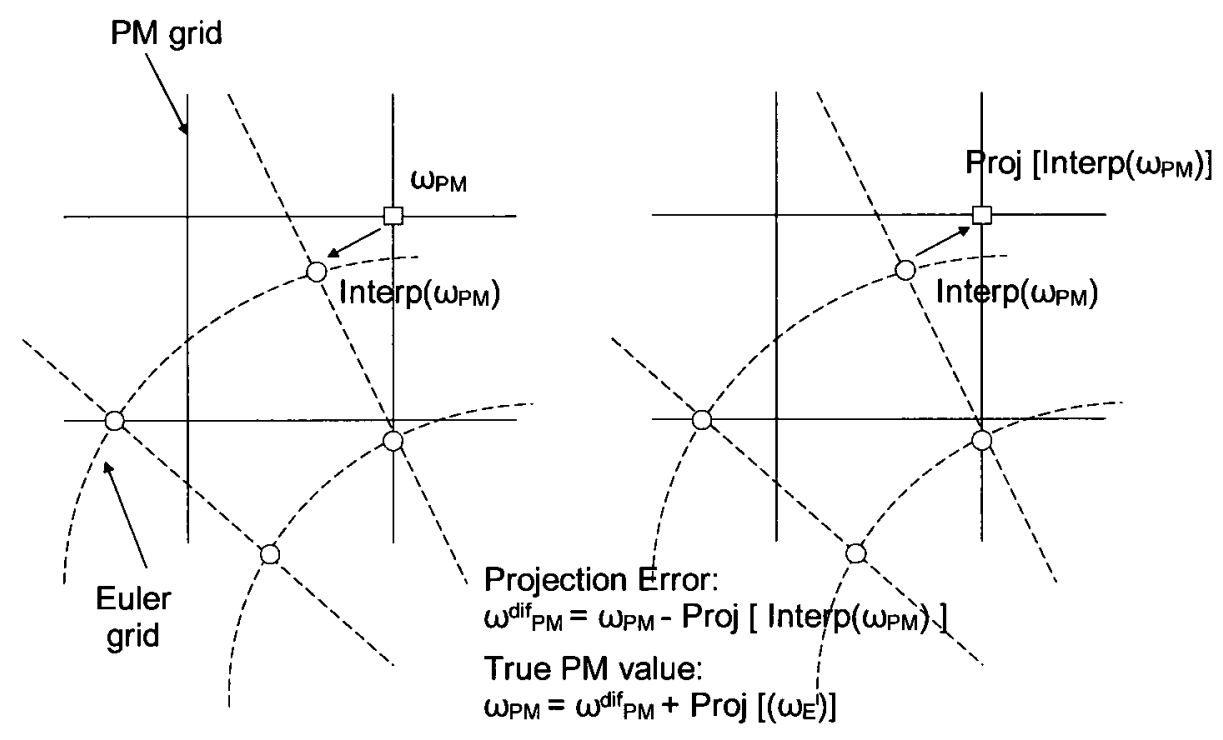

Figure 4.2: Illustration of PM to Euler grid compatibility.

Consider that we have all PM variables defined on the PM grid nodes as obtained at the end of a convection step. Then, one can interpolate those values to the Euler grid boundary locations and solve the Euler equations. The resulting vorticity and dilatation solution in the Euler domain should not be compatible to the current 
PM values due to the fact that the particles have moved and no longer satisfy the airfoil surface boundary conditions. This is not important with respect to the Euler boundary values, because they are located at a far enough distance from the surface to have little impact. However, it is important with regards to the Hamilton-Jacobi equation, which is solved over the whole PM domain. Instead of using buffer zones, the incorrect particle contributions are subtracted. Based on the PM solution, it is possible to interpolate onto the Euler grid and have an Euler equivalent to the PM solution. This gives us $(\hat{\omega}, \hat{\delta}, \hat{\rho})_{E}=\operatorname{Interp}(\omega, \delta, \rho)_{P M}$. Since the PM solution is not correct anyways, we can obtain the PM corrections $(\hat{\omega}, \hat{\delta}, \hat{\rho})_{P M}$ by subtracting the projections of the interpolations $\operatorname{Proj}\left(\operatorname{Interp}(\omega, \delta, \rho)_{P M}\right)$. This gives us:

$$
(\hat{\omega}, \hat{\delta}, \hat{\rho})_{P M}=(\omega, \delta, \rho)_{P M}-\operatorname{Proj}\left(\operatorname{Interp}(\omega, \delta, \rho)_{P M}\right)
$$

and the true PM values as:

$$
(\omega, \delta, \rho)_{P M}=(\hat{\omega}, \hat{\delta}, \hat{\rho})_{P M}+\operatorname{Proj}\left(\operatorname{Interp}(\omega, \delta, \rho)_{P M}\right)
$$

The coupling algorithm is then summarized as follows:

Step 0: Determine the projection error

1. Assume a PM solution $(\omega, \delta, \rho)_{P M}^{0}$

2. Interpolate the PM solution onto the Euler grid:

$$
(\hat{\omega}, \hat{\delta}, \hat{\rho})_{E}=\operatorname{Interp}(\omega, \delta, \rho)_{P M}^{0}
$$


3. Determine projection error by subtracting the projection of the above interpolation:

$$
\left.(\omega, \delta, \rho)_{P M}^{d i f}=(\omega, \delta, \rho)_{P M}^{0}-\operatorname{Proj}(\hat{\omega}, \hat{\delta}, \hat{\rho})_{E}\right)
$$

Step 1: $\mathrm{PM} \leftrightarrow$ Euler Iterations

1. Interpolate Euler boundary conditions from PM

$$
(\omega, \delta, \rho)_{E \mid b c}^{k}=\operatorname{Interp}(\omega, \delta, \rho)_{P M}^{k}
$$

2. Solve Euler domain subject to the above BCs to obtain the updated Euler solution: $\vec{S}_{E}^{k}$

3. Convert Euler solution to particles:

$$
\vec{S}_{E}^{k} \rightarrow(\omega, \delta, \rho)_{E}^{k}
$$

4. Project Euler solution onto PM to obtain: $(\omega, \delta, \rho)_{P M}^{k+1}=(\omega, \delta, \rho)_{P M}^{d i f}+\operatorname{Proj}(\omega, \delta, \rho)_{E}^{k}$

5. Repeat until convergence is met:

$$
\left\|(\omega, \delta, \rho)_{P M}^{k+1}-(\omega, \delta, \rho)_{P M}^{k}\right\|<\varepsilon_{c}
$$

Step 2: Perform CVPM algorithm (Section 2.6). Use new particle solution as starting point for next step (return to Step 0)

Without this type of iterative technique, it would be necessary to solve the Hamilton-Jacobi equation subject to both external boundary conditions located along the PM boundary, in addition to internal boundary conditions placed at the airfoil 
surface and at shock locations. Specifically, it was found to be unpractical to implement the internal airfoil boundary conditions. This involves the approximation of the curved airfoil surface as discontinuities in the scalar potential field, $\phi$. However, in order to resolve these discontinuity strengths, an extremely fine PM density was required (or a body-fitted which would cancel the high computational efficiency of the PM method), thus making it a computationally inefficient approach. The CVPM algorithm above is implemented in order to remove the need for these internal boundary conditions and allow the PM and Euler solutions to converge together. It is important to note that whenever the PM solution is modified by the Euler solver, it is necessary to recalculate the PM velocities.

\subsubsection{Euler Solution Integration}

In order to obtain vorticity, dilatation and density from the Euler domain, the Euler solution must be integrated. This integration procedure transforms the cell-centered Euler solution into a set of temporary "particles", which may then be projected onto the PM. This essentially amounts to the assignment of circulation, divergence, mass and volume across the Euler domain. Firstly, we obtain circulation and divergence of an Euler cell with the use of Kelvin-Stokes' theorem and Gauss' theorem, respectively:

$$
\begin{aligned}
\Gamma & =\iint_{A}(\nabla \times \vec{u}) d A=\oint_{s} \vec{u} \cdot \vec{\tau} d S \\
\Delta & =\iint_{A}(\nabla \cdot \vec{u}) d A=\oint_{s} \vec{u} \cdot \vec{n} d S
\end{aligned}
$$


Here, $A$ is the cell area, $S$ the cell boundary and $\vec{\tau}$ and $\vec{n}$ the boundary tangential and normal vectors respectively. The geometry for this integration is shown in Figure 4.3. The Euler solution is given at cell centres labeled $1,2,3$ and 4 . The integration results in an Euler "particle" created at the Euler grid node central to the 4 adjacent FV cells.
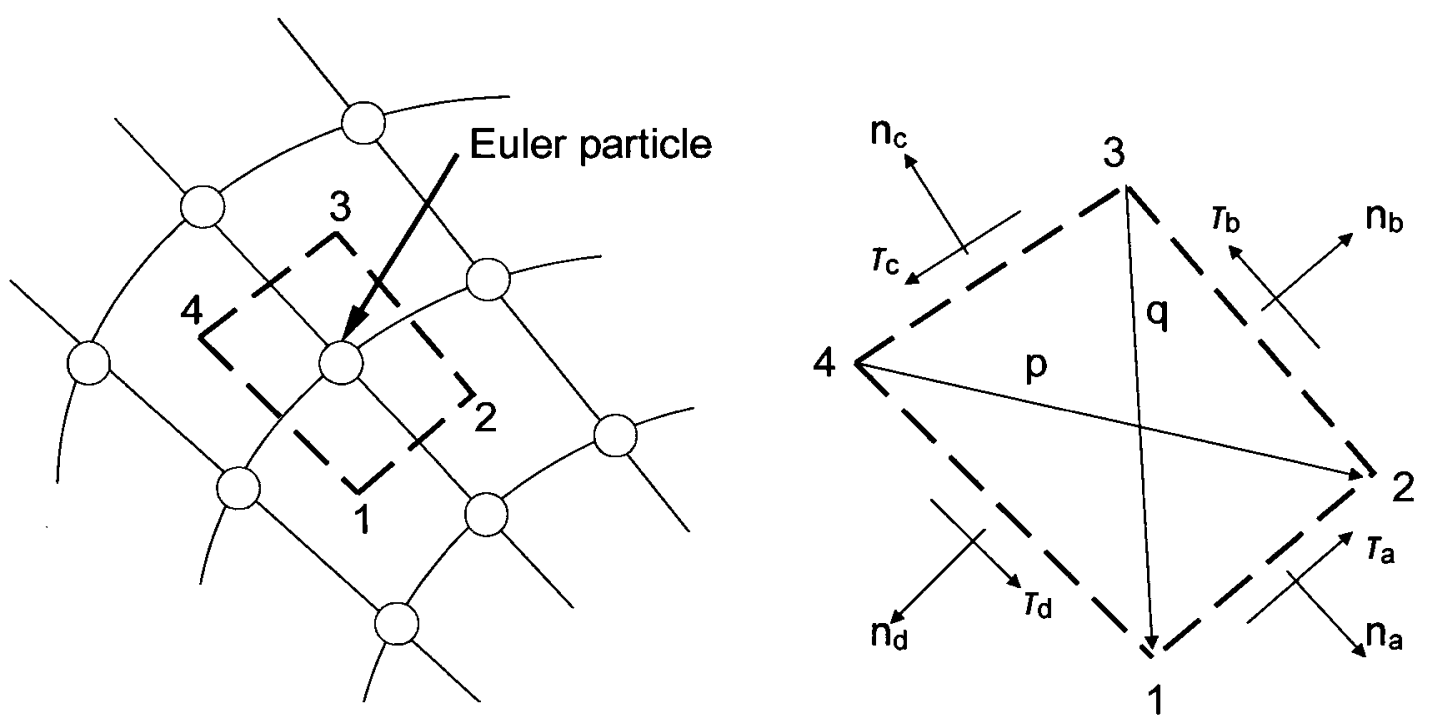

Figure 4.3: Cell geometry for the integration of the Euler solution into a particle field.

In this way, the circulation and divergence are written as a discrete summation:

$$
\begin{aligned}
\Gamma & =\sum_{i=a}^{d} S_{i}\left(\vec{u}_{i} \cdot \vec{\tau}_{i}\right) \\
\Delta & =\sum_{i=a}^{d} S_{i}\left(\vec{u}_{i} \cdot \vec{n}_{i}\right)
\end{aligned}
$$


where $S_{i}$ is the length of the $i^{\text {th }}$ side. In general, the swept area of this integral procedure traces a quadrilateral shape, whose volume, $V$, is computed with the quadrilateral cross-diagonal vectors $\vec{p}$ and $\vec{q}$ as follows:

$$
V=\frac{1}{2}|\vec{p} \times \vec{q}|
$$

Mass is simply obtained using the average of the surrounding cell densities and cell volume:

$$
M=\frac{1}{4}\left(\rho_{1}+\rho_{2}+\rho_{3}+\rho_{4}\right) V
$$

It is important to consider the compatibility between the Euler and PM grids. The purpose of this integral procedure is to obtain information from the Euler solution which is to be projected onto and replace the values on the PM grid. In order to ensure this smooth projection, the Euler grid density must be no larger than the PM grid density. This is not an issue near the airfoil surface where the Euler grid is fine. However, as one travels away from the airfoil, the Euler grid typically coarsens and the solution must be adjusted. The procedure used is to implement a finite-element 2-D linear interpolation, resulting in a denser Euler "particle" distribution where required in the Euler solution. The geometry for this interpolation is shown in Figure 4.4 where $(x, y)$ is the physical domain and $(\xi, \eta)$ is the computational domain:

This interpolation procedure for a given quantity, $\alpha$, is written in discrete form 


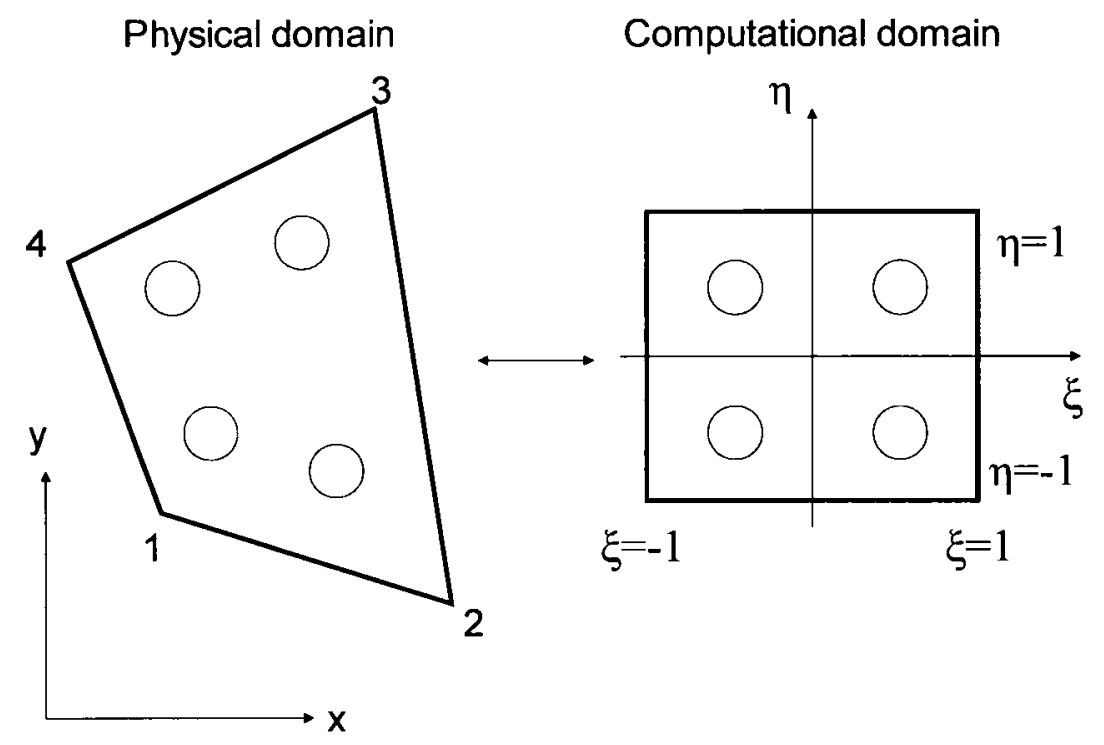

Figure 4.4: Transformation geometry for cell finite-element 2-D linear interpolation.

as:

$$
\alpha(\xi, \eta)=\frac{1}{4} \sum_{k=1}^{4} \alpha_{k}\left(1+\xi \xi_{k}\right)\left(1+\eta \eta_{k}\right)
$$

and results in uniform compatibility between the PM and Euler grids across their entire overlap.

\subsection{Non-dimensional Hamilton-Jacobi Equation}

The Hamilton-Jacobi equation (2.37), derived in Section 2.3, is written in dimensional form. In order to ensure compatibility between the Euler solver and the CVPM, it is necessary to use the same non-dimensionalization scheme for the Hamilton-Jacobi equation as with the Euler solver formulation. With reference to the scheme applied in 
the Euler solver in Section 3.1, the Hamilton-Jacobi variables are non-dimensionalized according to:

$$
\begin{array}{ll}
\phi=\frac{\phi^{*}}{V_{\infty}^{*} L^{*}} & \vec{w}=\frac{\vec{w}^{*}}{V_{\infty}^{*}} \quad t=\frac{V_{\infty}^{*} t^{*}}{L^{*}} \\
p=\frac{p^{*}}{\rho^{*} V_{\infty}^{* 2}} & \rho=\frac{\rho_{\infty}^{*}}{\rho_{\infty}^{*}} \quad q=\frac{q^{*}}{V_{\infty}^{* 2}}
\end{array}
$$

which leads to a non-dimensional equation given as:

$$
\frac{\partial \phi}{\partial t}+\frac{1}{2} \nabla \phi^{2}+\vec{w} \cdot \nabla \phi=q-\frac{1}{(\gamma-1) M^{2}} \rho^{\gamma-1}-\frac{1}{2} \vec{w}^{2}
$$

\subsection{Non-Reflective Boundary Conditions}

The artificial truncation of the domain requires special treatment at the PM boundaries. In compressible flow, it is necessary to impose absorbing boundary conditions which do not allow numerical reflection of dilatation (or density) oscillations back into the solution domain. This procedure is a two-pronged approach, modified for

the present work based on a method proposed by Freunde [55], which features the addition of an artificial convection term in the Hamilton-Jacobi equation which tends to support the propagation of disturbances, while a damping function is implemented on the dilatation, which drives the solution to a quiescent state away from the domain of interest (the airfoil in this case).

With the addition of the artificial convective velocity, $U_{c}$, the Hamilton-Jacobi equation can be written as:

$$
\frac{\partial \phi}{\partial t}+U_{c}(r) \frac{\partial \phi}{\partial r}+H(\nabla \phi)+\vec{w} \cdot \nabla \phi=f
$$


where $U_{c}$ has the form

$$
U_{c}(r)=\frac{1}{2} U_{\max }\left[1+\tanh \left(-F\left(r_{\max }-r\right)\right)\right]
$$

where $U_{\max }, F$ and $r_{\max }$ are specified constants. Dilatation oscillations are damped according to:

$$
\delta(r)=\delta(r) \zeta(r)
$$

$\zeta(r)$ is a Gaussian damping function given by:

$$
\zeta=\left\{\begin{array}{ll}
e^{-\sigma\left(r-r_{\max }\right)^{2}}, & \text { if } \left.r>r_{\max }\right) \\
1 & \text { else }
\end{array}\right\}
$$

where $\sigma$ is also a specified constant. Figure 4.5 demonstrates the geometry required to implement these conditions. 


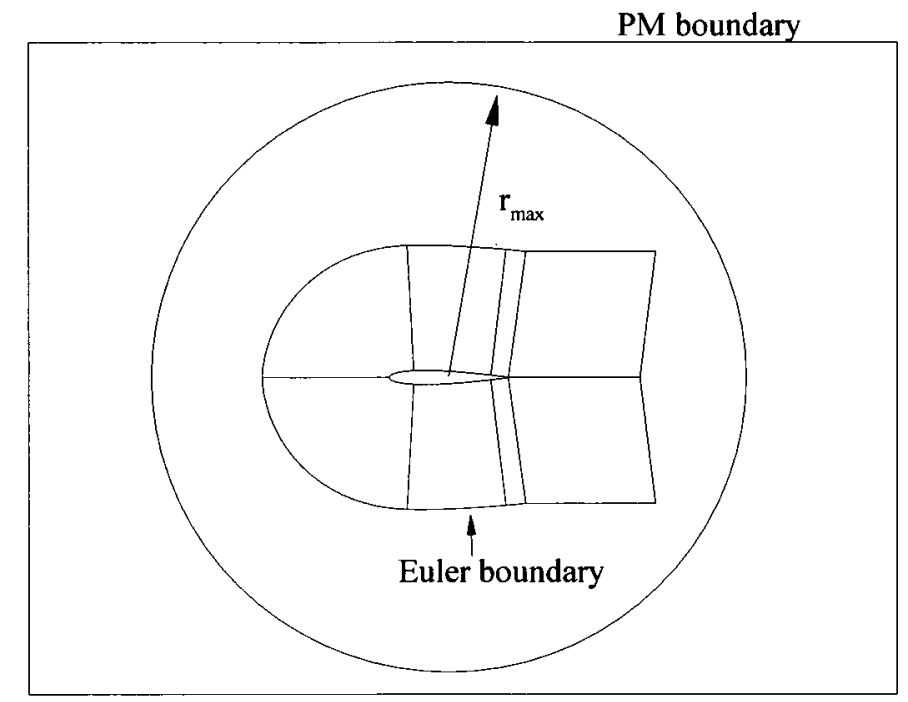

Side view

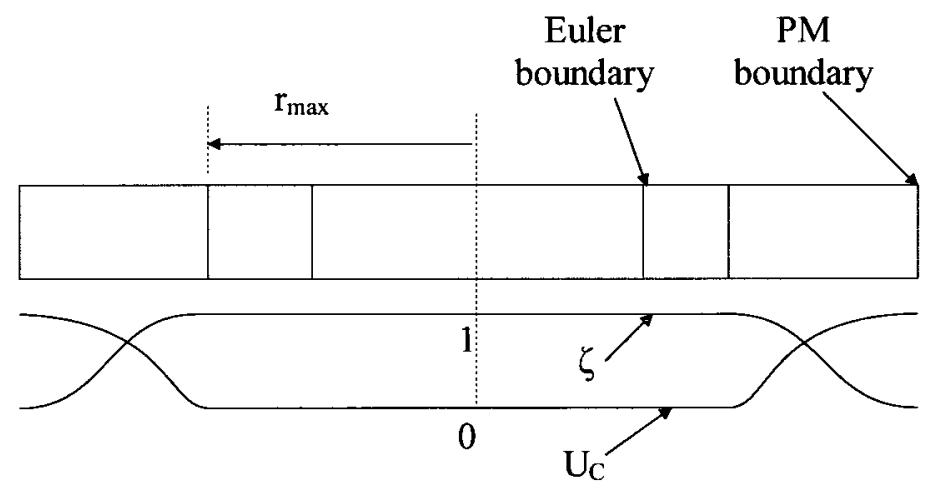

Top view

Figure 4.5: Side and top view of the hybrid model including the newly developed non-reflective boundary condition. The top view demonstrates the radial variation of artificial convective velocity and dilatation cut-off functions. 
The effect of the non-reflective BCs is evident in Figure 4.6, which displays the time history of kinetic energy $\left(\frac{1}{2} \rho_{i j}\left|u_{i j}\right|^{2}\right)$ on the PM.

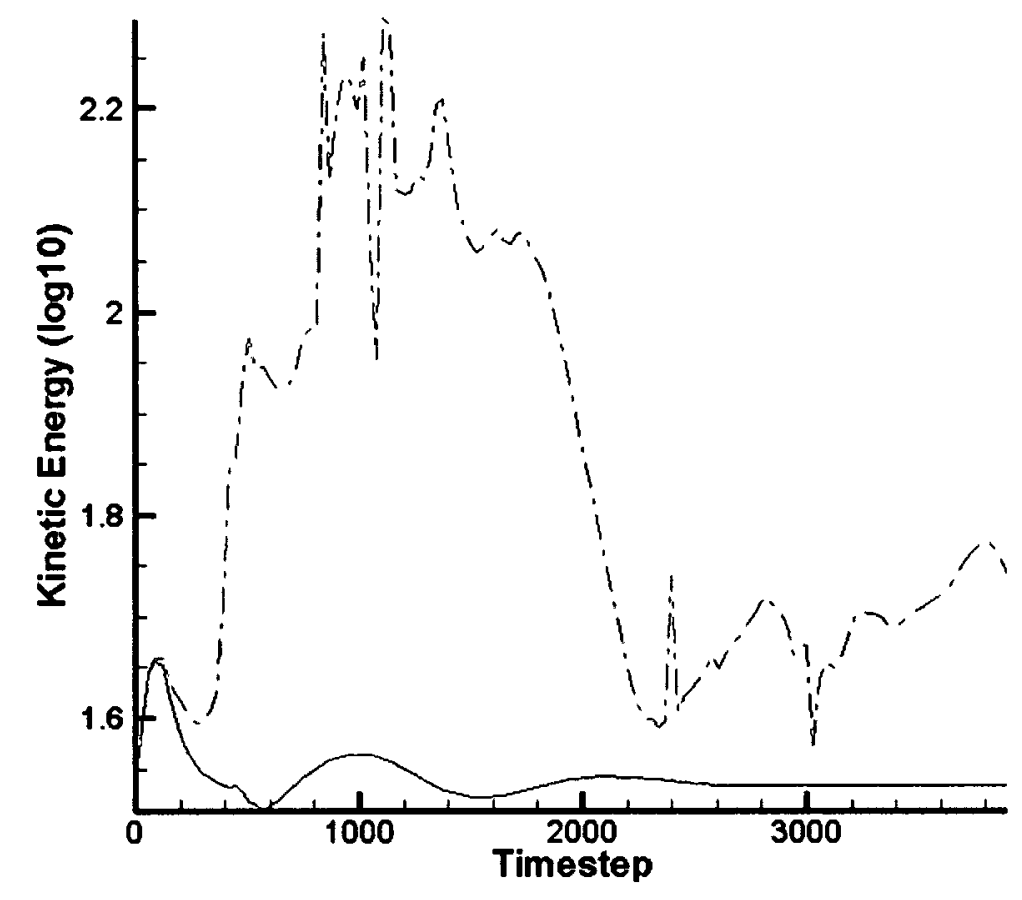

Figure 4.6: Time history of particle mesh kinetic energy for a impulsively pitched NACA 0012 airfoil with non-reflective boundary conditions (solid line) and without the non-reflective condition (dashed line).

Results are compared for a NACA 0012 airfoil which is impulsively pitched through 1 cycle at $\mathrm{t}=0 \mathrm{~s}$ and the flow is allowed to approach a steady state. Experience has shown that the outgoing waves are absorbed effectively with constants $U_{\max }=-0.25$, $F=0.1$ and $\sigma=0.2$. The $r_{\max }$ extent is set beyond the boundary locations for the Euler solver which in this case can be $2.25 \mathrm{c}$.

It is seen that without the non-reflective BCs, the dilatation waves are reflecting 
back into the domain in an un-physical fashion, further complicating the flow and certainly not allowing it to approach a steady state. However, with the non-reflecting conditions implemented, it is seen that the dilatations waves are not allowed to reenter the computational domain, thereby allowing the flow to smoothly settle back to the steady condition. This phenomenon is illustrated with the dilatation contours in Figure 4.7.

Without non-reflect BCs

$$
n=1
$$

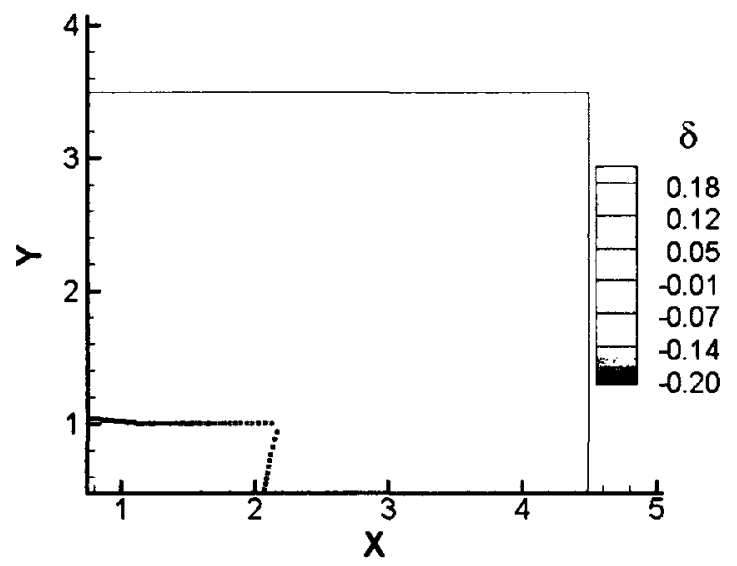

With non-reflective $\mathrm{BCs}$

$$
n=1
$$

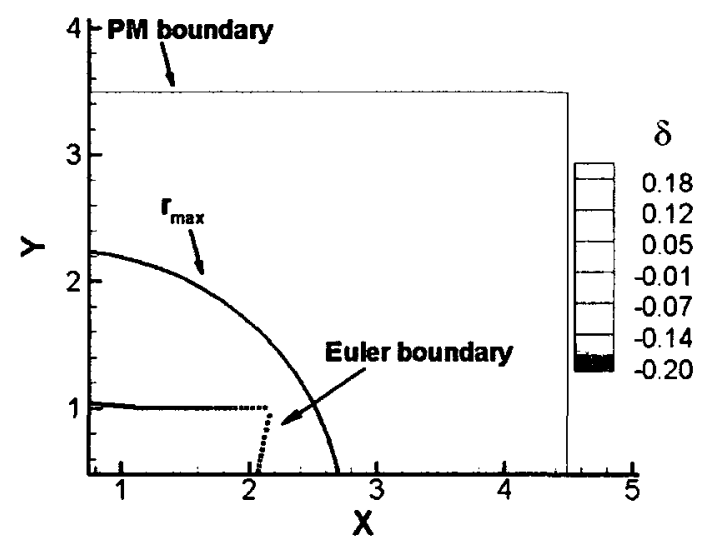

Figure 4.7: Dilatation contours at three timesteps with and without non-reflective boundary conditions implemented. Sampling timesteps are $n=1,11,14$ for a NACA 0012 airfoil in impulsive pitch at $\mathrm{M}=0.5$. 


$$
n=11
$$

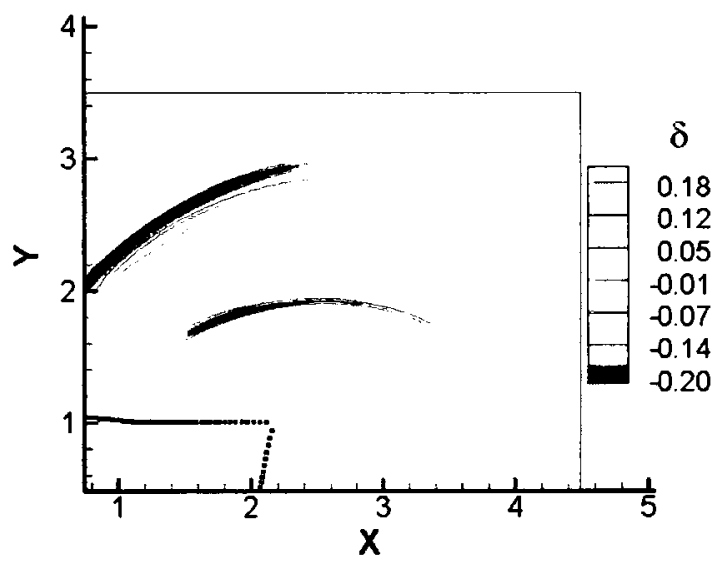

$$
n=14
$$

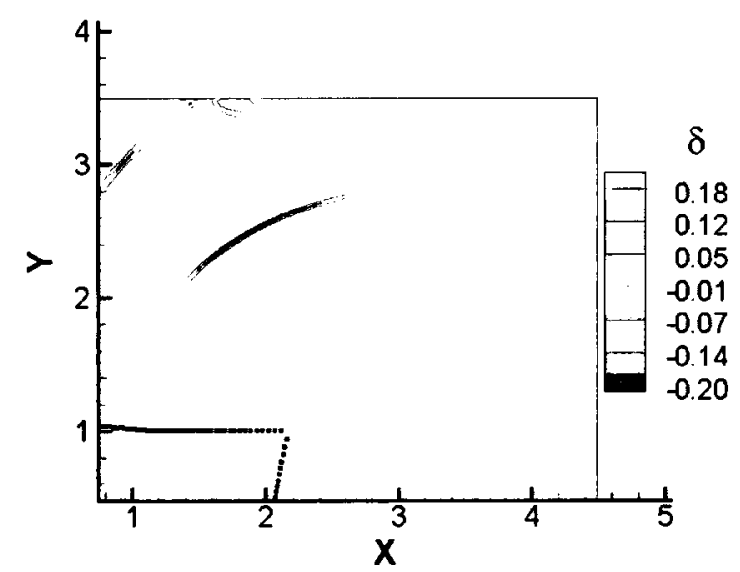

$n=11$

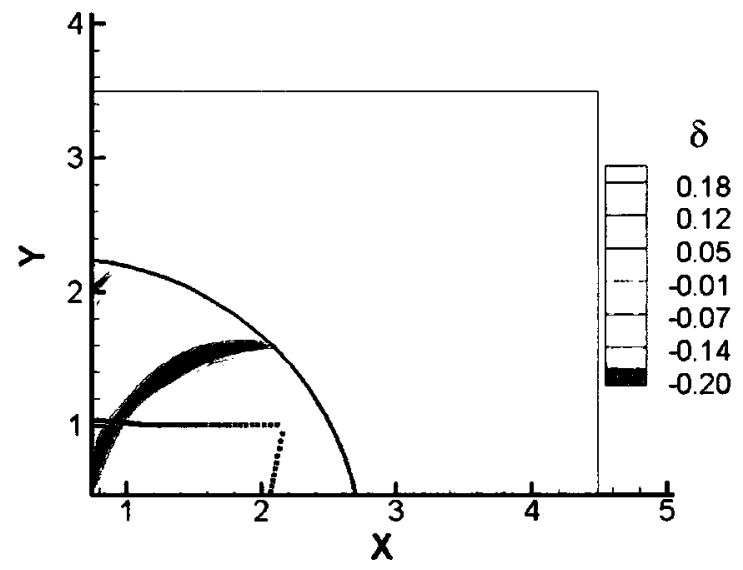

$n=14$

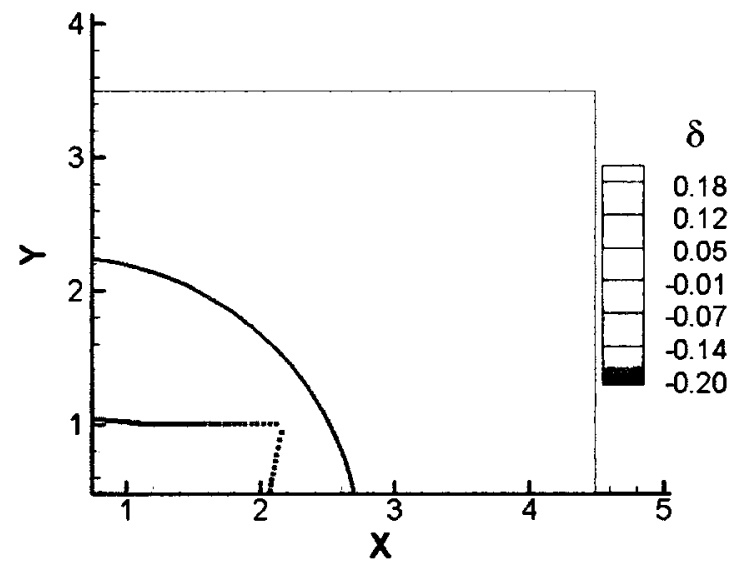

Figure 4.7: Dilatation contours at three timesteps with and without non-reflective boundary conditions implemented. Sampling timesteps are $n=1,11,14$ for a NACA 0012 airfoil in impulsive pitch at $\mathrm{M}=0.5$. (cont) 


\section{Chapter 5}

\section{Verification and Validation}

In this section, verification and validation results are presented for the newly developed Hybrid Euler-Particle Method (HEPM) code. All computations have been performed on the Rotorcraft Research Group's (RRG) Beowulf cluster using a single Genuine Intel Pentium $3.2 \mathrm{GHz}$ processor with i386 architecture and 1GB of RAM.

The chapter is divided into 3 main topics: 1) verification of the numerical method, 2) validation with experimental unsteady flows and 3) airfoil-vortex interaction (AVI) tests.

For the verification tests, the aim is to verify that the new numerical method, which is time-accurate in nature, ultimately converges in time to a valid steadystate solution. This has been tested by simulating a NACA 0012 airfoil at both low and high Mach (Ma) numbers, designated as STEADY1 and STEADY2 tests. In addition, an unsteady test for a rapidly pitching airfoil, designated as UNSTEADY1, was performed to check the continuity of particle mesh (PM) quantities, i.e. $\omega$ and 
$\delta$, across the Euler-PM boundary, as well as to check the preservation of the wake vorticity in comparison with the numerically dissipative full Euler solutions. Rapid pitch type motion is selected in this case in order to be able to look closely at the wake structure before it exits the PM domain.

For the unsteady validation tests, the aim was to verify that the solver can converge to a periodic unsteady solution in time. Validation to experiment has been performed in comparison to the Advisory Group for Aerospace Research and Development (AGARD) test results. Two validation cases are considered: a sinusoidally pitching and a ramp pitching NACA0012 airfoil designated as the UNSTEADY2 and UNSTEADY3 tests, respectively.

Lastly, the AVI problem so typical for rotorcraft is examined at various flow parameters in AVI1 and AVI2.

\subsection{Verification}

\subsubsection{Steady Flow Convergence}

The steady flow convergence behaviour of the method was examined at low and high freestream Mach numbers. Table 5.1 specifies the code input parameters for STEADY1. Note that in all input parameter tables " $\varepsilon_{E}$ " is the convergence criterion for the Euler solver, "CFL" refers to the Courant-Friedrichs-Levy condition and "PM $\Delta h "$ is the particle mesh spacing.

Figures 5.1-5.3 clearly show that steady conditions are indeed achieved. Results 
Table 5.1: HEPM input parameters: STEADY1

\begin{tabular}{ll|ll|ll}
\hline \hline General & & Euler solver & & Particle method & \\
\hline Airfoil & N0012 & Grid density & 20000 cells & $X_{\min , \max }$ & $-3.5,4.5$ \\
Chord & $0.1016 \mathrm{~mm}$ & Extent & 1 chord & $Y_{\min , \max }$ & $-2.5,2.5$ \\
Mach & 0.40 & Implicit CFL & 105 & PM $\Delta h$ & 0.02 \\
Motion & steady & Steady $\varepsilon_{E}$ & $1 \mathrm{E}-6$ & Particles/cell & 4 \\
$\Delta t$ & 0.007 & Unsteady $\varepsilon_{E}$ & $1 \mathrm{E}-3$ & Project/Interp & TSC \\
\hline \hline
\end{tabular}

are presented for the Euler domain only. For this relatively small Mach number $(M a=0.4)$, it is seen that density and dilatation perturbations do not extend a long distance from the airfoil. With reference to Figure 5.3, it is important to note a thin stream of vorticity emanating from the airfoil trailing edge. The appearance of this vorticity is unphysical, as there is no theoretical means of vorticity creation for an inviscid and steady simulation. It has been determined that this vorticity is a numerical error due to the nature of the numerical method implemented in the Euler domain. The Euler solver is a cell-centered finite-volume formulation, meaning that boundary conditions are not enforced exactly at the solid surface, but a small distance away at the centre of the cell adjacent to the surface. As a result, the Kutta condition, requiring the flow from the upper and lower surfaces of the airfoil to emanate from the trailing edge parallel to one another, cannot be implemented exactly, causing a small difference in the flow velocity between the upper and lower trailing edge surface, which in turn creates the erroneous vorticity. It is noted though that this vorticity is small at less than $3 \%$ of typical rotorcraft wake vortex strengths. 


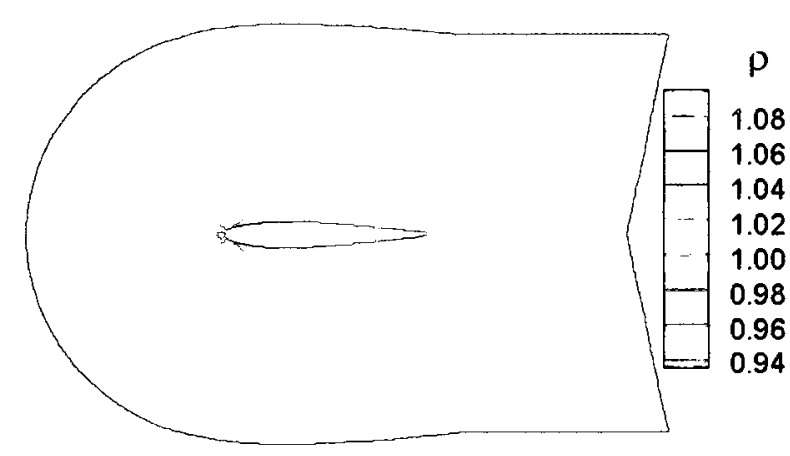

(a)

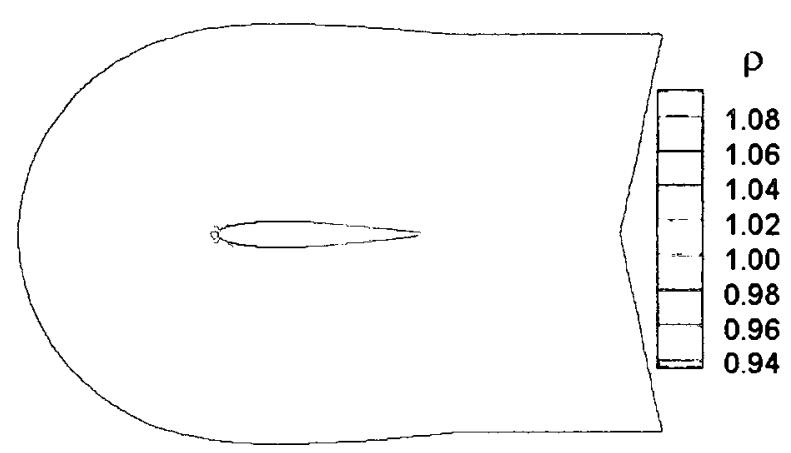

(b)

Figure 5.1: Density contours at (a) $n=1$ and (b) $n=1000$ timesteps for the STEADY1 case.

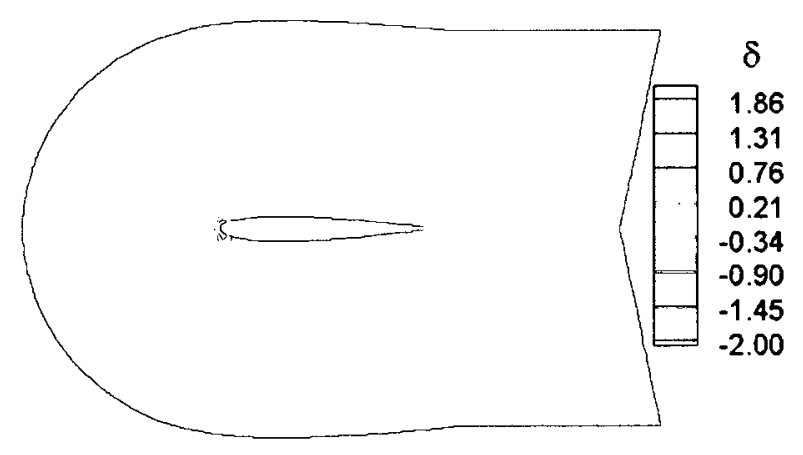

(a)

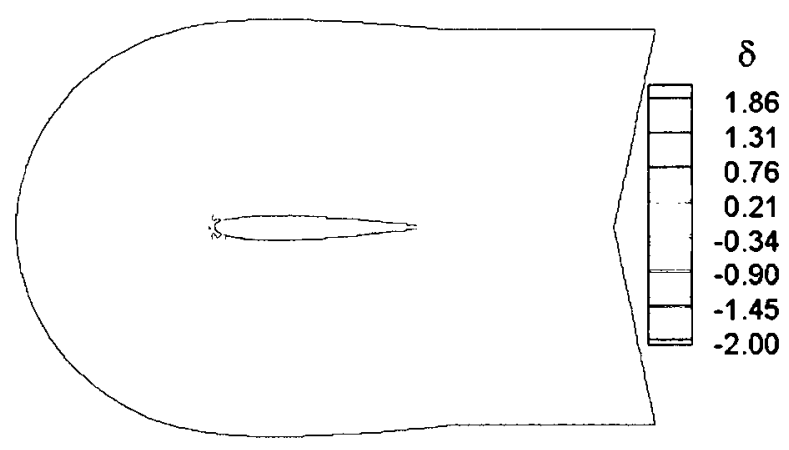

(b)

Figure 5.2: Dilatation contours at (a) $n=1$ and (b) $n=1000$ timesteps for the STEADY1 case. 


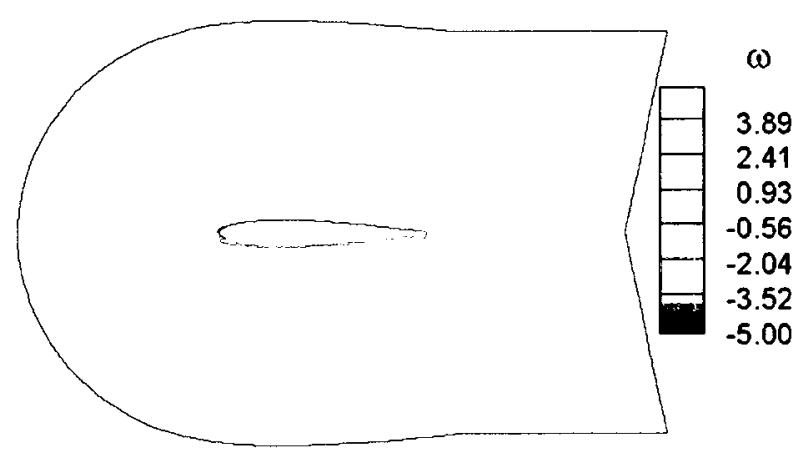

(a)

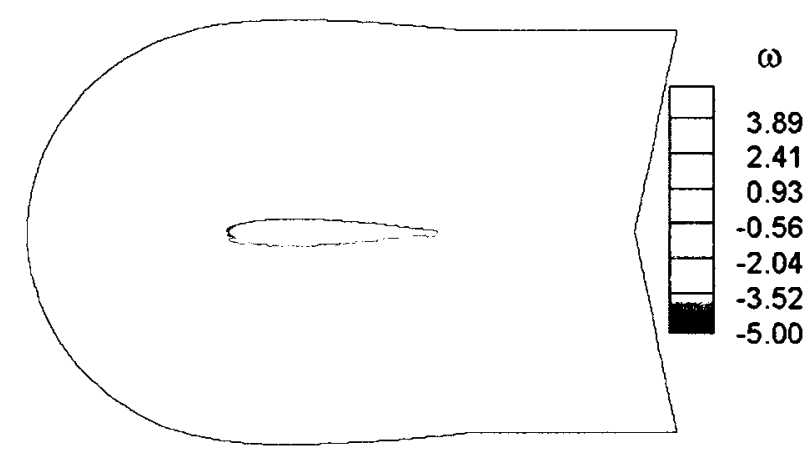

(b)

Figure 5.3: Vorticity contours at (a) $n=1$ and (b) $n=1000$ timesteps for the STEADY1 case.

Table 5.2 specifies code input parameters for STEADY2.

Table 5.2: HEPM input parameters: STEADY2

\begin{tabular}{ll|ll|ll}
\hline \hline General & & Euler solver & & \multicolumn{2}{|l}{ Particle method } \\
\hline Airfoil & N0012 & Grid density & 20000 cells & $X_{\min , \max }$ & $-3.5,4.5$ \\
Chord & $0.1016 \mathrm{~mm}$ & Extent & 1 chord & $Y_{\min , \max }$ & $-2.5,2.5$ \\
Mach & 0.755 & Implicit CFL & 50 & PM $\Delta h$ & 0.02 \\
Motion & steady & Steady $\varepsilon_{E}$ & $1 \mathrm{E}-6$ & Particles/cell & 4 \\
$\Delta t$ & 0.007 & Unsteady $\varepsilon_{E}$ & $1 \mathrm{E}-3$ & Project/Interp & TSC \\
\hline \hline
\end{tabular}

Again, Figures 5.4-5.6 clearly show that steady conditions are achieved. This higher Mach number $(M a=0.755)$ case is a transonic case. The density and dilatation contours indicate the presence of a shock wave extending 0.5 chord lengths from the airfoil surface. 


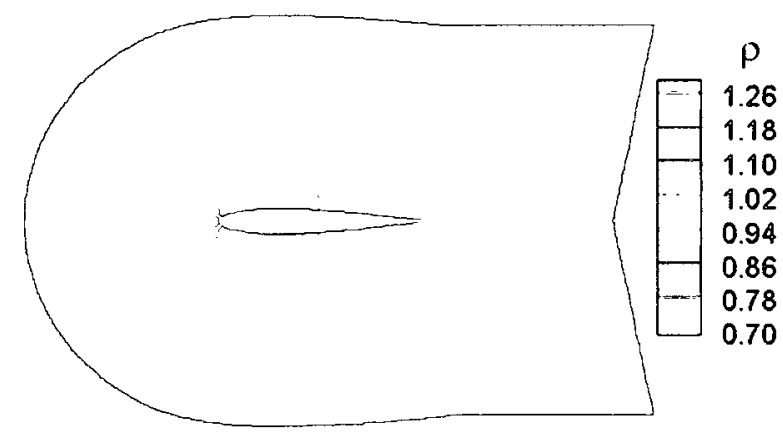

(a)

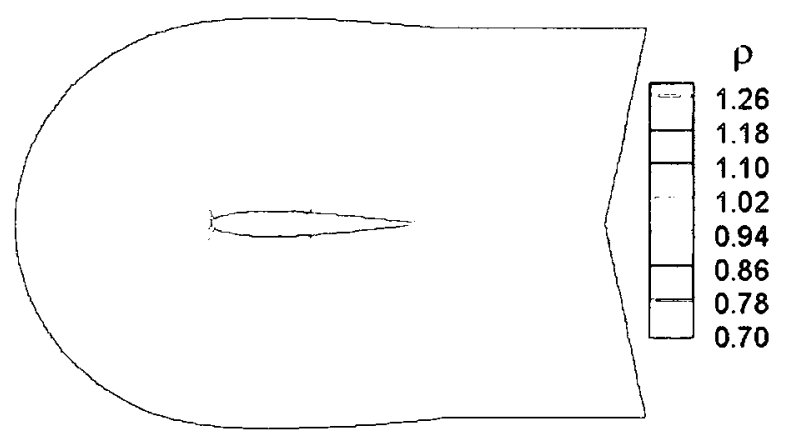

(b)

Figure 5.4: Density contours at (a) $n=1$ and (b) $n=1000$ timesteps for the STEADY2 case.

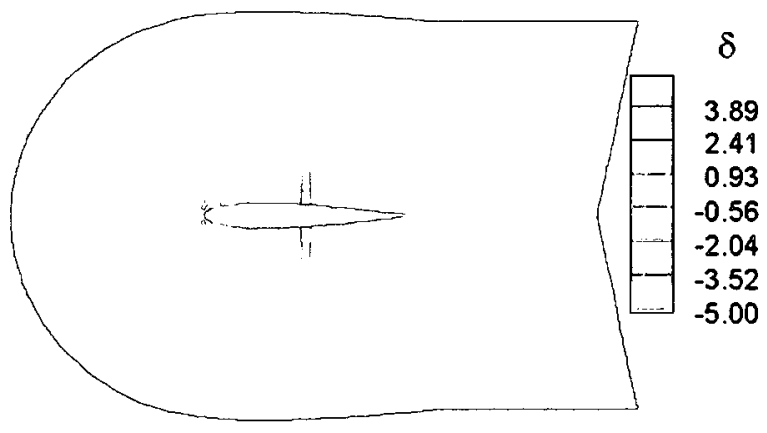

(a)

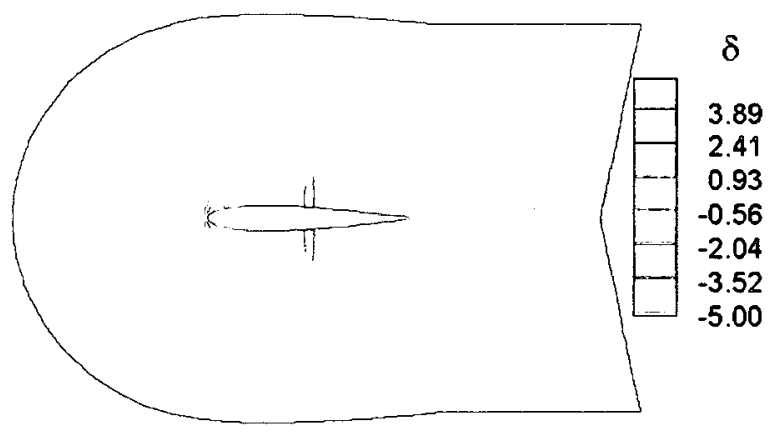

(b)

Figure 5.5: Dilatation contours at (a) $n=1$ and (b) $n=1000$ timesteps for the STEADY2 case. 


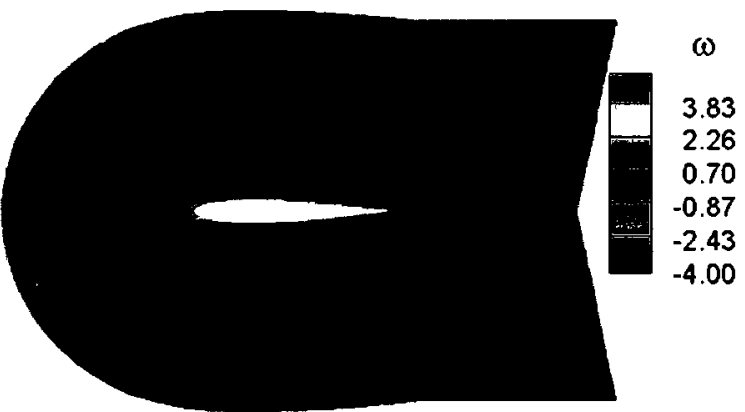

(a)

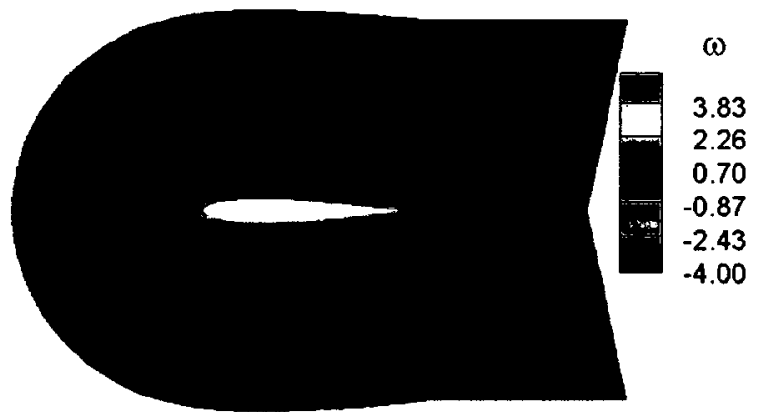

(b)

Figure 5.6: Vorticity contours at (a) $n=1$ and (b) $n=1000$ timesteps for the STEADY2 case.

Figure 5.7 further demonstrates the steady convergence for both flow speeds. For $\alpha=0$ it is expected that the pressure coefficient, $C_{p}$, is symmetrical between the top and bottom surfaces of the airfoil and remains constant over time. This is clearly shown in Figure 5.7, including steady shock capturing for the STEADY2 case. 


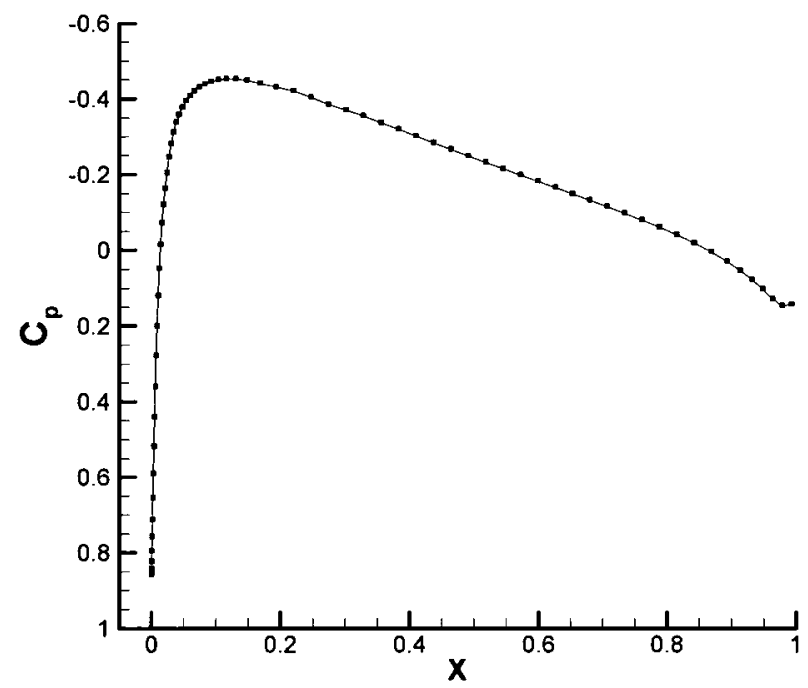

(a)

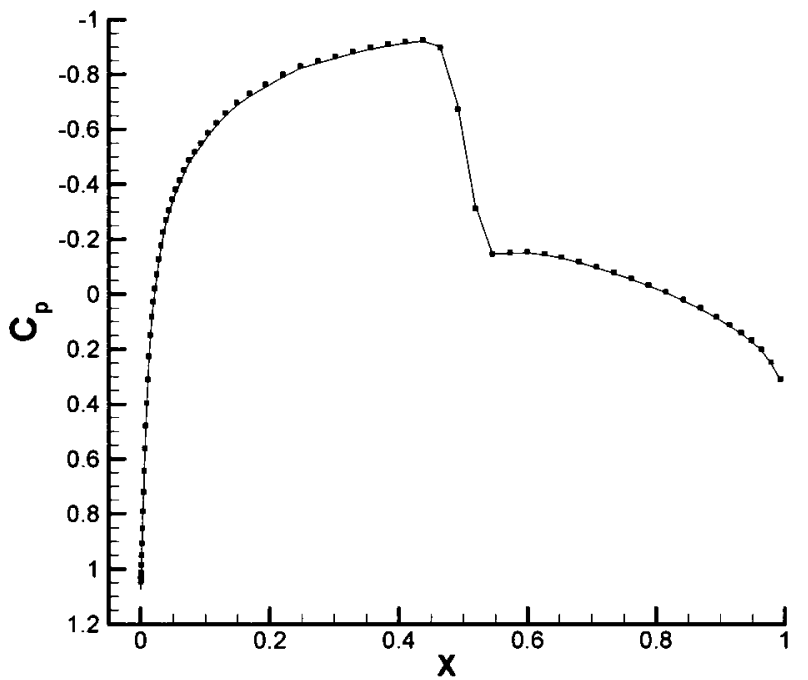

(b)

Figure 5.7: Chordwise $C_{p}$ distribution for $\mathrm{n}=1$ (solid line) and $\mathrm{n}=1000$ (square) timesteps for (a) STEADY1 and (b) STEADY2 cases 


\subsubsection{Rapidly Pitching Airfoil}

An initial convergence study for unsteady flow was done for a rapidly pitching NACA 0012 airfoil at a moderate Mach number $(M a=0.60)$. Table 5.3 presents the simulation parameters for this case. As previously stated, the extremely rapid pitching motion of the airfoil was selected in order to allow enough detail of the vortex wake structure to remain inside the PM region.

Table 5.3: HEPM input parameters: UNSTEADY1

\begin{tabular}{ll|ll|ll}
\hline \hline General & & Euler solver & & Particle method & \\
\hline Airfoil & N0012 & Grid density & 20000 cells & $X_{\min , \max }$ & $-3.5,4.5$ \\
Chord & $0.1016 \mathrm{~mm}$ & Extent & 1 chord & $Y_{\min , \max }$ & $-2.5,2.5$ \\
Mach & 0.60 & Implicit CFL & 80 & PM $\Delta h$ & 0.02 \\
Motion & sinusoidal & Steady $\varepsilon_{E}$ & $1 \mathrm{E}-6$ & Particles/cell & 4 \\
$\Delta t$ & 0.007 & Unsteady $\varepsilon_{E}$ & $1 \mathrm{E}-3$ & Project/Interp & TSC \\
\hline \hline
\end{tabular}

UNSTEADY1 is a sinusoidal pitch case about $0.25 \mathrm{c}$ according to the equation $\alpha=$ $\alpha_{m}+\alpha_{0} \sin 2 k t$. Table 5.4 displays the more detailed motion parameters implemented where $k$ is reduced frequency.

Table 5.4: Motion parameters for UNSTEADY1

\begin{tabular}{c|c|c|c|c}
\hline \hline $\mathrm{M}$ & $\begin{array}{c}\alpha_{m} \\
(\text { deg. })\end{array}$ & $\begin{array}{c}\alpha_{0} \\
(\text { deg. })\end{array}$ & $\begin{array}{c}\mathrm{f} \\
(\mathrm{Hz})\end{array}$ & $\mathrm{k}$ \\
\hline 0.6 & 0.0 & 2.0 & 3071 & 4 \\
\hline \hline
\end{tabular}

\section{Lift Hysteresis Convergence}

Figure 5.8 shows the convergence of the lift hysteresis. It is found that the results converge after 6 cycles. Normally, with a typical Euler solver, it is found that only 
3 cycles are expected for lift convergence. It is determined that it takes a bit longer for HEPM due to the extremely aggressive nature of the pitch speed, required for an in-depth look at the vortex wake structure before it exits the PM domain. Figure

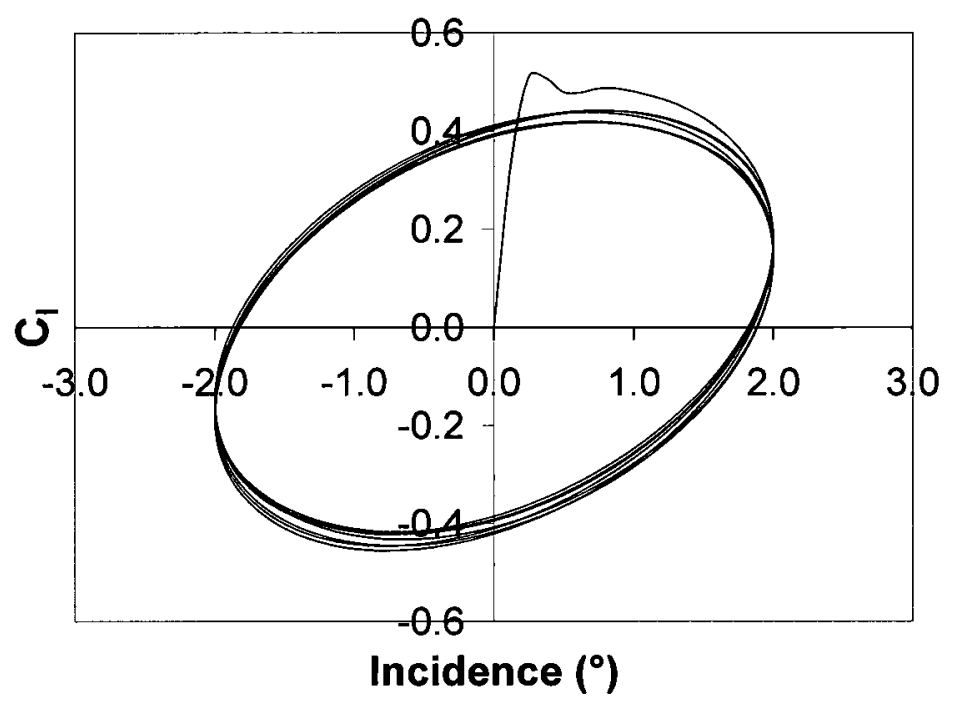

Figure 5.8: Lift hysteresis convergence history for the UNSTEADY1 case.

5.9 presents a comparison of the converged lift hysteresis loop between the reference Euler solution and HEPM for UNSTEADY1. They are found to be in good agreement. Figure 5.10 provides the lift time history for the reference Euler and HEPM solutions. It is verified that there is no time shift between the results. 


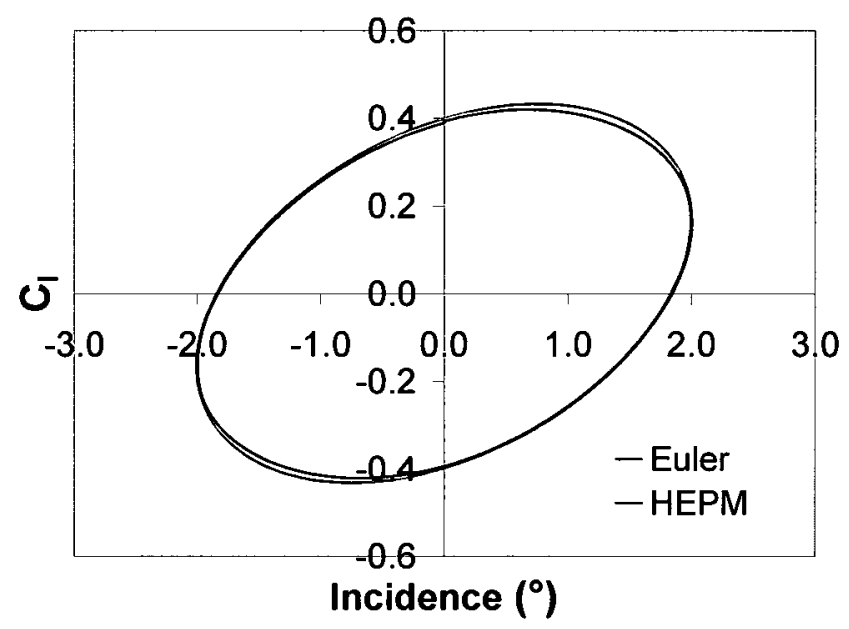

Figure 5.9: Lift hysteresis comparison between Euler reference solution and HEPM for the UNSTEADY1 case. Results shown for the 3rd cycle.

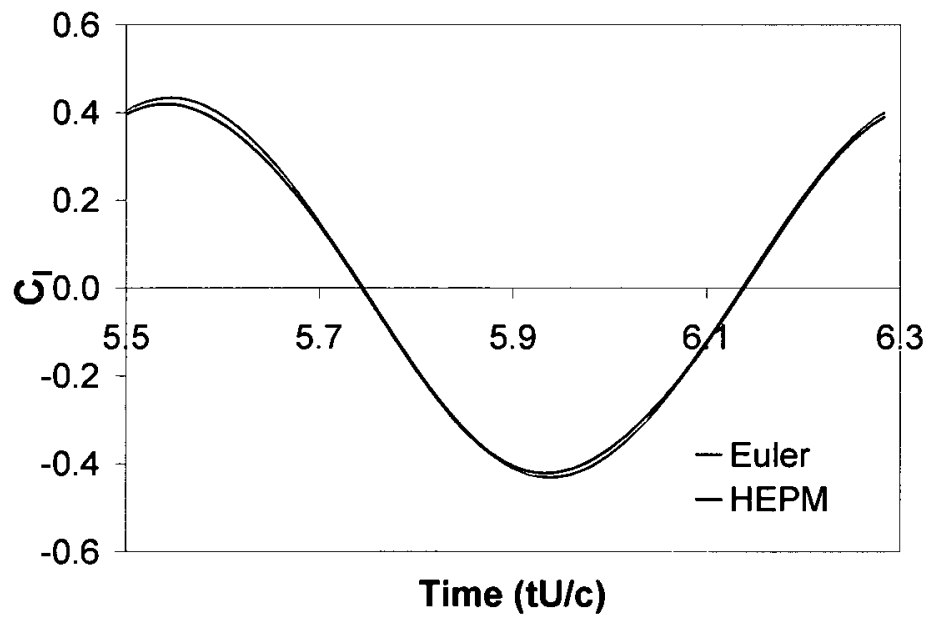

Figure 5.10: Lift history comparison between Euler reference solution and HEPM for the UNSTEADY1 case. Results shown for the 3rd cycle. 


\section{Variable Continuity}

An examination of the vorticity and dilatation contours in Figure 5.11 reveal a smooth transition between the Euler and CVPM domains for the UNSTEADY1 case. This assures us that the coupling algorithm between the two methods is working properly. If one were to notice discontinuities in vorticity and dilatation across these interfaces, it would be due to the iterative coupling scheme, outlined in Section 4.2, failing to converge completely to the desired convergence criterion. In this type of nonconvergence situation it can be said that the Euler solution is not compatible to the PM solution, and thus smooth transition problems will begin to occur, which will subsequently result in waves being introduced through the Hamilton-Jacobi equation.

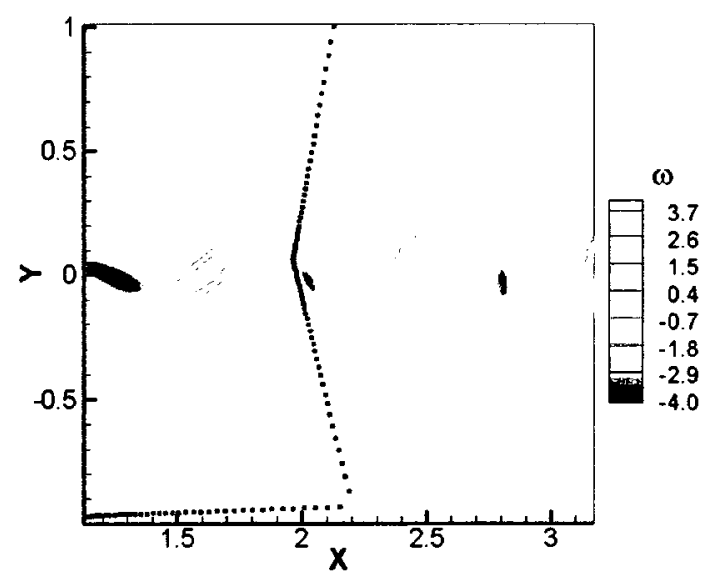

(a)

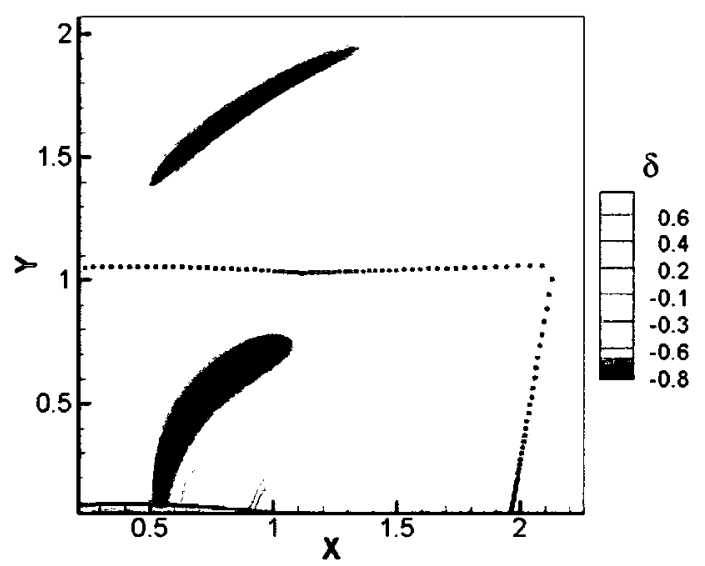

(b)

Figure 5.11: (a) Vorticity and (b) dilatation contours as they cross the interface (squares) of the overlap between the two methods for the UNSTEADY1 case. 


\section{Vorticity Preservation}

One of the main drawbacks of grid-based numerical methods, as identified in the literature survey, is the unphysical numerical dissipation of vorticity due to the discretization of the convective terms in the governing equations. To prove the effectiveness of HEPM in eliminating this issue, we will examine the dissipation of a vortex generated as a result of a rapidly pitching motion, corresponding to the UNSTEADY1 case. We will first demonstrate the problems encountered with the full Euler solver (CMB). As shown in Figure 5.12, the region of interest was selected to be located in the airfoil wake centered at $2.25 \mathrm{c}$ downstream of the trailing edge. Four grids, displayed in Figure 5.13, were used in the analysis: coarse, medium, fine and extra fine. The associated grid densities and required CPU times (for the Euler simulations only) are found in Table 5.5.

Table 5.5: Euler grid densities and runtimes for vorticity preservation investigation

\begin{tabular}{l|l|l|l}
\hline \hline Grid Type & Total Nodes & Downstream Block Nodes & CPU time \\
\hline Coarse & 11,660 & 4,400 & $11 \mathrm{~min}$ \\
Medium & 32,120 & 17,600 & $31 \mathrm{~min}$ \\
Fine & 99,440 & 70,400 & $1 \mathrm{hr} 40 \mathrm{~min}$ \\
Extra fine & 169,840 & 140,800 & $3 \mathrm{hr} 10 \mathrm{~min}$ \\
\hline \hline
\end{tabular}




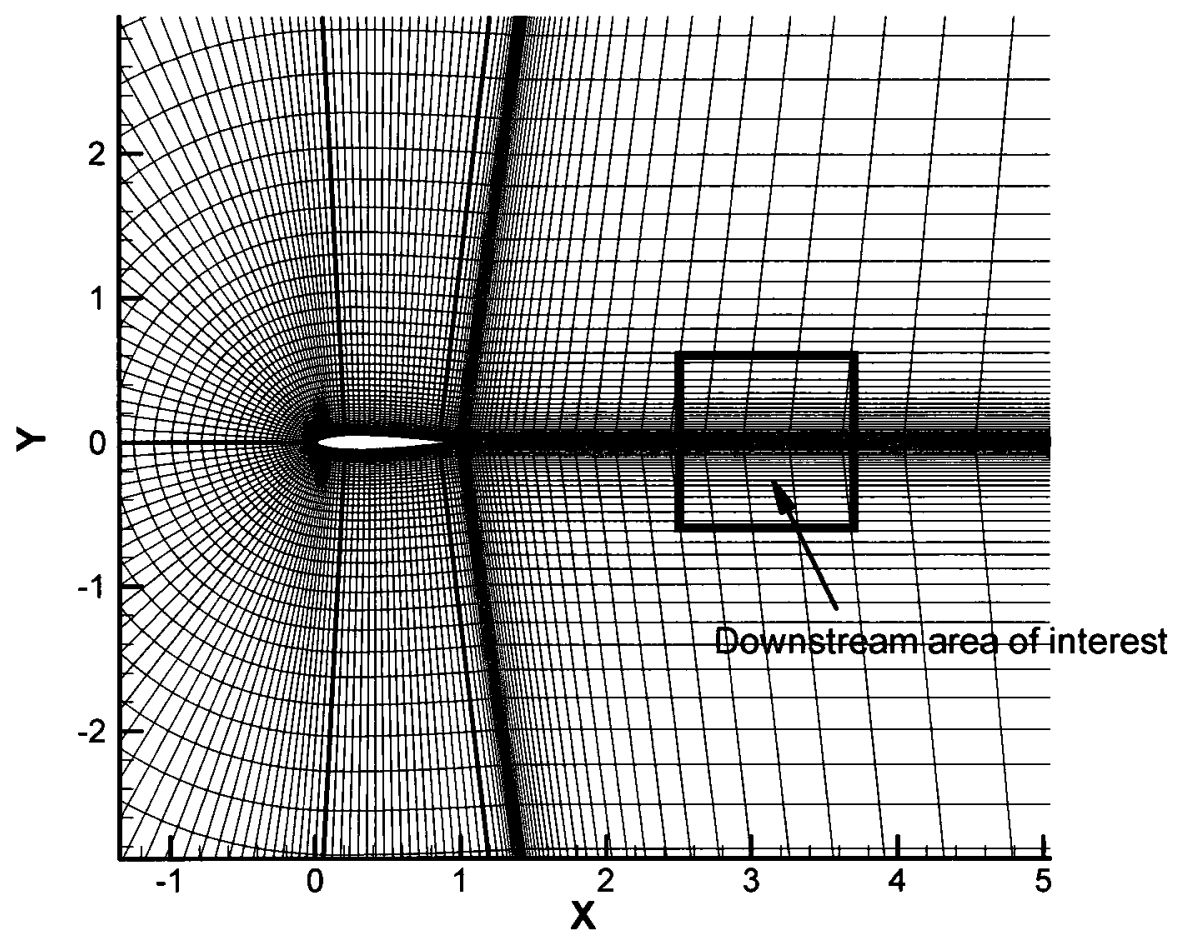

Figure 5.12: Downstream region of interest for vorticity preservation investigation with the Euler solver (CMB). 


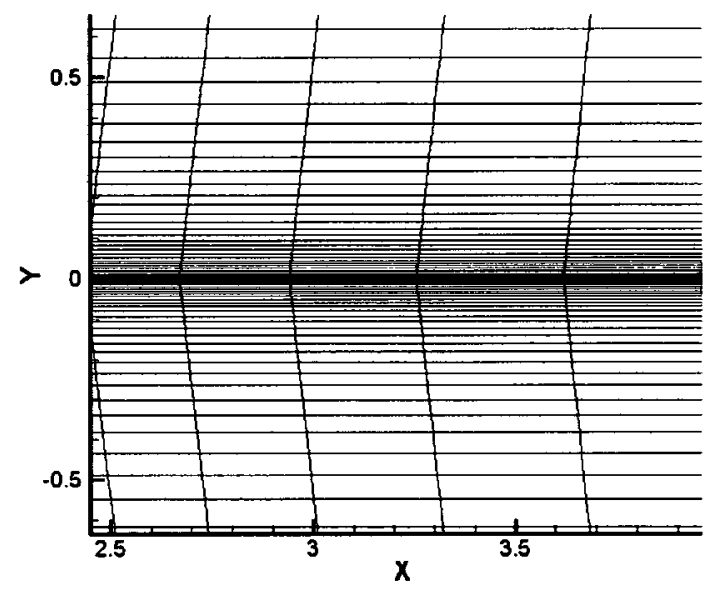

(a)

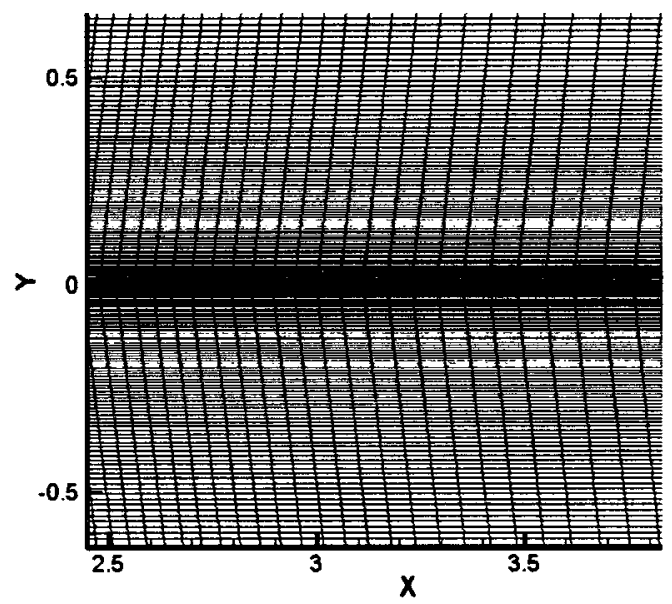

(c)

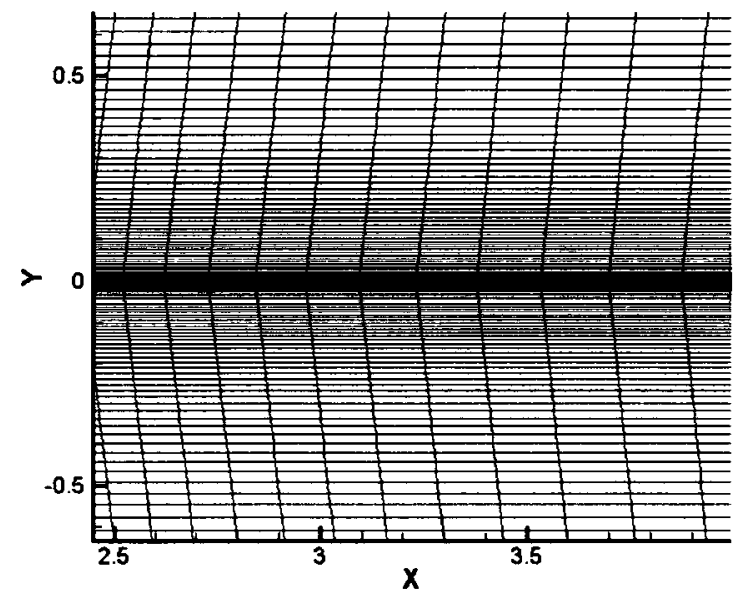

(b)

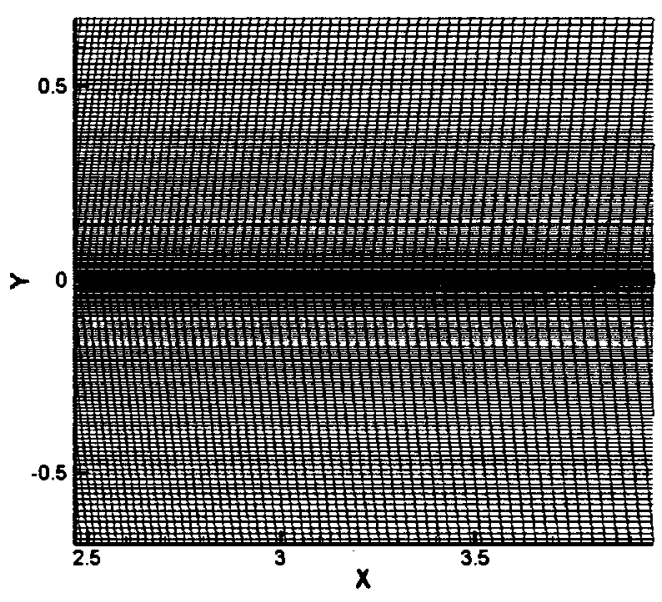

(d)

Figure 5.13: Euler grids for demonstrating numerical dissipation of vorticity. Grid densities are illustrated for the region of interest identified in Figure 5.12: (a) coarse (b) medium (c) fine (d) extra fine. 
Figure 5.14 displays the vorticity contours for the above grid densities. It is seen that the coarse grid does not capture the existence of a wake at this position, while only a trace at the start of the region of interest is noticed for the medium grid. In the fine grid it is clear that a vortex wake exist, although its structure is smeared and a large amount of numerical dissipation has occurred. As expected, the extra fine grid features the least dissipation with the von Kármán vortex street structure clearly shown. However, it is important to note that even across the area of interest, there is a large amount of dissipation taking place.

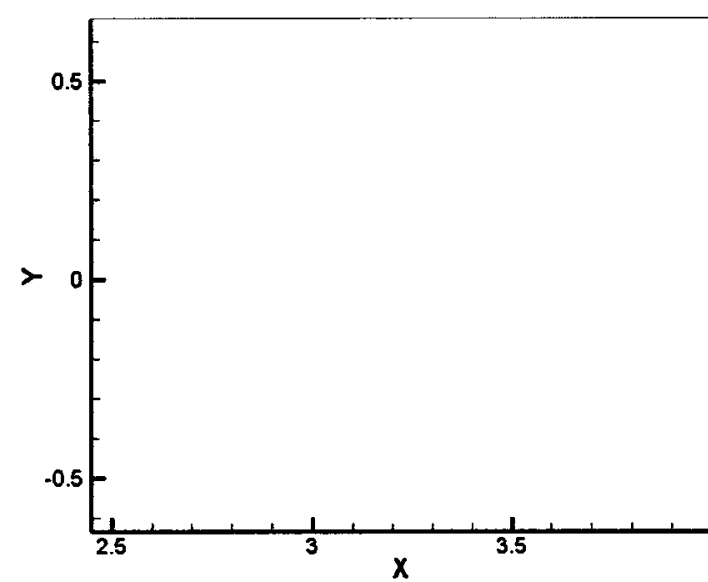

(a)

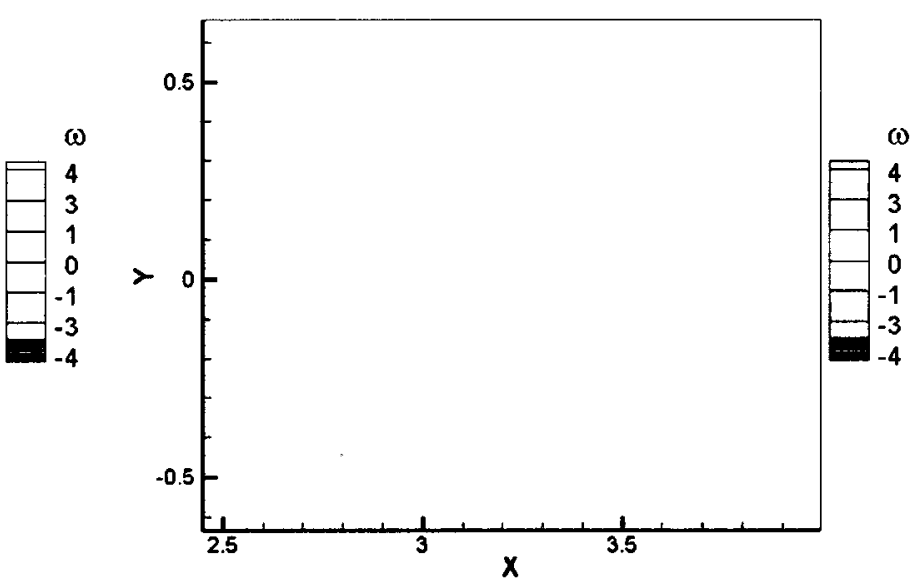

(b)

Figure 5.14: Vorticity contours on the Euler grids for (a) coarse (b) medium (c) fine and (d) extra fine densities. 


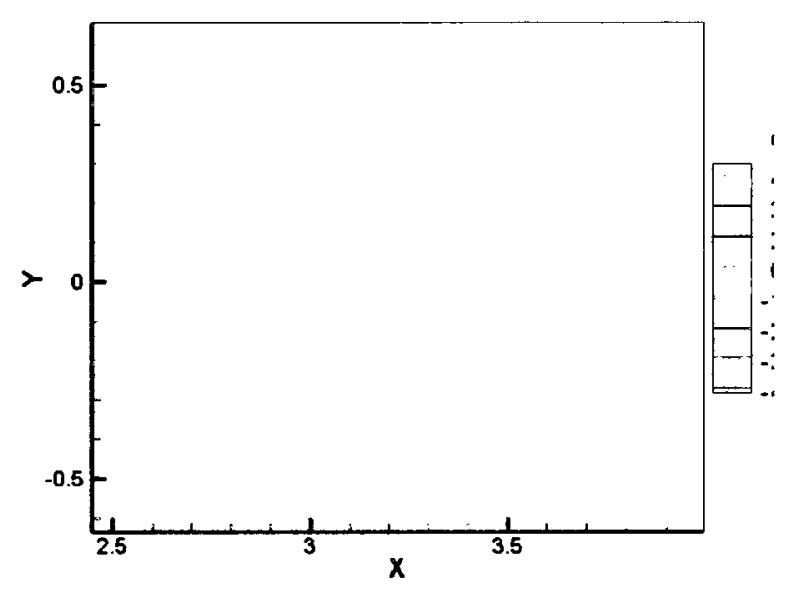

(c)

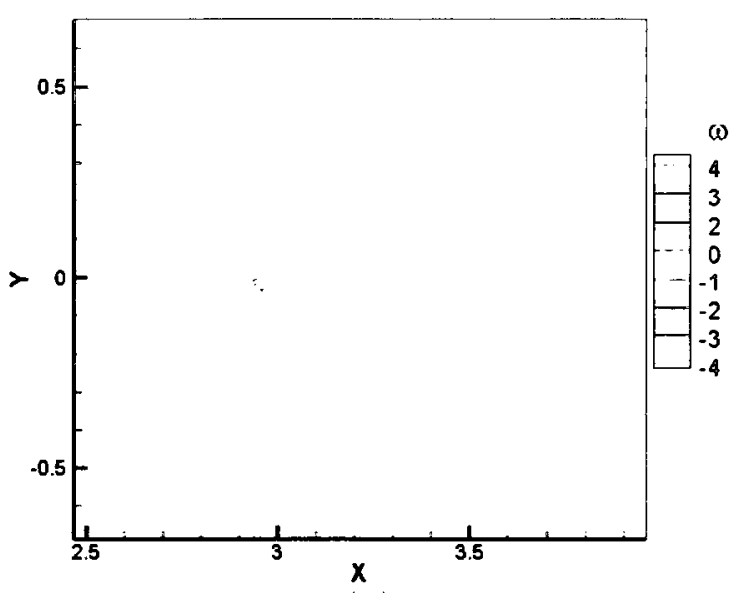

(d)

Figure 5.14: Vorticity contours on the Euler grids for (a) coarse (b) medium (c) fine and (d) extra fine densities. (cont)

The area of interest for HEPM, as shown in Figure 5.15, is in the same location as for the Euler investigation. For the UNTEADY1 case, this area is located within the CVPM domain, completely outside of the Euler domain. Figure 5.16 displays the vorticity contours in the area of interest for UNSTEADY1 case. The von Kármán vortex street structure is clearly shown. It is important to note that in contrast with even the extra fine Euler solution in Figure $5.14 \mathrm{~d}$, there is no numerical dissipation across the area of interest. This clearly illustrates the usefulness of HEPM for rotorcraft BVI applications. 


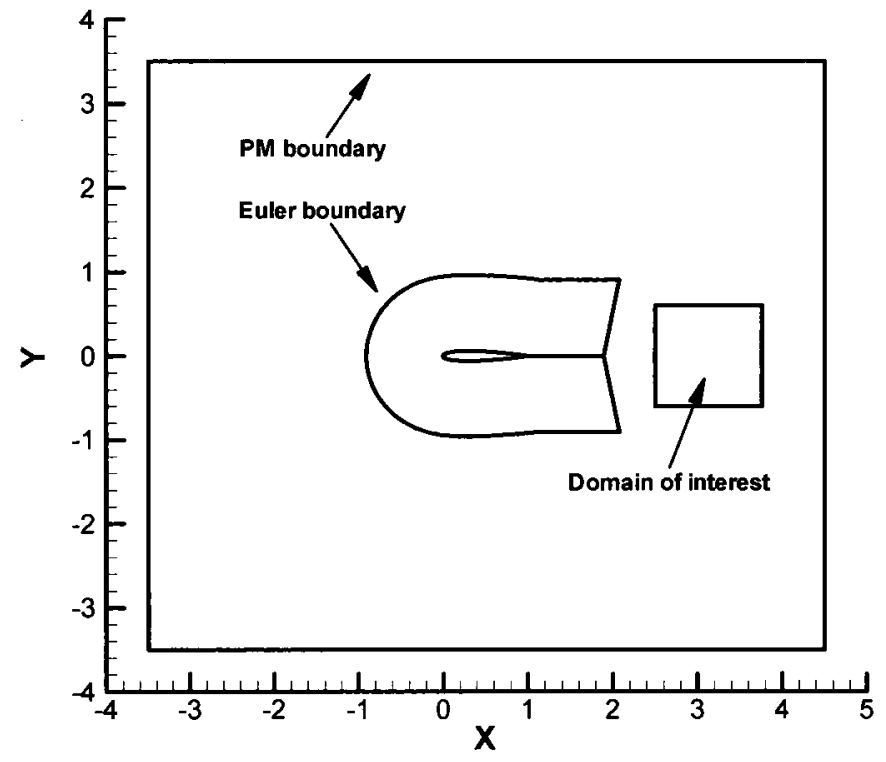

Figure 5.15: Downstream region of interest for vorticity preservation investigation with HEPM.

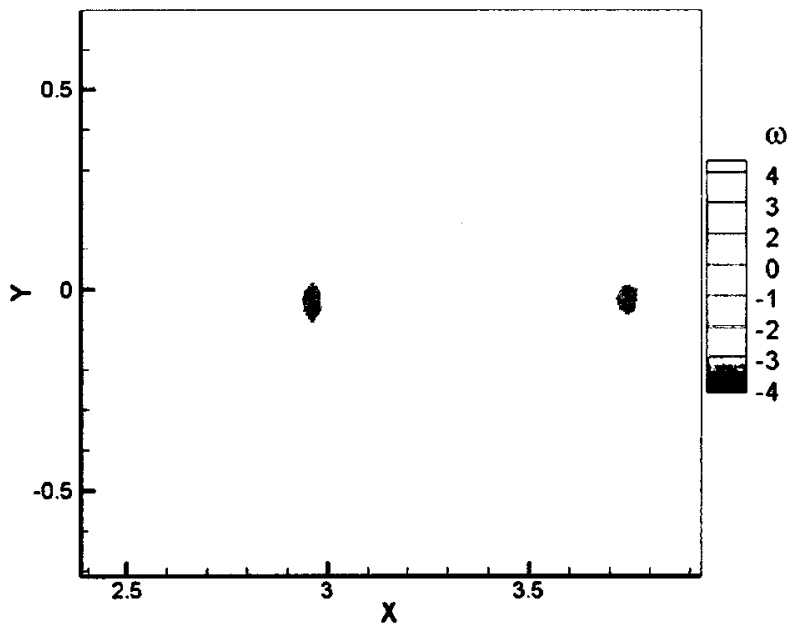

Figure 5.16: Vorticity contours in the above region of interest. 
Figure 5.17 further demonstrates this usefulness. Here, the vorticity on the extra fine Euler grid is compared with that of HEPM on the centreline of the domain of interest. It is clear that although the extra fine grid captures the vortex structure and strength nicely at first, the vortex has lost $25 \%$ of its strength and much of its structure as it leaves the domain. On the other hand, the HEPM UNSTEADY1 vortex strength and structure is preserved across the domain. It is noted that the magnitude of the HEPM vorticity at the beginning of the domain is of a smaller magnitude than that of the Euler grid. This is caused by the grid density employed for the reduced Euler grid in UNSTEADY1, which is of comparable density to that of the medium Euler grid. The discrepancy is due to numerical dissipation of this vortex in HEPM as it travels 1 chord length from the trailing edge before it leaves the Euler domain. 


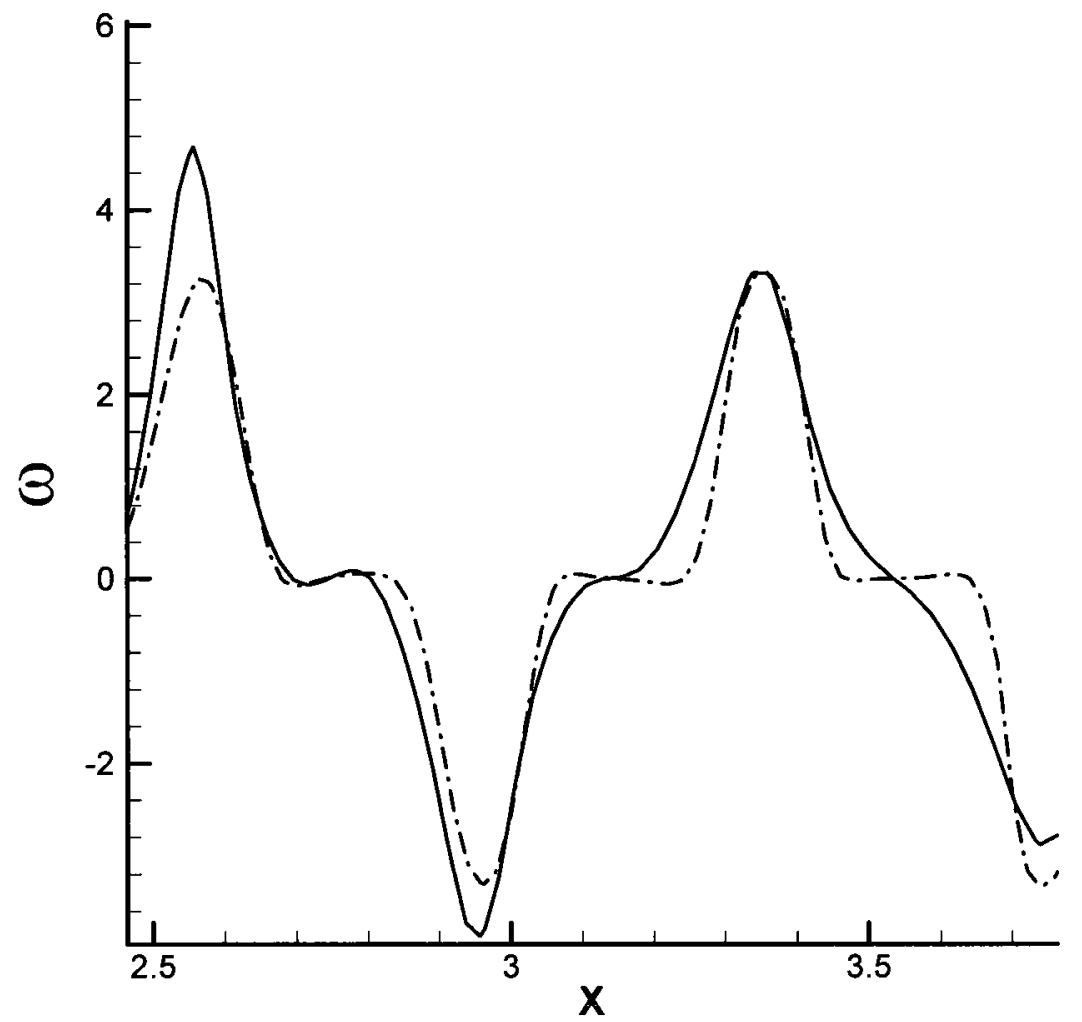

Figure 5.17: Vorticity distribution at $\mathrm{y}=0$ across the domain of interest for the extra fine Euler grid (solid line) and for HEPM (dashed line). 


\section{$5.2 \quad$ Validation}

Validation of the HEPM code in 2D was done using the Compendium for Unsteady Aerodynamic Measurements published by the Advisory Group for Aerospace Research and Development (AGARD) in AGARD Report 707 [56]. The main purpose of these tests was to examine the conditions of dynamic stall and recovery at scaled time rates similar to those of typical helicopter applications. Although HEPM is an inviscid code incapable of predicting dynamic stall, the scaled time rates of these AGARD tests make them ideal comparison data considering the transonic rotorcraft applications intended for HEPM. Table 5.6 lists the experimental specifications for these experiments. Selected for comparisons are the AGARD CT6 and CT5 cases.

Table 5.6: AGARD experimental specifications

\begin{tabular}{l|l}
\hline \hline Airfoil & N0012 \\
Chord & $101.6 \mathrm{~mm}$ \\
Span & $203.2 \mathrm{~mm}$ \\
Motion & ramped-up and sinusoidal pitch motion \\
Tunnel & ARA 2-D tunnel \\
Type & Intermittent blow down \\
Dimensions & height $=457.2$, width $=203.2$, length $=1251 \mathrm{~mm}$ \\
\hline \hline
\end{tabular}

\subsubsection{AGARD CT6 - Ramping Pitch Motion}

AGARD CT6 is a ramped-up pitch motion about $0.25 \mathrm{c}$. This HEPM case is designated as UNSTEADY2 and the input parameters are listed in Table 5.7.

Table 5.8 gives the properties for this motion. 
Table 5.7: HEPM input parameters: UNSTEADY2

\begin{tabular}{ll|ll|ll}
\hline \hline General & & Euler solver & & Particle method & \\
\hline Airfoil & N0012 & Grid density & 10000 cells & $X_{\min , \max }$ & $-3.5,4.5$ \\
Chord & $0.1016 \mathrm{~mm}$ & Extent & 1 chord & $Y_{\min , \max }$ & $-2.5,2.5$ \\
Mach & 0.30 & Implicit CFL & 150 & PM $\Delta h$ & 0.02 \\
Motion & see Table 5.8 & Steady $\varepsilon_{E}$ & $1 \mathrm{E}-6$ & Particles/cell & 4 \\
$\Delta t$ & 0.007 & Unsteady $\varepsilon_{E}$ & $1 \mathrm{E}-3$ & Project/Interp & TSC \\
\hline \hline
\end{tabular}

Table 5.8: AGARD CT6 validation case

\begin{tabular}{c|c|c|c|c}
\hline \hline $\begin{array}{c}\text { Related AGARD } \\
\text { CT Case }\end{array}$ & $\mathrm{M}$ & $\begin{array}{c}\alpha \text { range } \\
(\mathrm{deg})\end{array}$ & $\begin{array}{c}d \alpha / d t \\
(\mathrm{deg} / \mathrm{s})\end{array}$ & $\begin{array}{c}\mathrm{Re} \\
\left(10^{-6}\right)\end{array}$ \\
\hline 6 & 0.30 & $-0.03-15.54$ & 1280 & 2.7 \\
\hline \hline
\end{tabular}

Figure 5.18 presents $C_{n}$ as a function of $\alpha$. It is seen that the trend is correctly predicted by HEPM with small variations occurring as $\alpha$ increases. The reason for this is demonstrated in Figure 5.19, which presents the surface $C_{p}$ distribution variations over time. It is clear that the suction peak is smaller for both numerical results, suggesting that the discrepancy originates from the Euler solver. HEPM is unable on its own to improve the results that the Euler solver is delivering, so this comparison is important. It is noted that the Euler solution undergoes oscillations on startup, which is not present in the HEPM solution. This is due to a difference in initialization, where the Euler solver is initialized with freestream values, while HEPM starts from a steady solution. Finally, it is seen that the Euler solver becomes less effective than HEPM as $\alpha$ increases due to the numerical dissipation of the wake with time. 


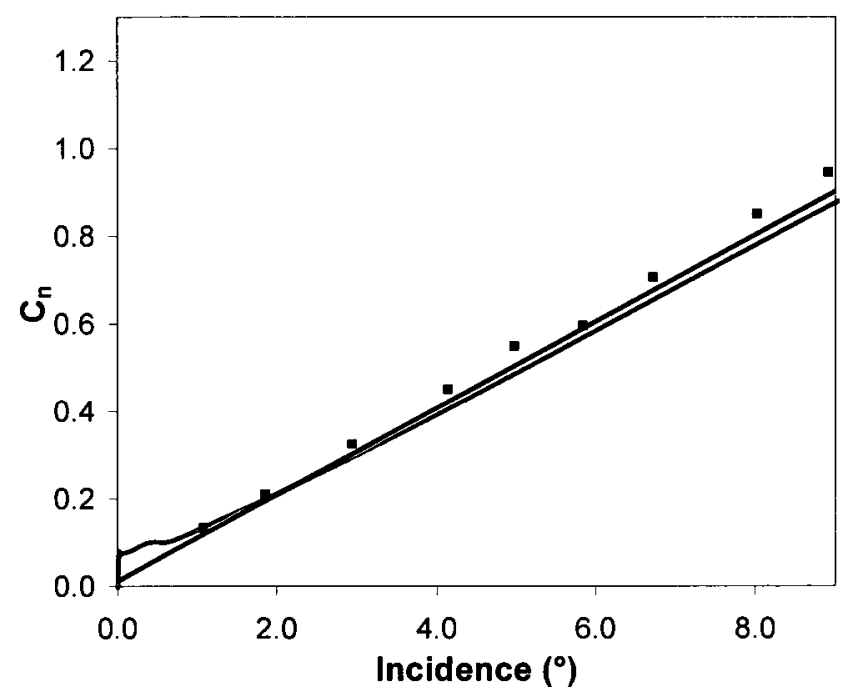

Figure 5.18: Comparison of normal force history between experimental AGARD CT6 (square), HEPM UNSTEADY2 (black line) and Euler solution (red line).
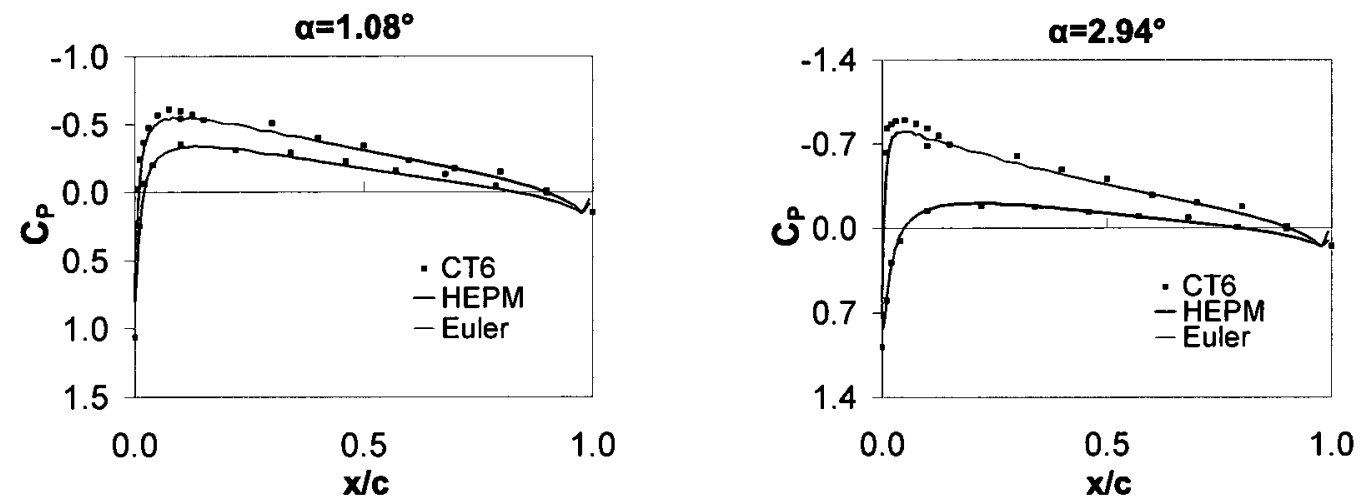

Figure 5.19: Pressure coefficient distributions for experimental AGARD CT6, HEPM UNSTEADY2 and Euler solution. 

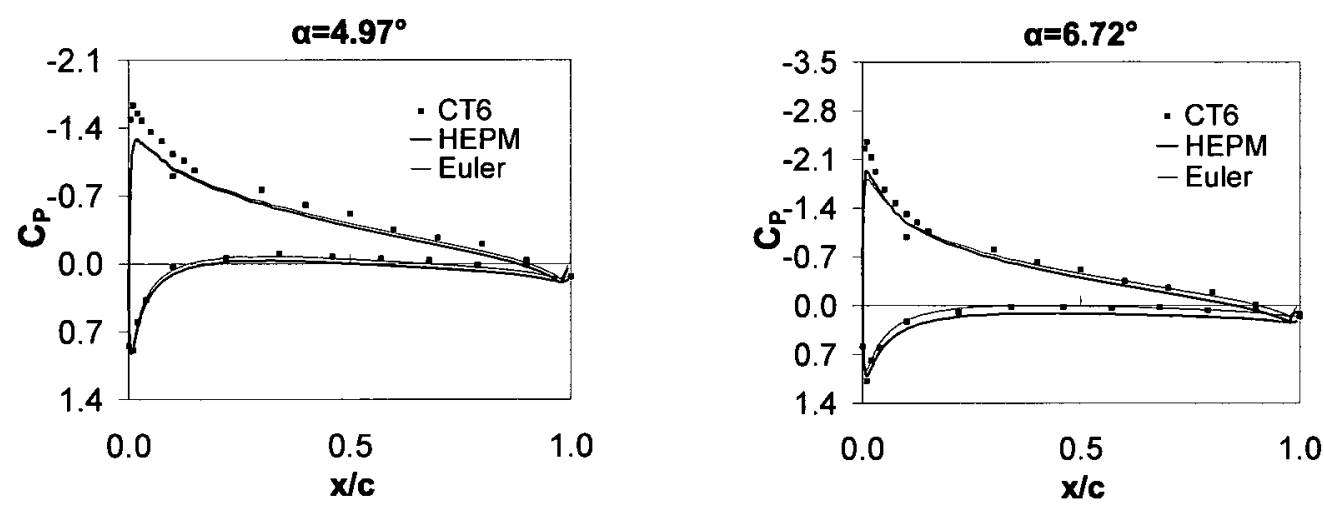

Figure 5.19: Pressure coefficient distributions for experimental AGARD CT6, HEPM UNSTEADY2 and Euler solution. (cont)

\subsubsection{AGARD CT5 - Sinusoidal Pitch Motion}

AGARD CT5 is a sinusoidal pitch case about $0.25 \mathrm{c}$ according to the equation $\alpha=$ $\alpha_{m}+\alpha_{0} \sin 2 k t$. This HEPM case is designated as UNSTEADY3 with input parameters listed in Table 5.9.

Table 5.9: HEPM input parameters: UNSTEADY3

\begin{tabular}{ll|ll|ll}
\hline \hline General & & Euler solver & & Particle method & \\
\hline Airfoil & N0012 & Grid density & 10000 cells & $X_{\min , \max }$ & $-3.5,4.5$ \\
Chord & $0.1016 \mathrm{~mm}$ & Extent & 1 chord & $Y_{\min , \max }$ & $-2.5,2.5$ \\
Mach & 0.755 & Implicit CFL & 60 & PM $\Delta h$ & 0.02 \\
Motion & see Table 5.10 & Steady $\varepsilon_{E}$ & $1 \mathrm{E}-6$ & Particles/cell & 4 \\
$\Delta t$ & 0.007 & Unsteady $\varepsilon_{E}$ & $1 \mathrm{E}-3$ & Project/Interp & TSC \\
\hline \hline
\end{tabular}

Table 5.10 displays the motion parameters implemented where $k$ is reduced frequency. 
Table 5.10: AGARD CT5 validation case

\begin{tabular}{c|c|c|c|c|c|c}
\hline $\begin{array}{c}\text { Related AGARD } \\
\text { CT Case }\end{array}$ & $\mathrm{M}$ & $\begin{array}{c}\alpha_{m} \\
(\text { deg. })\end{array}$ & $\begin{array}{c}\alpha_{0} \\
(\text { deg. })\end{array}$ & $\begin{array}{c}\mathrm{f} \\
(\mathrm{Hz})\end{array}$ & $\mathrm{k}$ & $\begin{array}{c}\mathrm{Re} \\
\left(10^{-5}\right)\end{array}$ \\
\hline 5 & 0.755 & 0.016 & 2.51 & 62.5 & 0.0814 & 5.5 \\
\hline \hline
\end{tabular}

Figure 5.20 presents the convergence history for the lift hysteresis loop. As expected, it is found that results converge after about 3 cycles. In Figure 5.21 the converged results are presented for the third $C_{n}$ hysteresis loop. It is found that the HEPM results agree well with the Euler reference solution, although the experimental results are slightly underpredicted. The pitch motion has a negligible mean value, indicating the hysteresis loop centre should be at the axis origin for the 2D case.

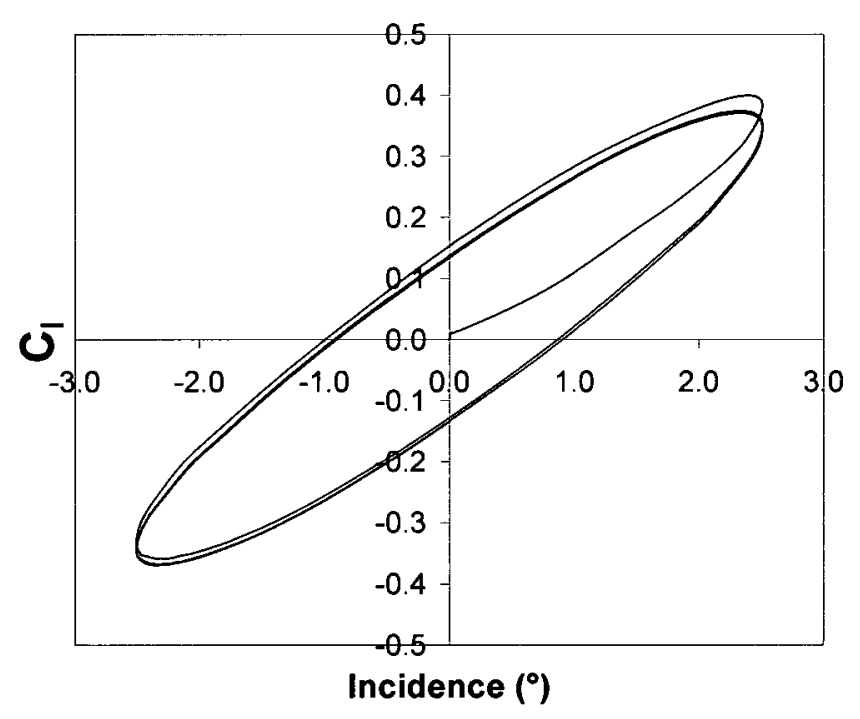

Figure 5.20: Lift hysteresis convergence history for HEPM UNSTEADY3. 


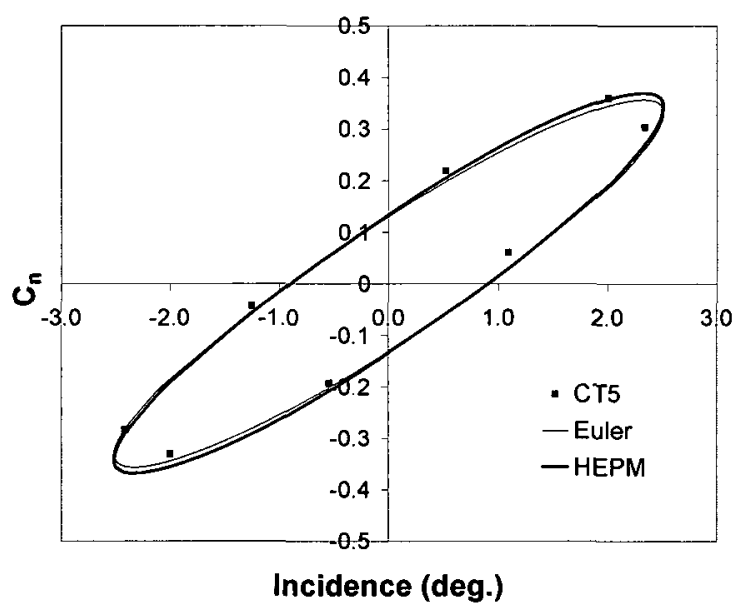

Figure 5.21: Comparison of converged normal force over the 3rd cycle for the experimental AGARD CT5 (square), Euler reference (red line) and HEPM UNSTEADY3 (black line) solution.

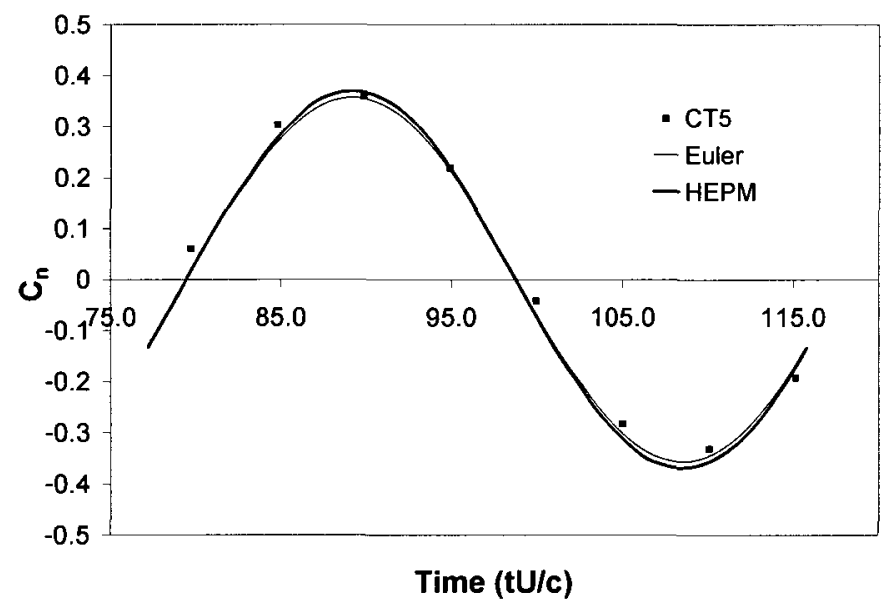

Figure 5.22: Comparison of the converged normal force time history over the 3rd cycle for experimental AGARD CT5 (square), Euler reference (red line) and HEPM UNSTEADY3 (black line) solutions. 
Figure 5.23 presents the surface $C_{p}$ distributions at several time points during the converge cycle. It is found that HEPM correctly predicts these pressures, with good shock capturing at all points during the motion. This is very important since it demonstrates the suitability of HEPM for compressible rotorcraft applications.
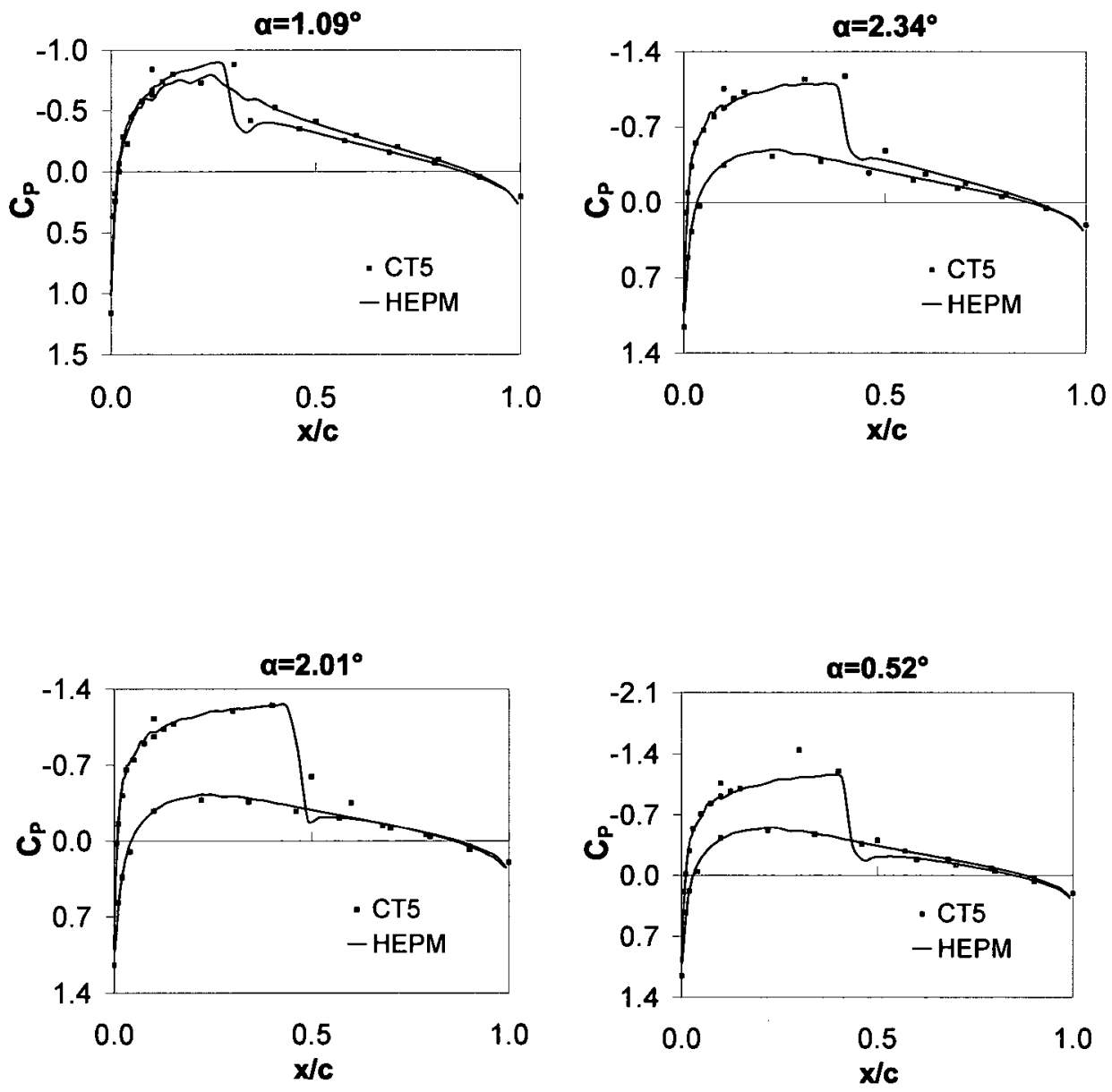

Figure 5.23: Pressure coefficient distributions for AGARD CT5 case. 

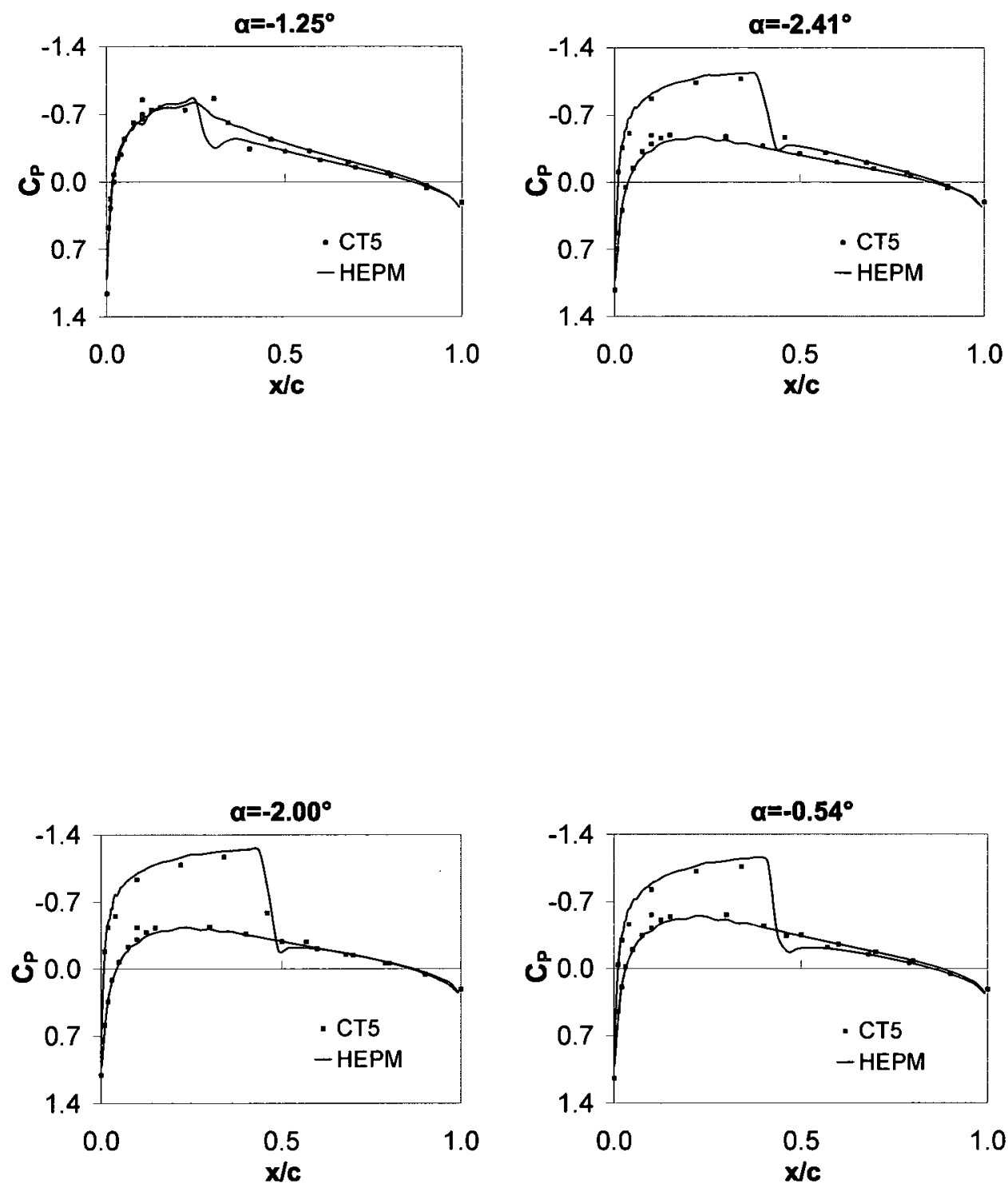

Figure 5.23: Pressure coefficient distributions for AGARD CT5 case. (cont) 


\subsection{Airfoil-Vortex Interaction}

An important aspect of rotorcraft aerodynamics is blade-vortex interaction (BVI), whose simplified representation in 2-D is airfoil-vortex interaction (AVI). AVI results in the literature are available for conventional CFD by Wong and Nitzsche [57], for vorticity-preserving schemes by Falissard and Lerat [58] and vorticity-confinement schemes by Morvant et al. [59]. These simulations suffered from the following major problems:

1. vortex wandering on a conventional CFD code, in which the impinging vortex undergoes vertical oscillations as it approaches the airfoil [57];

2. vortex dissipation, where the vortex strength numerically dissipates significantly by the time it reaches the airfoil [57-59];

3. need for the fine-tuning of a case-dependent vorticity confinement parameters in vorticity-confinement schemes [59];

4. extremely large computational costs [57-59];

5. unrealistic vortex size and strength for rotorcraft applications [57-59].

For point 5, note that the numerical comparisons are based on the experimental work by Lee and Bershader [60], with the impinging vortex being formed by the diffraction of a shock across a NACA0018 airfoil. The vortex in these 2-D experiments 
is modeled as a Kaufmann/Scully vortex with a swirl velocity, $V_{\theta}$ given as

$$
V_{\theta}=\frac{\Gamma_{v}}{2 \pi r}\left(\frac{r^{2}}{r^{2}+r_{c}^{2}}\right)
$$

where $r$ is the radial distance from the centre of the vortex. The strength, $\Gamma_{v}$, and core size, $r_{c}$, were determined experimentally to be $\Gamma_{v}=-0.283$ and $r_{c}=0.018$ respectively [60]. However, it is clear that this is not realistic for rotorcraft applications. The vortex cited above is far too strong and the core size is too small for realistic rotorcraft simulations. Typically, it is found that a rotorcraft tip vortex core size is 0.05-0.15 chord lengths, while Leishman [7] gives a relation for the expected vortex strength as:

$$
\Gamma_{v}=A \Omega R c\left(\frac{C_{T}}{\sigma}\right)
$$

where $A=2, \Omega, R$ and $c$ are the rotor speed, span and chord respectively. $C_{T}$ is the thrust coefficient and $\sigma$ is the solidity ratio given by

$$
\sigma=\frac{N_{b} c}{\pi R}
$$

where $N_{b}$ is the number of blades. In order to create an impinging vortex representative of typical rotorcraft applications, the parameters available for the HELINOISE experiments [61], conducted at the DNW wind tunnel, are used. This experimental data is widely accepted in the rotorcraft community as benchmark results. The HELINOISE parameters used are presented in Table 5.11 


\begin{tabular}{l|l} 
Table 5.11: HELINOISE 947 \\
\hline \hline Flight condition & hover \\
$N_{b}$ & 4 \\
$R$ & $2 \mathrm{~m}$ \\
$c$ & $0.121 \mathrm{~m}$ \\
$C_{T}$ & 0.00436 \\
$\Omega$ & $110 \mathrm{rad} / \mathrm{s}$ \\
\hline
\end{tabular}

By using these parameters, we arrive at an impinging vortex strength and core size of $\Gamma_{v}=0.1456$ and $r_{c}=0.10$.

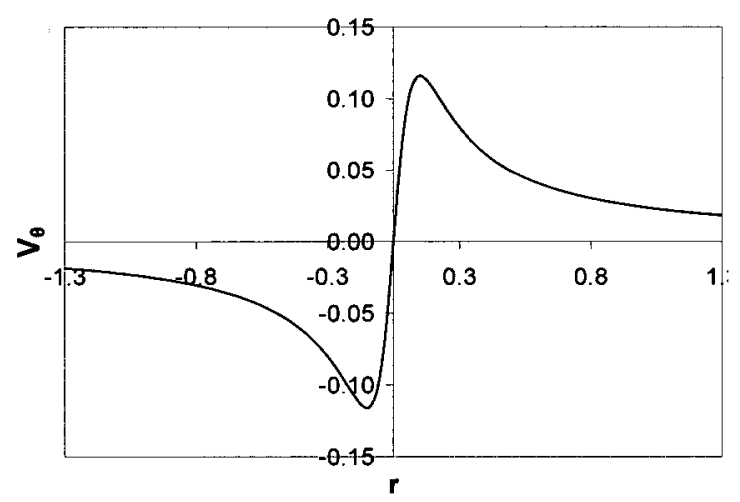

(a)

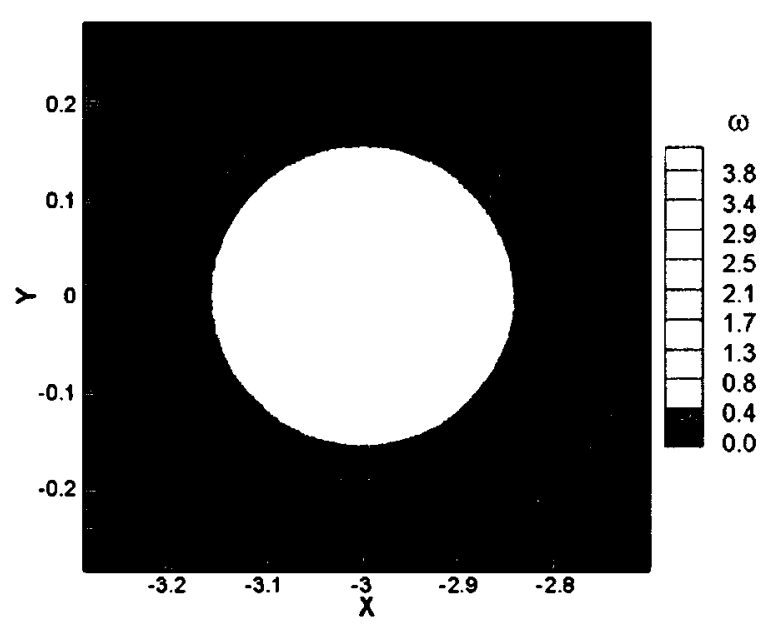

(b)

Figure 5.24: Core distribution of swirl velocity (a) and associated vorticity contours (b) for the vortex initialized in the AVI studies.

Figure 5.25 illustrates the HEPM computational domain used in the AVI studies. The vortex is initialized 3 chord lengths in front of the airfoil leading edge at a specified vertical miss distance, $e$. This configuration was then studied at both low $(\mathrm{Ma}=0.5)$ and higher $(\mathrm{Ma}=0.8)$ Mach numbers, as will be detailed in the next sections. Note 
that with the lack of experimental results, comparison with measurements will not be possible for these test cases. However, general trends observed during the tests should give us sufficient confidence in the simulations. The aim is not to provide detailed insight into the mechanism of AVI, but to demonstrate that HEPM can tackle such complex flow phenomena.

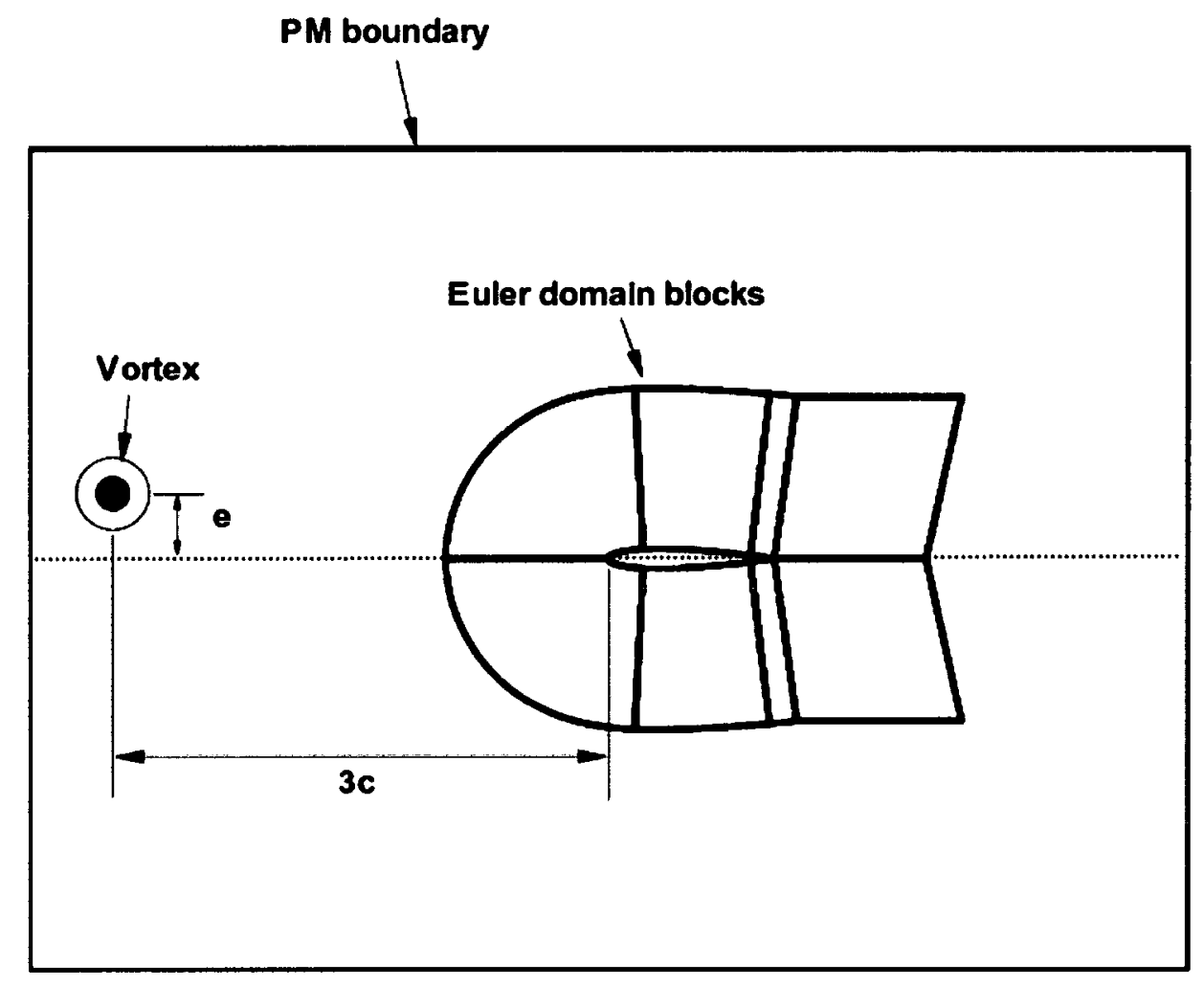

Figure 5.25: HEPM model for AVI studies. 


\subsubsection{Low Mach Number AVI Simulation (AVI1)}

Due to the inviscid nature of the analysis, the important interactions between the impinging vortex and the boundary layer will not be considered. For this reason, only the global variables, such as $C_{l}$ and $C_{p}$ at particular stations will be examined instead of the detailed mechanics of AVI. The input parameters for the first case, AVI1, can be found in Table 5.12. For all AVI simulations, the airfoil is held at $\alpha=0^{\circ}$. Note the fine grid used for the Euler domain. Although HEPM implements a reduced grid extending only 1 chord length from the airfoil surface, the impinging vortex will still have to travel approximately a distance of 3 chord lengths in order to pass through the Euler domain. As such, it is necessary to implement an extremely fine grid for AVI studies in order to minimize the numerical dissipation in the Euler region.

Table 5.12: HEPM input parameters: AVI1

\begin{tabular}{ll|ll|ll}
\hline \hline General & & Euler solver & & Particle method & \\
\hline Airfoil & N0012 & Grid density & 95500 cells & $X_{\min , \max }$ & $-4.5,4.5$ \\
Chord & $0.1016 \mathrm{~mm}$ & Extent & 1 chord & $Y_{\min , \max }$ & $-3.5,3.5$ \\
Mach & 0.50 & Implicit CFL & 105 & PM $\Delta h$ & 0.02 \\
Motion & steady & Steady $\varepsilon_{E}$ & $1 \mathrm{E}-6$ & Particles/cell & 4 \\
$\Delta t$ & 0.007 & Unsteady $\varepsilon_{E}$ & $1 \mathrm{E}-3$ & Project/Interp & TSC \\
\hline \hline
\end{tabular}

In Figure 5.26 the vorticity contours can be tracked as the AVI interaction progresses. Simulations were run for 5.2 non-dimensional time units allowing the vortex to convect 3 chord lengths to reach the airfoil, pass over it and subsequently convect 
for 1.2 chord lengths beyond it. Figure 5.27 presents the lift coefficient history during the interaction. The mechanism of interaction begins as the vortex approaches the airfoil. At this point, for the positive vortex strength, an upwash velocity is induced at the leading edge, causing the stagnation point to move downwards along the lower surface creating an increase of lift. As the vortex moves along the length of the airfoil, the induced velocity is reversed and the leading edge experiences downwash, moving the stagnation point back towards the upper surface of the blade, reducing the lift. The vortex then reaches the end of the airfoil and begins to interact with the wake generating the perturbations. Experience showed that the flow returns to a steady state for longer runtimes. It is observed that as the miss distance is increased, the amplitude effect of the interaction decreases. Figures 5.28 and 5.29 present the $C_{p}$ histories at a point just behind the leading edge at chordwise position $x / c=0.055$ for the upper and lower surfaces. 


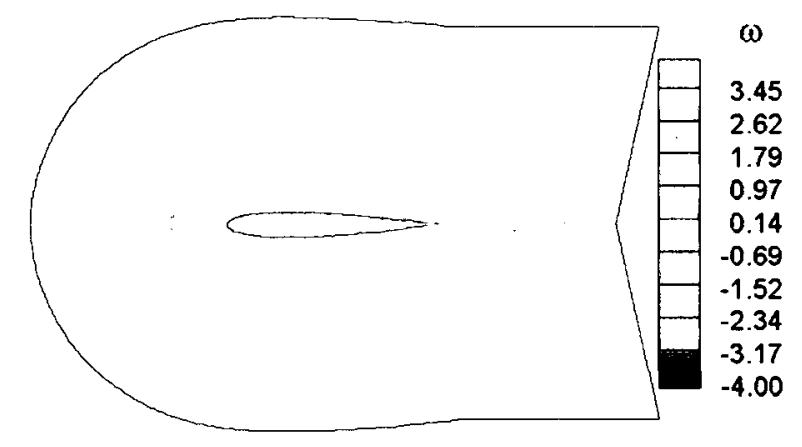

$t=2.7$

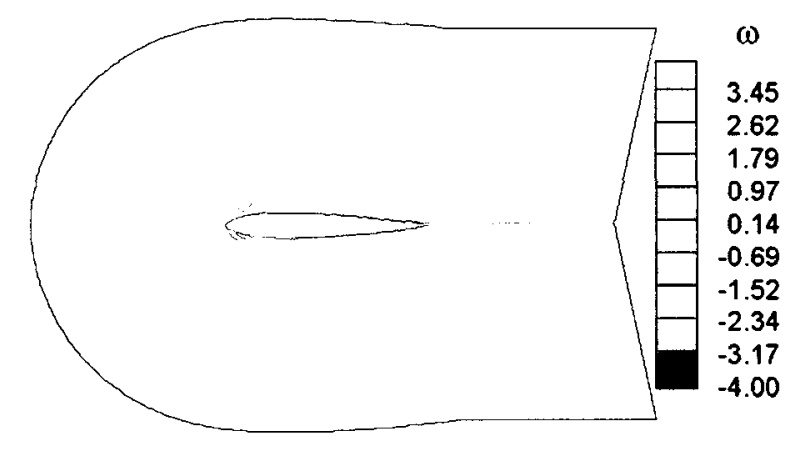

$t=3.2$

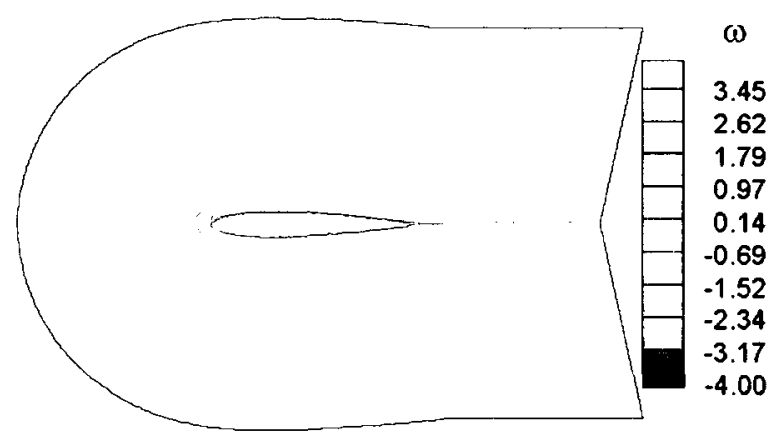

$t=3.0$

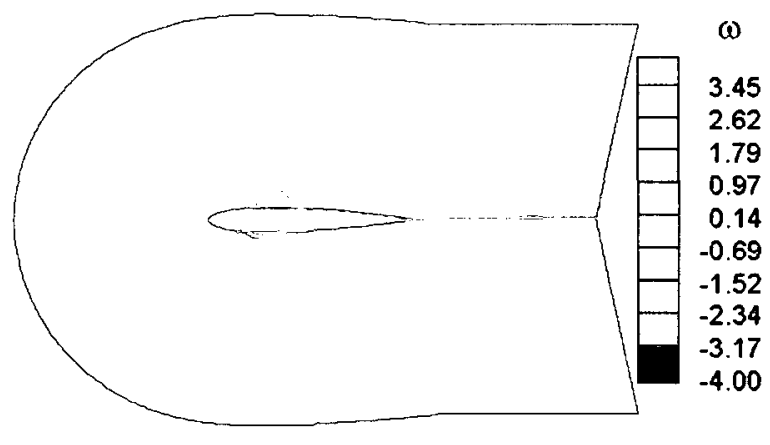

$t=3.4$

Figure 5.26: Vorticity contours for the AVI1 case $(\mathrm{e}=0.0)$. 


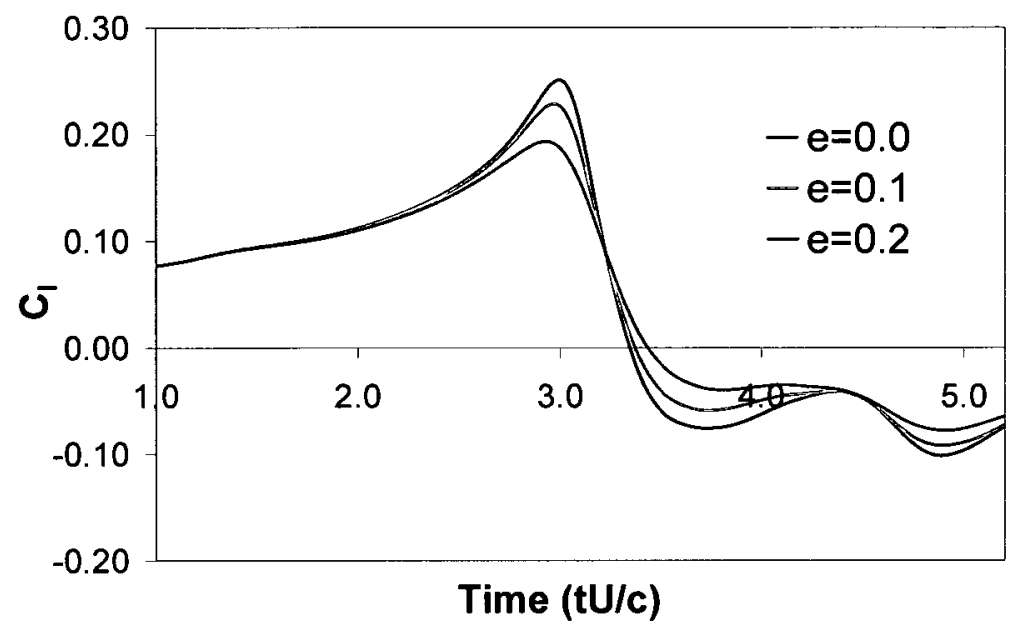

Figure 5.27: Lift coefficient history for AVI1 case at various vortex miss distances.

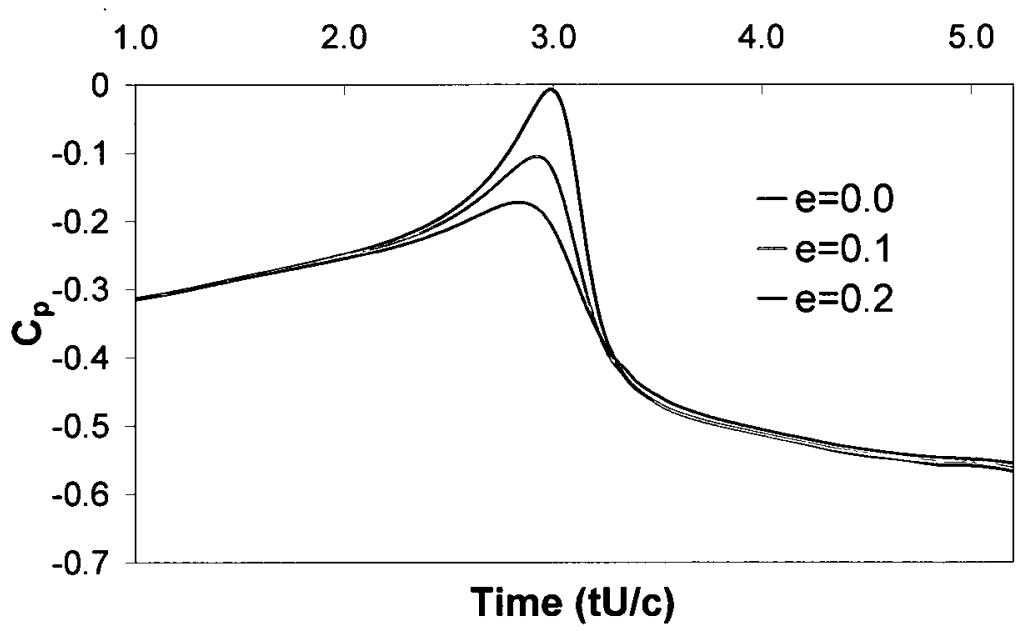

Figure 5.28: Pressure coefficient history at upper $\mathrm{x} / \mathrm{c}=0.055$ position for AVI1 case at various vortex miss distances. 


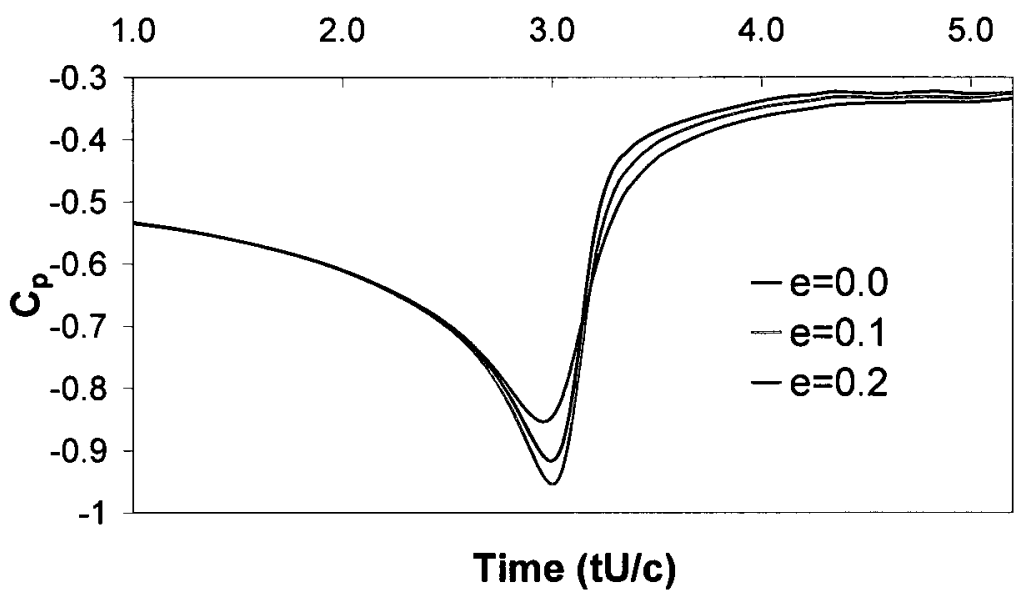

Figure 5.29: Pressure coefficient history at lower $\mathrm{x} / \mathrm{c}=0.055$ position for AVI1 case at various vortex miss distances. 


\subsubsection{High Mach Number AVI Simulation (AVI2)}

Table 5.13 lists the HEPM parameters used in the AVI2 case. This is a transonic case featuring shock waves on the upper and lower surface of the airfoil. Figures 5.30, 5.31 and 5.32 present the same $C_{l}$ and $C_{p}$ histories for the transonic case at Ma=0.8. The mechanisms of the interaction remain the same as for AVI1. As the impinging vortex approaches the airfoil an upwash velocity is induced at the leading edge, causing the stagnation point to move downwards along the lower surface creating an increase of lift. As the vortex moves along the length of the airfoil, the induced velocity is reversed and the leading edge experiences downwash, moving the stagnation point back towards the upper surface of the blade, reducing the lift. The vortex then reaches the end of the airfoil and begins to interact with the wake generating the perturbations. These perturbations are of a larger magnitude than with the low Mach number case. Again, it is observed that as the miss distance is increased, the amplitude effect of the interaction decreases.

Table 5.13: HEPM input parameters: AVI2

\begin{tabular}{ll|ll|ll}
\hline \hline General & & Euler solver & Particle method & \\
\hline Airfoil & N0012 & Grid density & 95500 cells & $X_{\min , \max }$ & $-4.5,4.5$ \\
Chord & $0.1016 \mathrm{~mm}$ & Extent & 1 chord & $Y_{\min , \max }$ & $-3.5,3.5$ \\
Mach & 0.80 & Implicit CFL & 25 & PM $\Delta h$ & 0.02 \\
Motion & steady & Steady $\varepsilon_{E}$ & $1 \mathrm{E}-6$ & Particles/cell & 4 \\
$\Delta t$ & 0.007 & Unsteady $\varepsilon_{E}$ & $1 \mathrm{E}-3$ & Project/Interp & TSC \\
\hline \hline
\end{tabular}




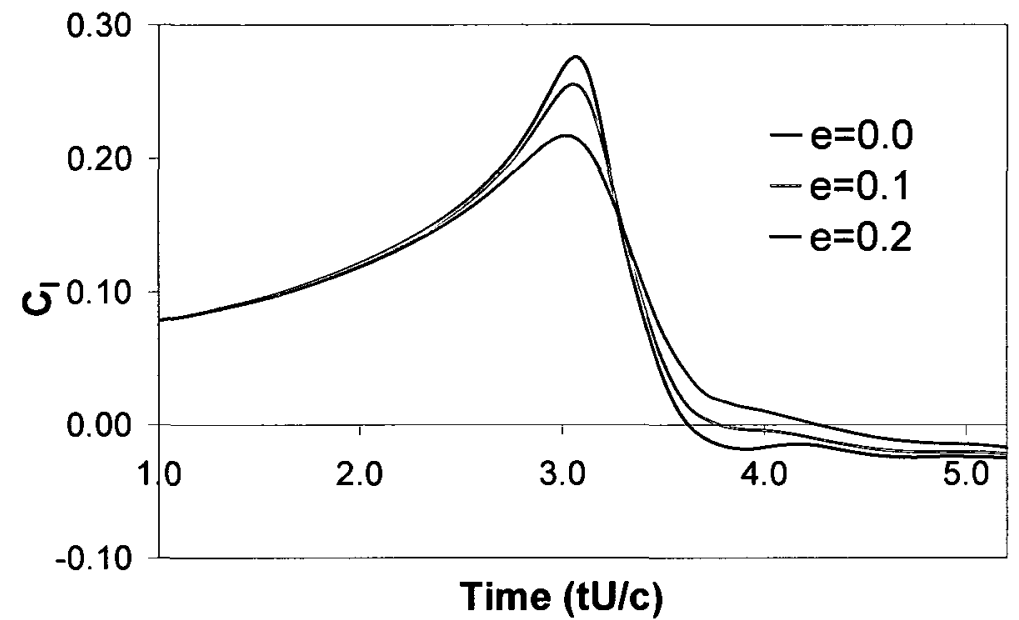

Figure 5.30: Lift coefficient history for AVI2 case at various vortex miss distances.

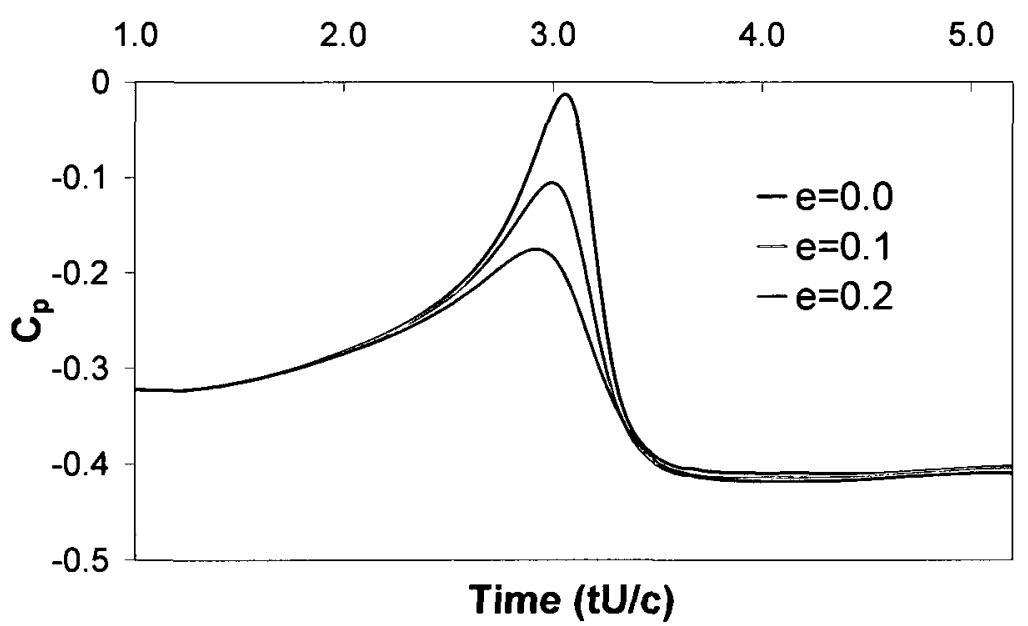

Figure 5.31: Pressure coefficient history at upper $\mathrm{x} / \mathrm{c}=0.055$ position for AVI2 case at various vortex miss distances. 


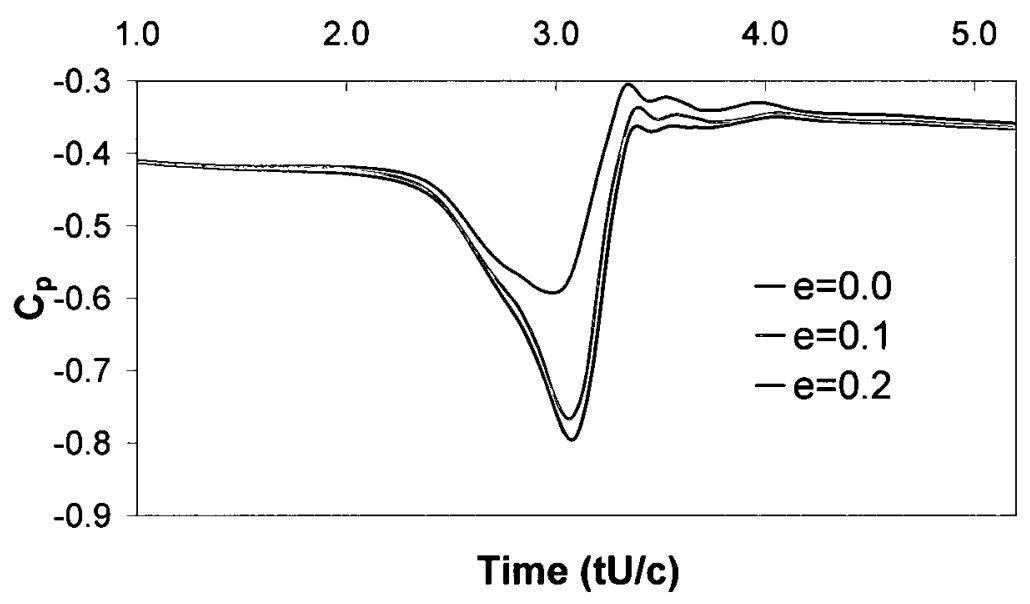

Figure 5.32: Pressure coefficient history at lower $\mathrm{x} / \mathrm{c}=0.055$ position for AVI2 case at various vortex miss distances. 


\section{Chapter 6}

\section{Conclusions and Recommendations}

\subsection{Conclusions}

The primary aim of the present work was to lay down the $2 \mathrm{D}$ foundations of a novel CFD method dedicated to rotorcraft applications and featuring the following combined capabilities for the first time:

- removal of the need for a complex meshing strategy by eliminating the requirement for connectivity between rotating and non-rotating rotorcraft components;

- exact treatment of the airfoil surface boundary conditions;

- high resolution of flow details in the near-blade region;

- numerical dissipation-free vorticity convection in the far-field.

Three main issues were addressed: the development of a compressible formulation for the vortex particle method, a coupling strategy for blending this compressible 
vortex particle method with an existing finite-volume Euler solver and verification and validation of the new hybrid CFD method.

The compressible formulation for the vortex particle method, designated as CVPM, was developed in Chapter 2. A compressible model was clearly necessary for this type of hybrid method. The Euler solver would be of sufficient extent to contain all expected shocks (approximately 1 chord length from the airfoil), but would certainly not extend into an incompressible flow regime. As such, the boundary conditions for the Euler solver, obtained from the vortex method, were required to be compressible in nature. Failure to properly account for mass continuity through the density boundary conditions in the Euler solver would result in a mass build-up inside the Euler domain, followed by the over-prediction of results, and eventual code failure.

As identified in the literature review, there had been little research on compressible models for particle methods, with only three existing research efforts. The particlemesh formulation of Meske, and the pure particle approach by Eldredge had limited applicability identified explicitly by their authors. This left an approach proposed by Mas-Gallic over 15 years ago, which had been validated for irrotational compressible flow only. As part of this work, this approach has been used as the basis for formulating a hybrid method applicable to 2D external vortical flows involving solid boundaries. With this new formulation, particles carrying mass and circulation could convect and dilate along material elements in a Lagrangian fashion, while 
compressibility effects needed to be solved for on an overlying Cartesian mesh in the Eulerian frame. However, this was not seen as a disadvantage, as an overlying mesh was required anyways for the fast Particle-Mesh technique required to improve the computational efficiency of the velocity field calculation.

In Chapter 4, the novel CVPM is dynamically coupled with an existing Euler solver (CMB) to create the unique hybrid Euler-compressible vortex particle method, designated as HEPM. This coupling employs the domain decomposition technique featuring complete overlap of the two numerical methods. At each time step there is an iteration process in order to ensure compatibility between the Euler and particle method solutions. Problems were encountered when outgoing dilatation waves reflected back into the computational, thereby degrading the solution. As such, nonreflective boundary conditions were successfully implemented to absorb these outgoing waves without reflection. Although many types of hybrid CFD methods exist today, combining a variety of different numerical methods, the blending in the present work of a compressible vortex particle method with an Euler solver is the first of its kind.

In Chapter 5, the verification and validation of HEPM was performed. Verification studies were completed in order to ensure the convergence of the numerical method, as well as to qualitatively examine the usefulness of HEPM for the intended rotorcraft applications. Firstly, the convergence of HEPM was examined for steady flow over a NACA0012 airfoil at 0 degrees incidence for low and high Mach numbers. The high 
Mach number case included shock waves symmetrically located on the upper and lower surface of the airfoil. The following observations were made:

- 2-3 iterations between the Euler solver and CVPM are required at each timestep for both flow speeds;

- steady conditions are maintained for all time at both flow speeds;

- an Euler domain extent of 1 chord length from the airfoil is expected to contain even the strongest shocks encountered by the advancing blades for a rotorcraft in a forward flight configuration.

Secondly, an unsteady rapidly pitching airfoil was examined at a moderate Mach number. The following observations were made:

- lift results converged by the 6th cycle;

- good agreement between the converged lift hysteresis as predicted by HEPM and the reference Euler solution;

- smooth transition of particle mesh variables $(\rho, \omega, \delta)$ as they pass from the Euler domain into the particle method domain;

- HEPM is virtually free of the numerical dissipation of vorticity, subject only to the numerical dissipation occurring in the 1 chord length of Euler domain trailing the airfoil. 
It was noted that the particularly long lift convergence time was probably due to the extremely aggressive nature of the pitch speed.

Validation of HEPM was done in comparison with two experimental data sets from the AGARD Compendium of Unsteady Aerodynamic Measurements which feature timescales comparable to rotorcraft applications. Firstly, a ramp-up pitching NACA0012 airfoil was examined with the following results:

- comparisons with experiment became less accurate at higher angles of attack due to the inviscid nature of HEPM;

- lift coefficients were correctly predicted at lower angles of attack;

- good prediction of pressure distributions at lower angles of attack.

Secondly, a sinusoidally pitching NACA0012 airfoil at flow speeds allowing the intermittent presence of shock waves over each cycle. The following observations were made:

- lift results converged by the 3rd cycle;

- good agreement between the converged normal force hysteresis;

- excellent prediction of airfoil surface pressure distributions including accurate shock capturing. 
Finally, HEPM was implemented for airfoil-vortex interaction (AVI) studies. The AVI phenomena is a unique issue in rotorcraft applications, whose capturing is essential to obtain an accurate representation of the rotor aerodynamics. A literature review revealed that transonic rotorcraft AVI studies had never been published. This could be due to the fact that a single rotor configuration in forward flight should not experience significant AVI on the advancing blades undergoing transonic flow conditions. However, in order to increase rotorcraft forward flight speeds, companies such as Sikorsky are investigating co-axial, counter-rotating main rotor disks. In this type of dual rotor configuration, there will certainly be significant AVI in forward flight, as the helical tip vortex from the upper rotor disk interacts with the lower rotor disks. HEPM is ideally suited for this type of simulation. As such, HEPM AVI simulations were run at low and high Mach numbers and various impinging vortex miss distances. As well as predicting the expected trends, the following improvements over conventional grid-based methods were observed:

- the vortex did not wander as it approached the airfoil;

- the vortex did not dissipate as it approached the airfoil;

- empirically-tuned constants were not required as with vorticity-confinement and Large-Eddy Simulation (LES) grid-based methods;

- results were obtained in a timely manner ( $<5$ hours) as compared with other 
AVI studies which can take weeks to run.

Verification and validation results with HEPM confirmed that the aims of this work have been met. The development of the novel hybrid method was successful, the numerical method converges and simulation results are in good agreement with experiment. The method eliminates the need for complex meshing strategies and features exact treatment of surface boundary conditions, high resolution of flow details surrounding the airfoil and numerical dissipation-free convection of vorticity in the far-field. In addition, this unique code proved successful in the advanced application of rotorcraft transonic AVI.

\subsection{Recommendations}

Future recommendations for advancements of HEPM can be categorized as shortterm and long-term advancements. Short-term advancements should focus on the improvement of the methods currently in place.

Firstly, the time integration scheme of the Hamilton-Jacobi equation should be examined. Unsteady simulations are currently limited by the CFL limitations of this explicit scheme. Also with regards to the Hamilton-Jacobi equation, it is important to note the isentropic flow assumption in its derivation. Clearly when shocks are present, this condition is not satisfied exactly. Although the error is expected to be small, it would be interesting to investigate and correct it through the addition of a 
source term.

Secondly, it is necessary to implement FFT solver for orthogonal, but variable mesh densities. This would allow higher particle mesh densities around the airfoil and in regions of high vorticity in order to better resolve the flowfield and improve AVI calculations.

Long-term advancements can focus in two directions. Firstly, the development of a viscous compressible vortex particle method combined with the hybrid coupling with a Navier-Stokes solver, and secondly, the extension of the method to 3-D. A viscous formulation for the compressible vortex particle method is obtained by following the process in section 2.3, using the Navier-Stokes equations instead of the Euler equations. This process will lead to the inclusion of viscosity in the pseudopressure equation, which is subsequently inserted into the Hamilton-Jacobi equation. This couples viscosity with compressibility. Vorticity is coupled to viscosity with the solution of the viscous vorticity transport equation in a Lagrangian manner. This can be done through any of the viscous schemes described in section 1.2.2.

Extension of the method to 3-D involves consideration of two aspects in particular. The particle method is adaptable but the vorticity transport equation must be extended to include the vortex tilting/stretching term. Although fast summation algorithms are implemented in 2-D, a 3-D formulation will require parallelization for efficient computation. The Euler/Navier-Stokes code is already capable, using the 
MPI protocol, however the particle mesh technique must be parallelized. 


\section{References}

[1] "Helicopter fact sheet." from the American Helicopter Society website: www.vtol.org/helifact.html (March 2009).

[2] W. Wilkie, Anisotropic Piezoelectric Twist Actuation of Helicopter Rotor Blades: Aeroelastic Analysis and Design Optimization. PhD thesis, Department of Aerospace Engineering Studies, University of Colorado, 1997.

[3] F. Nitzsche, D. Feszty, D. Waechter, E. Bianchi, S. Voutsinas, M. Gennaretti, G. Coppotelli, and G. Ghiringelli, "The SHARCS project: Smart Hybrid Active Rotor Control System for noise and vibration attenuation of helicopter rotor blades," in 31st European Rotorcraft Forum, (Florence, Italy), pp. 52-1-52-15, Sep 2005.

[4] G. Davis, D. Feszty, and F. Nitzsche, "Trailing edge flow control for the mitigation of dynamic stall effects," in 31st European Rotorcraft Forum, no. 053, (Florence, Italy), September 2005. 
[5] M. Cha, "Feasibility study and design of an actively controlled tip for helicopter rotor blades," Master's thesis, Carleton University, Ottawa, Ontario, Canada, 2007.

[6] F. Nitzsche, D. Feszty, K. Khomutov, B. Lynch, A. Mander, and F. Ulker, "Design and instrumentation of the SHARCS scaled rotor with three independent control systems," in 64th Annual Forum of the American Helicopter Society, (Montreal, Canada), Apr 2008.

[7] J. Leishman, "Principles of helicopter aerodynamics," in Cambridge University Press (C. A. Series, ed.), (New York, USA), 2000.

[8] S. Drzewiecki, "Du choix des elements determinant des helices propulsives permettant leur facile comparaison entre elles," Bulletin de l'Association Technique Maritime, no. 12, 1901.

[9] A. Landgrebe, "The wake geometry of a hovering rotor and its influence on rotor performance," in 28th Annual Forum of the American Helicopter Society, (Washington, DC), May 1972.

[10] J. Kocurek and J. Tangler, "A prescribed wake lifting surface hover performance analysis," Journal of the American Helicopter Socitey, vol. 21, pp. 24-35, 1976.

[11] R. Coleman, A. Feingold, and C. Stenpin, "Evaluation of the induced velocity fields of an idealized helicopter rotor," NACA ARR L5E10, 1945. 
[12] T. Beddoes, "A wake model for high resolution airloads," in 2nd International Conference on Basic Rotorcraft Research, Triangle Park, North Carolina, 1985.

[13] D. Lee, "Numerical prediction of rotor tip-vortex roll-up in axial flights by using a time-marching free-wake method," in Vortex Methods, pp. 177-187, Elsevier Science Publishers, 2000.

[14] S. Voutsinas, "Vortex methods in aeronautics: How to make things work," International Journal of Computational Fluid Dynamics, vol. 20, no. 1, 2006.

[15] S. Voutsinas, "A GENeral Unsteady Vortex Particle method for solving unsteady flow around multi-component configurations." Internal report: Fluids Section, Department of Mechanical Engineering, National Technical University of Athens, Greece, 1990.

[16] D. G. Triantos and S. Voutsinas, "High-resolution aerodynamic analysis of full helicopter configurations," in Proc. 25th European Rotorcraft Forum, (Rome, Italy), pp. 14-16, Sep 1999.

[17] D. Opoku and F. Nitzsche, "Acoustic validation of a new code using particle wake aerodynamics geometrically-exact beam structural dynamics," in Proc. 29th European Rotorcraft Forum, no. 11, (Friedrichshafen, Germany), September 2003 . 
[18] S. McTavish, "Advanced aeroelastic simulations for practical fixed-wing and rotary wing applications," Master's thesis, Carleton University, Ottawa, Ontario, Canada, September 2008.

[19] M. Berkman, L. Sankar, C. Berezin, and M. Torok, "A Navier-Stokes/full potential/free wake method for advancing multi-bladed rotors," in 53rd Annual Forum of the American Helicopter Society, (Virginia Beach, Virginia), American Helicopter Society, May 1997.

[20] M. Moulton, Y. Wenren, and F. Caradonna, "Free-wake hover flow prediction with a hybrid potential/Navier-Stokes solver," in 55th Annual Forum of the American Helicopter Society, (Monteal, Quebec, Canada), May 1999.

[21] M. Dietz, M. Kessler, E. Kramer, and S. Wagner, "Tip vortex conservation on a helicopter main rotor using vortex-adapted Chimera grids," AIAA Journal, vol. 45 , no. 8 , pp. 2062-2074, 2007.

[22] M. Mamou and M. Khalid, "Time-accurate flow simulations past helicopter rotors in hover and forward flight conditions using Chimera grid technique," Canadian Aeronautics and Space Journal, vol. 52, no. 4, pp. 135-148, 2006.

[23] J. Sides, K. Pahlke, and M. Costes, "Numerical simulation of flows around helicopters," in 1st ONERA-DLR Aerospace Symposium, (Paris, France), 1999. 
[24] H. Nam, Y. Park, and O. Kwon, "Simulation of unsteady rotor-fuselage aerodynamic interaction using unstructured adaptive meshes," Journal of the American Helicopter Society, vol. 51, no. 2, pp. 141-149, 2006.

[25] R. Steigl and G. Barakos, "Sliding mesh algorithm for CFD analysis of helicopter rotor-fuselage aerodynamics," International Journal of Numerical Methods in

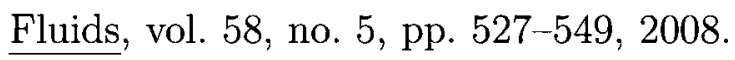

[26] L. Barba, A. Leonard, and C. Allen, "Vortex method with fully meshless implementation for high-Reynolds number flow computations," European Congress on Computational Methods in Applied Sciences and Engineering, July 2004.

[27] G. Cottet and P. Koumoutsakos, Vortex Methods: Theory and Practice. UK: Cambridge University Press, 2000.

[28] L. Greengard and V. Rokhlin, "A fast algorithm for particle simulations," Journal of Computational Physics, vol. 73, no. 2, pp. 325-48, 1987.

[29] B. Cipra, "The best of the 20th century: Editors name top 10 algorithms," SIAM News, vol. 33, no. 4, 2000.

[30] S. Huberson and S. Voutsinas, "Particles and grid," Journal of Computers and Fluids, vol. 31, pp. 607-25, 2002. 
[31] A. Chorin, "Numerical study of slightly viscous flow," Journal of Fluid Mechanics, vol. 57, pp. 785-796, 1973.

[32] L. Barba, A. Leonard, and C. Allen, "Advances in viscous vortex methods - meshless spatial adaptation based on radial basis function interpolation," International Journal for Numerical Methods in Fluids, vol. 47, no. 5, pp. 387$421,2005$.

[33] A. Leonard, "Vortex methods for flow simulation," Journal of Computational Physics, vol. 37, no. 3, pp. 289-335, 1980.

[34] C. Greengard, "Note: The core spreading vortex method approximates the wrong equation," Journal of Computational Physics, vol. 61, no. 2, pp. 345-48, 1985.

[35] L. Rossi, "Resurrecting core spreading vortex methods: a new scheme that is both deterministic and convergent," SIAM Journal on Scientific Computing, vol. 17, no. 2, pp. 370-97, 1996.

[36] P. Degond and S. Mas-Gallic, "The weighted particle method for convectiondiffusion equations part 1: The case of an isotropic viscosity," Mathematics of Computation, vol. 53, no. 188, pp. 485-507, 1989.

[37] R. Meske, A Vortex-In-Cell Method for Compressible Flow. PhD thesis, Imperial College of Science and Technology, London,UK, 1994. 
[38] S. Mas-Gallic, M. Louaked, and O. Pironneau, "A particle in cell method for 2d compressible Euler equations," in Vortex Flows and Related Methods, vol. 395, pp. 373-387, Kluwer Series Mathematical and Physical Sciences, 1993.

[39] J. Eldredge, A Dilating Vortex Particle Method for Compressible Flow with Applications to Aeroacoustics. PhD thesis, California Institute of Technology, July 2002.

[40] M. Ould-Salihi, G. Cottet, and M. E. Hamraoui, "Blending finite-difference and vortex methods for incompressible flow computations," Society for Industrial and Applied Mathematics, pp. 1655-1674, December 2000.

[41] J. Guermond, S. Huberson, and W. Shen, "Simulation of 2D external viscous flows by means of a domain decomposition method," Journal of Computational Physics, no. 108, pp. 343-352, 1993.

[42] O. Hald, "Convergence of vortex methods for Euler equations," SIAM Journal of Numerical Analysis, vol. 16, pp. 726-755, 1979.

[43] J. Beale and A. Majda, "Vortex methods II: Higher order accuracy in two and three dimensions," Mathematics and Computations, vol. 39, no. 159, pp. 29-52, 1982.

[44] P. Raviart, "An analysis of particle methods," in Numerical Methods in Fluid Mechanics of Lecture Notes in Mathematics, pp. 243-324, 1983. 
[45] C. Anderson and C. Greengard, "On vortex methods," SIAM Journal of Numerical Analysis, vol. 22, no. 3, pp. 413-440, 1985.

[46] R. Boisvert, "A fourth order accurate fast direct method for the Helmholtz equation," in Elliptic Problem Solvers, (Orlando, Fla), pp. 35-44, Academic Press, 1984.

[47] R. Gingold and J. Monaghan, "Shock simulation by the particle method SPH," Journal of Computational Physics, vol. 52, pp. 374-389, 1983.

[48] S. Osher and C. Shu, "Higher order essentially nonoscillatory schemes for Hamilton-Jacobi equations," SIAM Journal of Numerical Analysis, vol. 28, no. 4, pp. 907-922, 1991.

[49] K. Badcock, M. Woodgate, F. Cantariti, and B. Richards, "Solution of the unsteady Euler equations in three dimensions using a fully unfactored method." Aerospace Engineering Report 9909, University of Glasgow, 1999.

[50] K. Badcock, B. Richards, and M. Woodgate, "Elements of computational fluid dynamics on block structured grids using implicit solvers," Progress in Aerospace

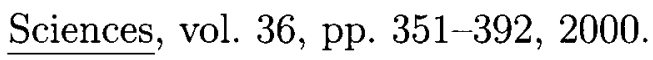

[51] M. Woodgate, K. Badcock, A. Rampurawala, and B. Richards, "Aeroelastic calculations for the Hawk aircraft using the Euler equations." Aerospace Engineering Report 0313, University of Glasgow, 2003. 
[52] R. V. Leer, "Towards the ultimate conservative scheme: A second order sequel to Godunov's method," Journal of Computational Physics, vol. 32, pp. 101-136, 1979.

[53] G. V. Albada, R. V. Leer, and W. Roberts, "A comparative study of computational methods in cosmic gas dynamics," Astronomy and Astrophysics, vol. 108, p. 76, 1982.

[54] A. Jameson, "Time dependent calculations using multigrid with applications to unsteady flows past airfoils and wings," AIAA Paper 91-1596, 1991.

[55] J. Freund, "Proposed inflow/outflow boundary condition for direct computation of aerodynamic sound," AIAA Journal, vol. 35, no. 4, pp. 740-742, 1997.

[56] R. Landon, "N0012, oscillatory and transient pitching," AGARD Report No.707, pp. 3.1-3.25, 1982 .

[57] A. Wong, "A study on the use of Volterra integrals for the identification of rotor blade-vortex interactions," Master's thesis, Carleton University, Ottawa, Ontario, Canada, 2003.

[58] F. Falissard, A. Lerat, and J. Sides, "Computation of airfoil-vortex interaction using a vorticity-preserving scheme," AIAA Journal, vol. 46, no. 7, pp. 1614$1623,2008$. 
[59] R. Morvant, K. Badcock, G. Barakos, and B. Richards, "Airfoil-vortex interaction using the compressible vorticity-confinement method," AIAA Journal, vol. 43 , no. 1, pp. 63-75, 2005.

[60] S. Lee and D. Bershader, "Head-on blade-vortex interaction," AIAA Journal, vol. 32 , no. 1, pp. 16-22, 1994.

[61] W. Splettstoesser, "The HELINOISE aeroacoustic rotor test in the DNW - test documentation and representative results," in DLR-Mitt, (DLR Braunschweig Germany), 93-09 1993.

[62] A. Harten and S. Osher, "Uniformly high-order accurate nonoscillatory schemes," SIAM Journal on Numerical Analysis, vol. 24, no. 2, pp. 279-309, 1987.

[63] A. Harten, B. Engquist, S. Osher, and S. Chakravarthy, "Uniformly high-order essentially nonoscillatory schemes," Journal of Computational Physics, vol. 71, no. 2, pp. 231-303, 1987.

[64] C. Shu and S. Osher, "Efficient implementation of essentially nonoscillatory shockcapturing schemes," Journal of Computational Physics, vol. 77, no. 2, pp. $439-471,1988$.

[65] K. Badcock, "How to run a job." University of Glasgow Report: TN02-001, 1990. 


\section{Appendix A}

\section{ENO Scheme Formulation}

\section{A.1 Introduction}

The evolution of the flow compressibility involves the advancement of a scalar potential, $\phi$, given by equation (2.37). This equation has the form of a well known Hamilton-Jacobi class of equations, which are analogous to a hyperbolic conservation law integrated once:

$$
\begin{aligned}
\frac{\partial \phi}{\partial t}+H(\nabla \phi) & =0 \\
\phi(x, 0) & =\phi_{0}(x)
\end{aligned}
$$

where $H$ is the Hamiltonian. In the potential field of a compressible flow, one would expect discontinuities in $\phi$ at shock locations and across solid boundaries. As a result, the use of a central finite-difference scheme to disretize equation (A.1) would result in spurious solution oscillations in the regions surrounding the discontinuities. To avoid these oscillations, an Essentially Non-Oscillatory (ENO) scheme is implemented which uses an adaptive polynomial interpolation stencil, automatically 
obtaining information from the locally smoothest regions. This yields a high-order non-oscillatory approximation for piecewise smooth functions. The following formulation follows Osher and Shu [48], who successfully implemented ENO schemes to solve various convex and non-convex problems in 1 and 2-dimensions. The reader is also referred to further discussions on ENO schemes in [62], [63] and [64].

\section{A.2 Interpolating Polynomials}

Given values $f\left(x_{j}\right)$ at discrete nodes $x_{j}$, we associate an $r^{\text {th }}$ degree polynomial, $P_{j+1 / 2}^{f, r}$ to each interval $\left[x_{j}, x_{j+1}\right]$ constructed inductively as follows:

1. $P_{j+1 / 2}^{f, 1}(x)=f\left[x_{j}, x_{j+1}\right]\left(x-x_{j}\right), k_{\min }=j$

2. If $k_{\min }^{(l-1)}$ and $P_{j+1 / 2}^{f, l-1}(x)$ are both defined, then let

$$
\begin{aligned}
a^{(l)} & =f\left[x_{k_{\text {min }}^{l-1}}, \cdots, x_{k_{\text {min }}^{l-1}+l}\right] \\
b^{(l)} & =f\left[x_{k_{\text {min }}^{l-1}-1}, \cdots, x_{k_{m i n}^{l-1}+l-1}\right]
\end{aligned}
$$

3. If $\left|a^{(l)}\right| \geq\left|b^{(l)}\right|$, then $c^{(l)}=b^{(l)}, k_{\text {min }}^{(l)}=k_{\text {min }}^{(l-1)}-1$, otherwise $c^{(l)}=a^{(l)}, k_{\text {min }}^{(l)}=$ $k_{\min }^{(l-1)}$

4. $P_{j+1 / 2}^{f, l}(x)=P_{j+1 / 2}^{f, l-1}(x)+c^{(l)} \prod_{i=k_{m i n}^{(l-1)}}^{k_{m i n}^{(l-1)}+l-1}\left(x-x_{i}\right)$

where $f[\cdot, \cdots, \cdot]$ represents the Newtonian divided difference given by:

$$
f\left[x_{0}, x_{1}, \cdots, x_{n}\right]=\frac{f\left[x_{n}, x_{n-1}, \cdots, x_{1}\right]-f\left[x_{0}, x_{1}, \cdots, x_{n-1}\right]}{x_{n}-x_{0}}
$$




\section{A.3 Scheme Construction}

Osher and Shu insert the Hamiltonian, $H$, into a number of monotonic fluxes including Lax-Freidrichs, Godunov, Local Lax-Freidrichs (LLF) and Roe's scheme with LLF entropy correction. In this work the latter is implemented and is described as:

$$
\hat{H}^{R F}\left(u^{+}, u^{-}, v^{+}, v^{-}\right)= \begin{cases}H\left(u^{*}, v^{*}\right) & \text { - if } H_{1}(u, v) \text { and } H_{2}(u, v) \\ & \text { do not change signs in } \\ H\left(\frac{u^{+}+u^{-}}{2}, v^{*}\right)- & u \epsilon I\left(u^{-}, u^{+}\right), v \epsilon I\left(v^{-}, v^{+}\right) \\ \frac{1}{2} \alpha^{x}\left(u^{+}, u^{-}\right)\left(u^{+}-u^{-}\right) & \text {- otherwise and if } H_{2}(u, v) \\ H\left(u^{*}, \frac{v^{+}+v^{-}}{2}\right)- & A \leq u \leq B, v \epsilon I\left(v^{-}, v^{+}\right) \\ \frac{1}{2} \alpha^{y}\left(v^{+}, v^{-}\right)\left(v^{+}-v^{-}\right) & \text {- otherwise and if } H_{1}(u, v) \\ \hat{H}^{L L F}\left(u^{+}, u^{-}, v^{+}, v^{-}\right) & \text {- otherwise change signs in }\end{cases}
$$

where $u^{*}, v^{*}$ are defined by upwinding:

$$
u^{*}=\left\{\begin{array}{ll}
u^{+} & \text {if } H_{1}(u, v) \leq 0, \\
u^{-} & \text {if } H_{1}(u, v) \geq 0,
\end{array} \quad v^{*}= \begin{cases}v^{+} & \text {if } H_{2}(u, v) \leq 0 \\
v^{-} & \text {if } H_{2}(u, v) \geq 0\end{cases}\right.
$$

$\alpha^{x}, \alpha^{y}$ are defined by:

$$
\begin{array}{ccc}
\alpha^{x}\left(u^{+}, u^{-}\right)= & \max _{u \in I\left(u^{-}, u^{+}\right)}\left|H_{1}(u, v)\right| \\
& C \leq v \leq D \\
\alpha^{y}\left(v^{+}, v^{-}\right)= & \max _{\substack{A \leq u \leq B\\
}}\left|H_{2}(u, v)\right| \\
& v \in I\left(v^{-}, v^{+}\right)
\end{array}
$$


and $H^{L L F}$ is the LLF monotonic flux defined as:

$$
\begin{aligned}
\hat{H}^{L L F}\left(u^{+}, u^{-}, v^{+}, v^{-}\right) & =H\left(\frac{u^{+}+u^{-}}{2}, \frac{v^{+}+v^{-}}{2}\right)- \\
& \frac{1}{2} \alpha^{x}\left(u^{+}, u^{-}\right)\left(u^{+}-u^{-}\right)-\frac{1}{2} \alpha^{y}\left(v^{+}, v^{-}\right)\left(v^{+}-v^{-}\right)
\end{aligned}
$$

\section{A.4 Time Integration}

The velocity at any node $i j$ is taken by the derivative of the interpolating polynomial by:

$$
u_{i j}^{ \pm}=\frac{d}{d x} P_{i \pm \frac{1}{2}, j}^{\phi, r}\left(x_{i}\right)
$$

and similarly for $v_{i j}^{ \pm}$. Let

$$
L_{i j}=-\Delta t \hat{H}\left(u_{i j}^{+}, u_{i j}^{-}, v_{i j}^{+}, v_{i j}^{-}\right)
$$

Time integration is done with the following Runge-Kutta type procedure:

$$
\begin{gathered}
\phi_{i j}^{(k)}=\sum_{l=0}^{k-1}\left[\alpha_{k l} \phi_{i j}^{(l)}+\beta_{k l} L_{i j}^{(l)}\right], \quad k=1,2, \ldots r \\
\phi_{i j}^{0}=\phi_{i j}^{n}, \quad \phi_{i j}^{r}=\phi_{i j}^{n+1}
\end{gathered}
$$

\begin{tabular}{|c|c|c|c|}
\hline Order & $\alpha_{k l}$ & $\beta_{k l}$ & $C_{r}$ \\
\hline 1 & 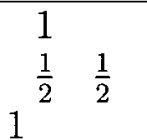 & 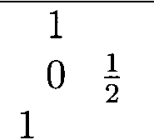 & 1 \\
\hline 2 & $\begin{array}{lll}\frac{3}{4} & \frac{1}{4} & \\
\frac{1}{3} & 0 & \frac{2}{3}\end{array}$ & $\begin{array}{lll}0 & \frac{1}{4} & \\
0 & 0 & \frac{2}{3} \\
\end{array}$ & 1 \\
\hline
\end{tabular}

Table A.1: TVD Runge-Kutta coefficients

The Runge-Kutta coefficients are found from Table (A.1) and the method is proven Time-Variation Diminishing (TVD) under the following CFL condition: 


$$
\lambda=\frac{\Delta t}{\Delta x} \leq C_{r} \lambda_{0}
$$

\section{A.5 1-D Validation}

In one dimensions we solve: $\phi_{t}+H\left(\phi_{x}\right)=0$

\section{A.5.1 Case 1: Non-convex $H$}

The Hamiltonian is given by $H(u)=-\cos (u)$ solved on domain $-1 \leq x \leq 1$ with initial conditions $\phi(x, 0)=-\cos \pi x$. Figure (A.1) displays the various monotonic fluxes implemented.

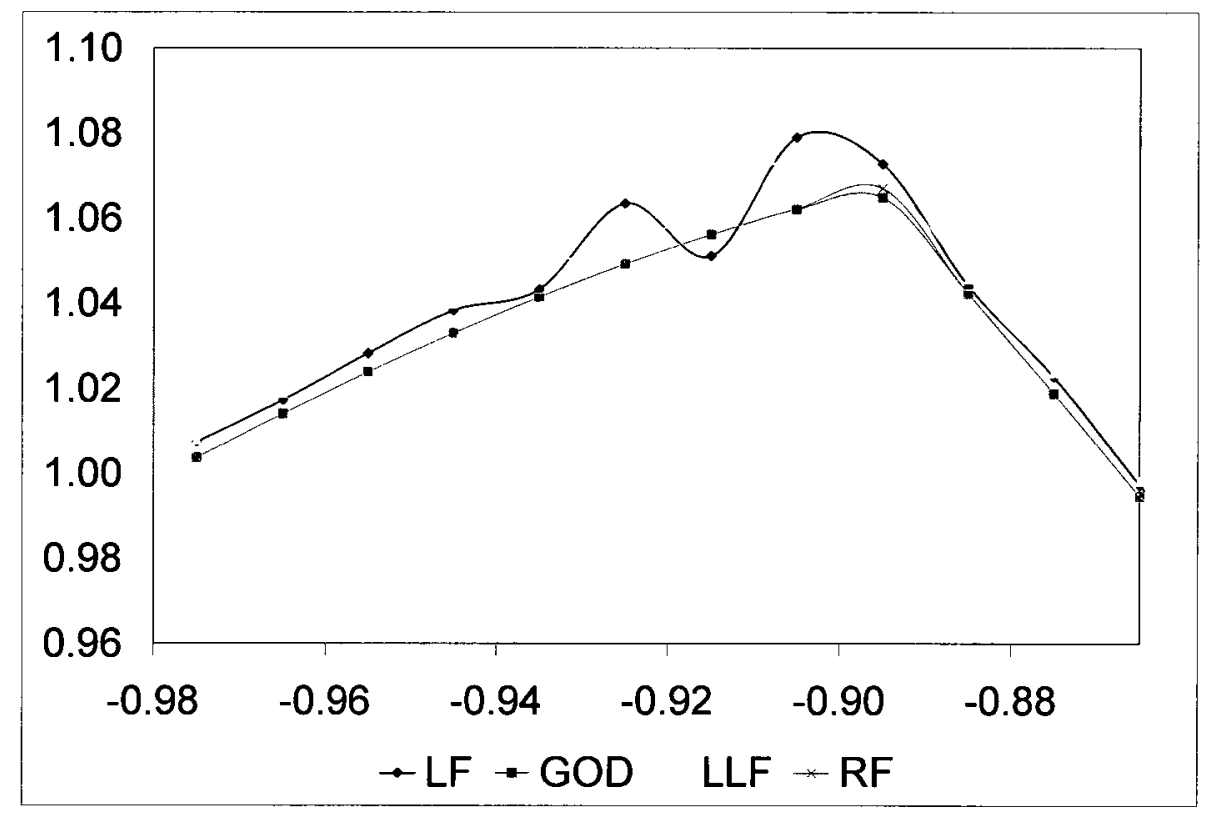

Figure A.1: Discontinuity capturing for 1-D non-convex problem using various monotonic fluxes: Lax-Freidrichs (LF), Godunov (GOD), Local Lax-Freidrichs (LLF) and Roe's flux with LLF entropy correction $(\mathrm{RF}) .\left(t=1.5 / \pi^{2}\right)$ 
It is found that the Roe's scheme with the LLF entropy calculation is the most effective. Figure (A.2) demonstrates the effectiveness of Roe's scheme in comparison with the exact solution.

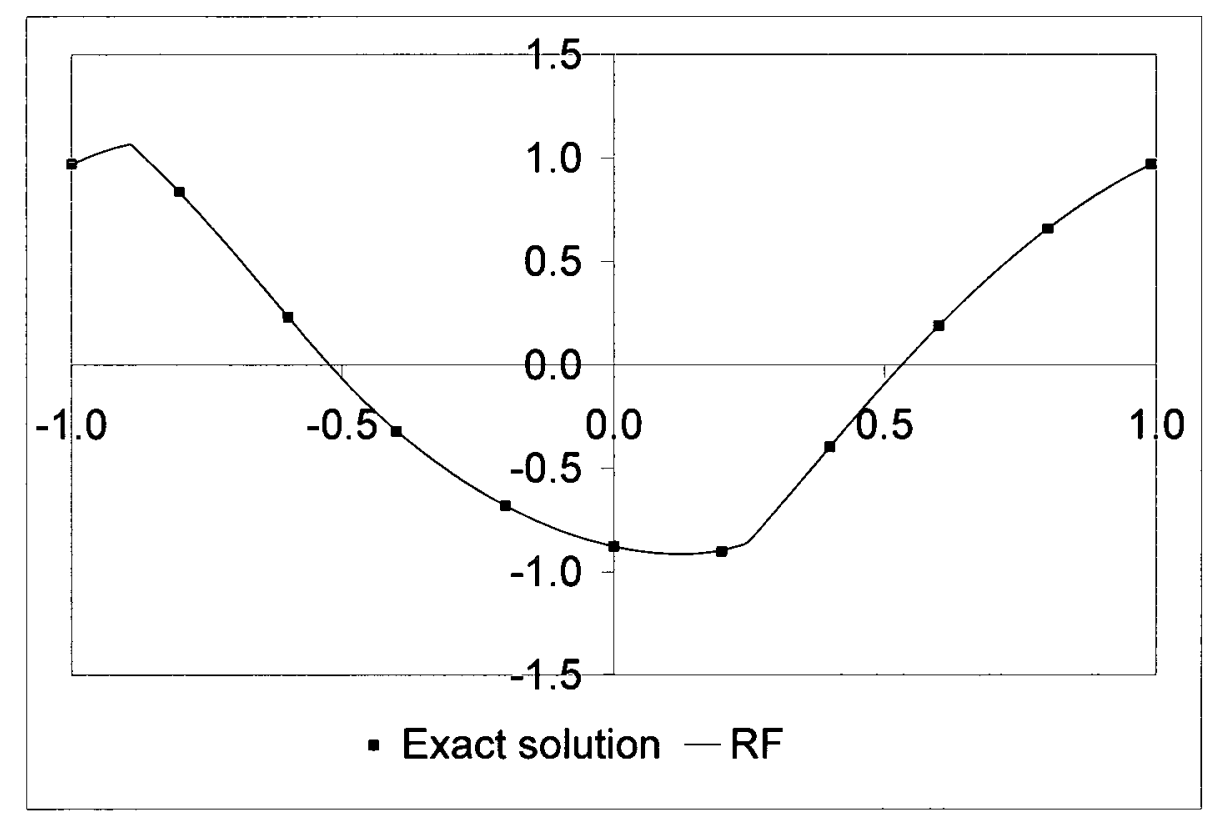

Figure A.2: Comparison of numerical and exact solution to 1-D non-convex problem. Roe's flux with LLF entropy correction implemented. $\left(t=1.5 / \pi^{2}\right)$ 


\section{A.5.2 Case 2: Burger's equation (convex $\mathrm{H}$ )}

The Hamiltonian is given by $H(u)=u^{2} / 2$ solved on domain $-1 \leq x \leq 1$ with initial conditions $\phi(x, 0)=-\cos \pi x$. Figure (A.3) demonstrates the difference in the various monotonic fluxes in capturing the discontinuity. As before, it is found that the Roe's scheme with the LLF entropy calculation is the most effective. Figure (A.4) demonstrates the effectiveness of Roe's scheme in comparison with the exact solution.

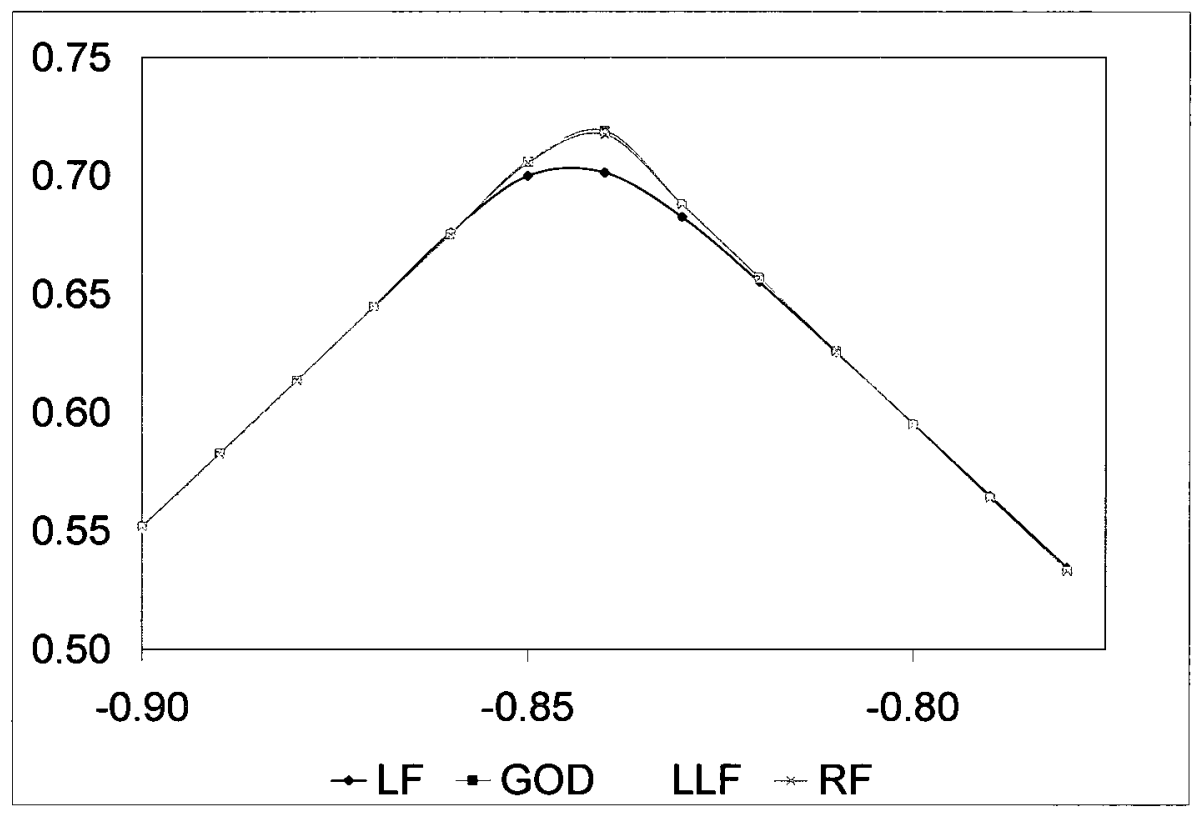

Figure A.3: Discontinuity capturing for Burger's problem using various monotonic fluxes: Lax-Freidrichs (LF), Godunov (GOD), Local Lax-Freidrichs (LLF) and Roe's flux with LLF entropy correction (RF). $\left(t=1.5 / \pi^{2}\right)$ 


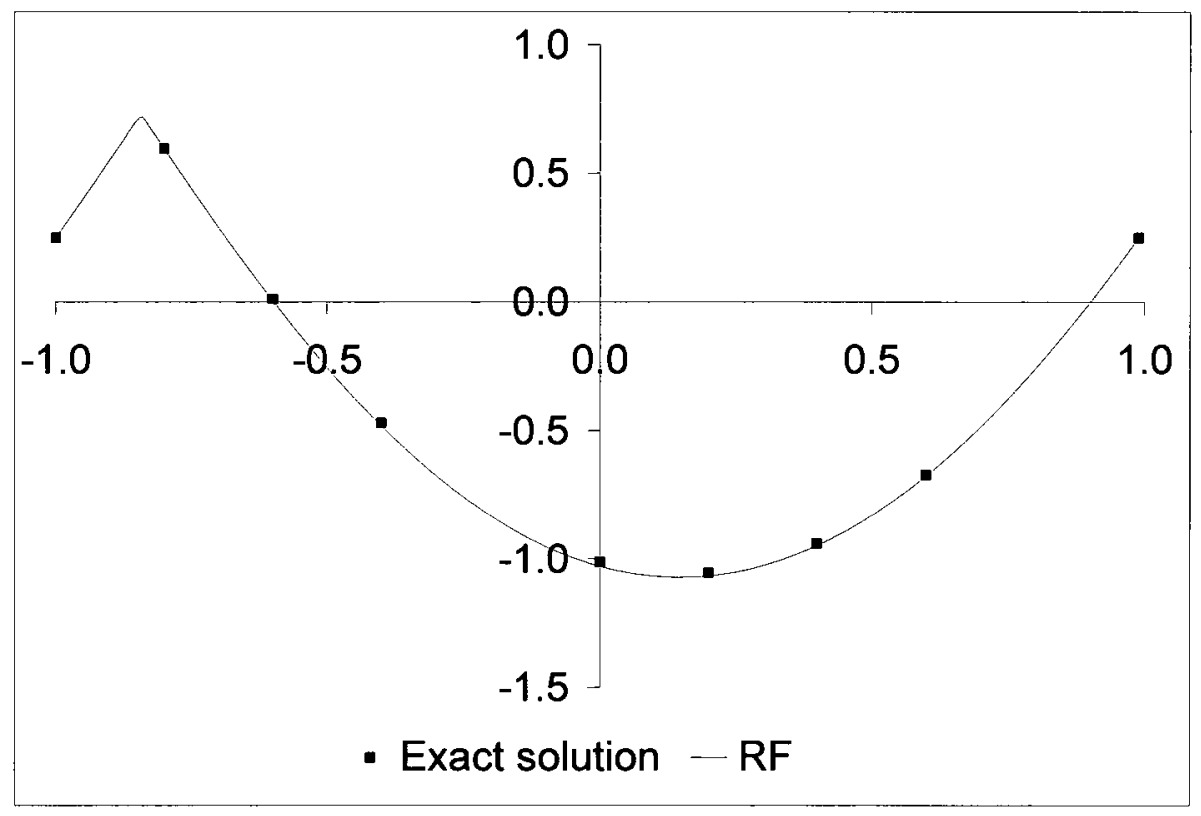

Figure A.4: Comparison of numerical and exact solution to Burger's problem. Roe's flux with LLF entropy correction implemented. $\left(t=1.5 / \pi^{2}\right)$

\section{A.6 2-D Validation}

In one dimensions we solve: $\phi_{t}+H\left(\phi_{x}, \phi y\right)=0$

\section{A.6.1 Case 3: Non-convex $\mathrm{H}$ )}

The Hamiltonian is given by $H(u, v)=(u+v)^{2} / 2$ solved on domain $-2 \leq x \leq 2$ with initial conditions $\phi(x, 0)=-\cos (\pi(x+y) / 2)$. 

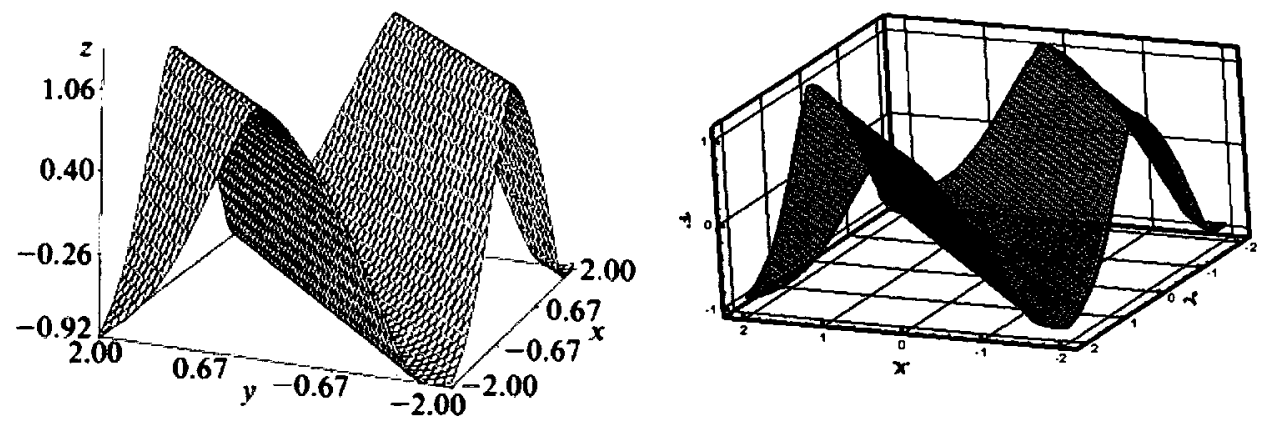

Figure A.5: Comparison of exact (left) and numerical (right) solutions to the 2D nonconvex equation. Numerical solution employs the Roe's scheme with LLF entropy correction. $\left(t=1.5 / \pi^{2}\right)$

\section{A.6.2 Case 4: Burger's equation (convex $H$ )}

The Hamiltonian is given by $H(u, v)=-\cos (u+v)$ solved on domain $-2 \leq x \leq 2$ with initial conditions $\phi(x, 0)=-\cos (\pi(x+y) / 2)$.
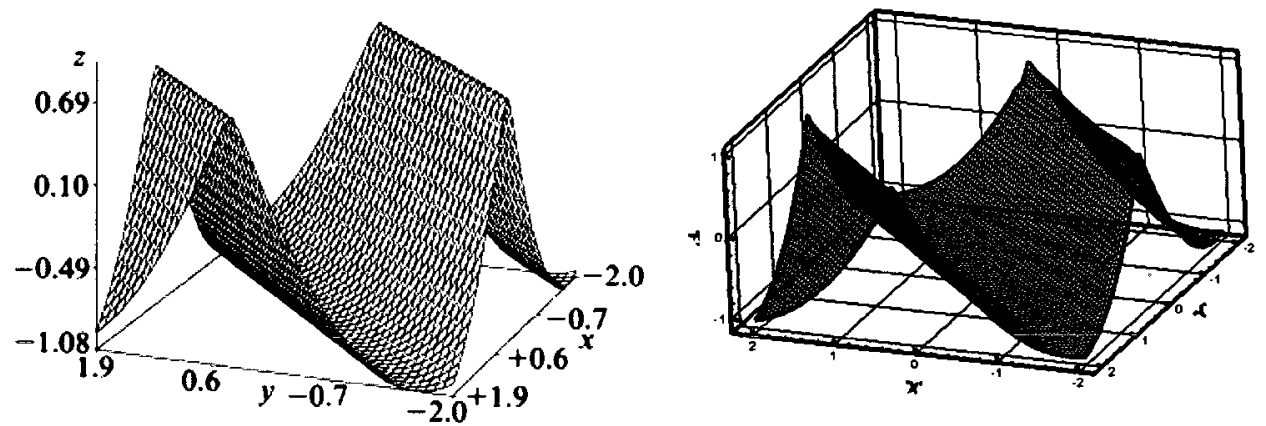

Figure A.6: Comparison of exact (left) and numerical (right) solutions to the 2D Burger's equation. Numerical solution employs the Roe's scheme with LLF entropy correction. $\left(t=1.5 / \pi^{2}\right)$ 


\section{Appendix B}

\section{User Information}

This section contains user information for the HEPM code. Table B.1 lists the code input files, Table B.2 lists code output and Table B.3 lists current scripts for execution of different configurations (reference, steady and unsteady simulations) in addition to the auxiliary utilities. The final section details the approach for compiling and linking this mixed language C-Fortran code.

\section{B.1 Input Files}

Table B.1: HEPM Input Files

\begin{tabular}{ll}
\hline \hline Filename & Description \\
\hline *.cmb.grd & -original full Euler grid geometry \\
*.cmb.hyb & -reduced Hybrid Method Euler grid geometry \\
fine_ptcls.dat & -Particle reference solution (initial guess) \\
GridContraction.DAT & -grid contraction parameters (reference to hybrid) \\
HybridInput.DAT & -Hybrid Method parameters \\
outputtime.dat & -Time array for desired Cp output intervals \\
ref_soln.dat & -Euler reference solution \\
steady_ref & -Euler domain steady flow parameters (reference) \\
steady_hyb & -Euler domain steady flow parameters (hybrid) \\
unsteady & -unsteady motion parameters
\end{tabular}




\section{B.2 Output Files}

Table B.2: HEPM

\begin{tabular}{|c|c|c|}
\hline Filename & Description & Output \\
\hline$*$ *.plt.0000 & -Euler solution at time $\sharp$ & specified \\
\hline *. .surface. 0000 & -Euler solution surface Cp by block & specified \\
\hline *.int & -Euler solution surface integration & continuous \\
\hline agard.dat & -Cp distribution & specified \\
\hline AirfoilMovie.dat & -airfoil geometry animation & specified \\
\hline BoundaryPtsMovie.dat & -boundary cell geometry animation & specified \\
\hline BoundaryValueMovie.dat & -boundary cell value animation & specified \\
\hline DWMovie.dat & -PM solution animation & specified \\
\hline ENORHS.dat & -H-J eqn. RHS terms & last step \\
\hline ENOSIMPLE.dat & -H-J time integration before+after & last step \\
\hline eulerp.dat & -Euler domain particle distribution & last step \\
\hline field_cellcentered.dat & -Euler cell averaged values & last step \\
\hline field_gdruv.dat & -Euler differentiated field & last step \\
\hline hfftphi.dat & -PM scalar potential field & last step \\
\hline hfftpsi.dat & -PM vector potential field & last step \\
\hline pconvect.dat & -particle distribution & last step \\
\hline pmdilatation.dat & -PM dilatation field & last step \\
\hline pm_e_converge.dat & -PM $<->$ Euler convergence history & continuous \\
\hline pmparams.dat & -PM geometry parameters & first step \\
\hline pout.dat & -outer particle distribution & last step \\
\hline project0.dat & -timestep initial PM solution & last step \\
\hline project1.dat & -updated PM solution & last step \\
\hline qcheck.dat & -PM pseudo-pressure, $q$ & last step \\
\hline RUVmovie.dat & -PM $(\rho, u, v)$ animation & specified \\
\hline SurfaceMovie0.dat & -Euler surface disturbance animation & specified \\
\hline SurfaceMovie.dat & -Euler surface velocity animation & specified \\
\hline vcheck0.dat & -PM initial steady velocity field & first step \\
\hline vcheck.dat & -PM velocity field & last step \\
\hline
\end{tabular}




\section{B.3 Script Files}

Table B.3: HEPM Script Files

\begin{tabular}{ll}
\hline \hline Filename & Description \\
\hline runbase_std & -run steady state reference solution \\
runbase_uns & -run unsteady reference solution \\
runhm_std & -run Hybrid Method steady solution \\
runhm_uns & -run Hybrid Method unsteady solution \\
runmotion & -run unsteady file utility \\
runref & -run reference solution for Hybrid Method initial guess \\
runshrink & -run grid reduction utility
\end{tabular}

\section{B.4 Compiling and Linking}

HEPM is a mixed language code written in both FORTRAN77 and C. In general, CVPM routines are written in FORTRAN77 and Euler solver routines are written in C. The procedure for compiling and linking the code is as follows:

1. Compile FORTRAN files with FORTRAN compiler (f77 -g -c *.f)

2. Compile $\mathrm{C}$ files with $\mathrm{C}$ compiler (gcc -g -c *.c)

3. Perform linking off all object files with FORTRAN compiler (f77 -g -o exe * ${ }^{*}$ )

\section{B.5 Simulation Instructions}

The procedure for executing a HEPM simulation can be divided into three main sections: geometry creation, simulation initialization and post-processing. The file structure for the HEPM directory is as follows: 


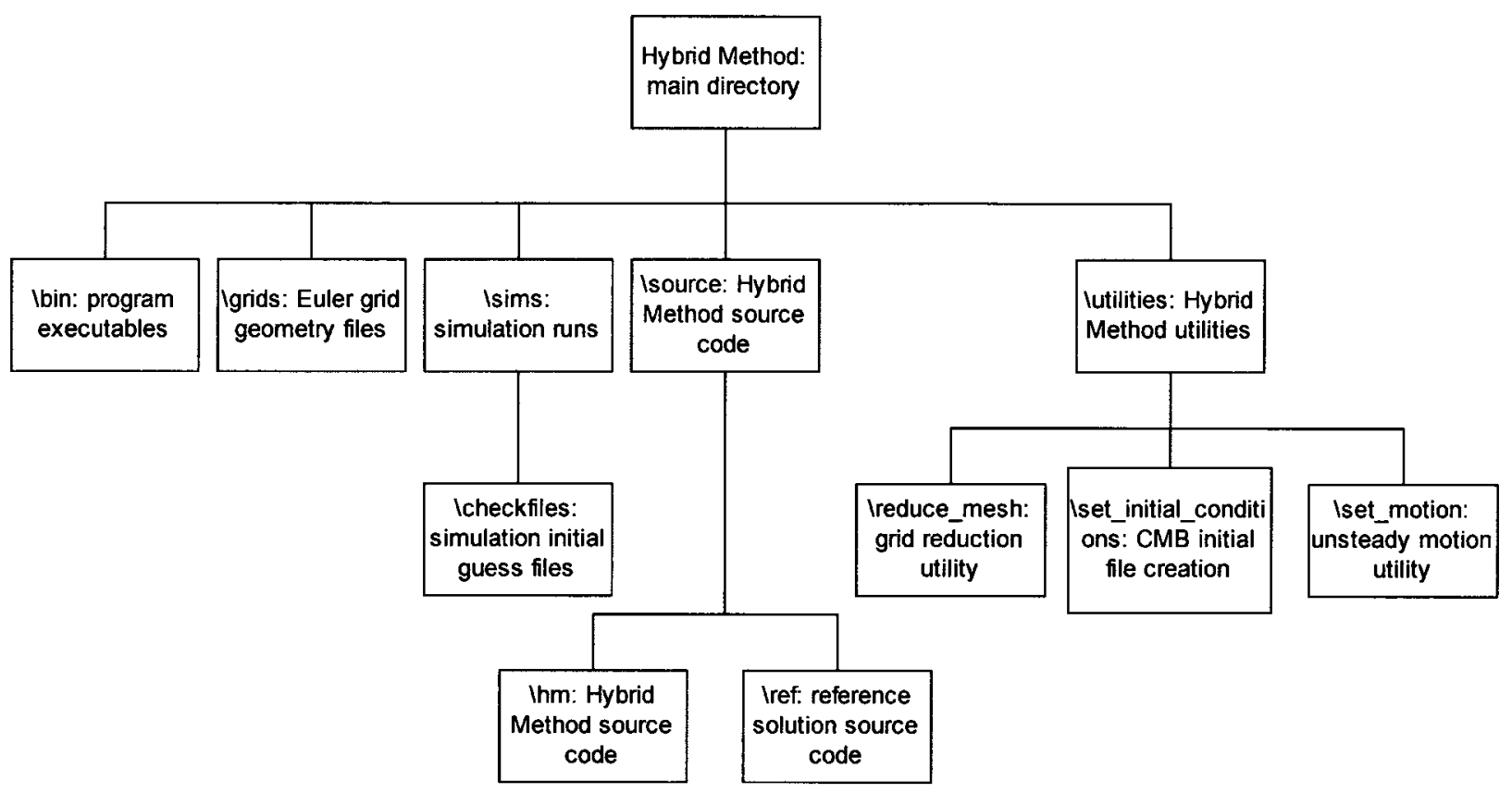

Figure B.1: HEPM directory structure

\section{B.5.1 Geometry Creation}

The first step in the geometry creation is the development of a reference solution grid. This is accomplished with ICEM CFD (current version 10). It is important to consider the following points:

1. The reference grid must extend to at least 10 chord lengths from the airfoil.

2. In order to force the z-component of velocity to zero, it is necessary to place the symmetry planes at $z= \pm 1$.

3. Smooth transition between blocks is necessary to avoid non-physical vorticity 


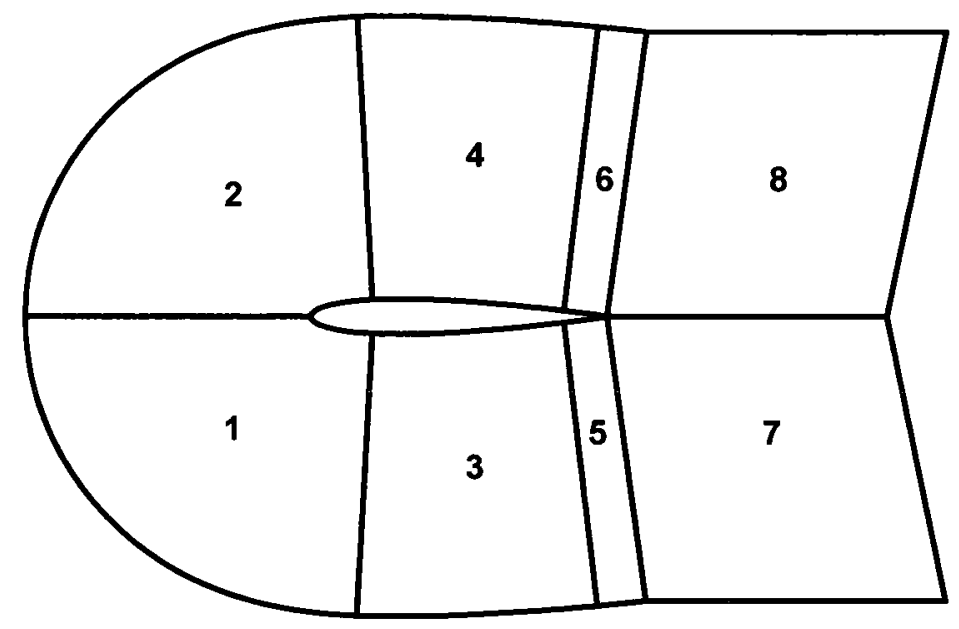

Figure B.2: HEPM block numbering scheme

The output from ICEM CFD is in the form of *.info and *.geo grid topography files. Finally, this is converted to CMB format using the hexa2pmb conversion utility. Directions for the use of this utility are found in [65]. The next step is to reduce to set up the HEPM model. This is done by first reducing the reference grid with the reduce_mesh utility and then encompassing this reduced grid with the particle mesh geometry. The following points are to be considered:

1. The reduced Euler grid must include all regions in which shocks can be expected to form. If no shocks are present, the code still returns good results as long as the Euler grid extends to at least 0.8 chord lengths from the airfoil. There is no maximum extent, but computational restraints should be considered.

2. The particle mesh must extend to at least 1 chord length from the Euler grid 
outer boundary.

3. Particle mesh grid spacing must be uniform (due to FFT solver implemented) and a maximum grid spacing of 0.02 is recommended.

\section{B.5.2 Simulation Execution}

Once the simulation geometry is finalized, simulation execution may be undertaken. It is necessary to first run a reference solution to provide an initial guess. This avoids an "impulse" type start to the numerical method which could affect the convergence. The following command steps are done in the following order:

1. ./runmotion (user prompted creation of unsteady motion file)

2. ./runref (provides initial guess and copies it to / sims/checkfiles directory)

3. ./runhm_std (steady solution, check solution obtained)

4. ./runhm_uns (runs unsteady solution) 


\section{Appendix $\mathrm{C}$}

\section{Code Flow Diagram}

\section{C.1 Main Code Structure}

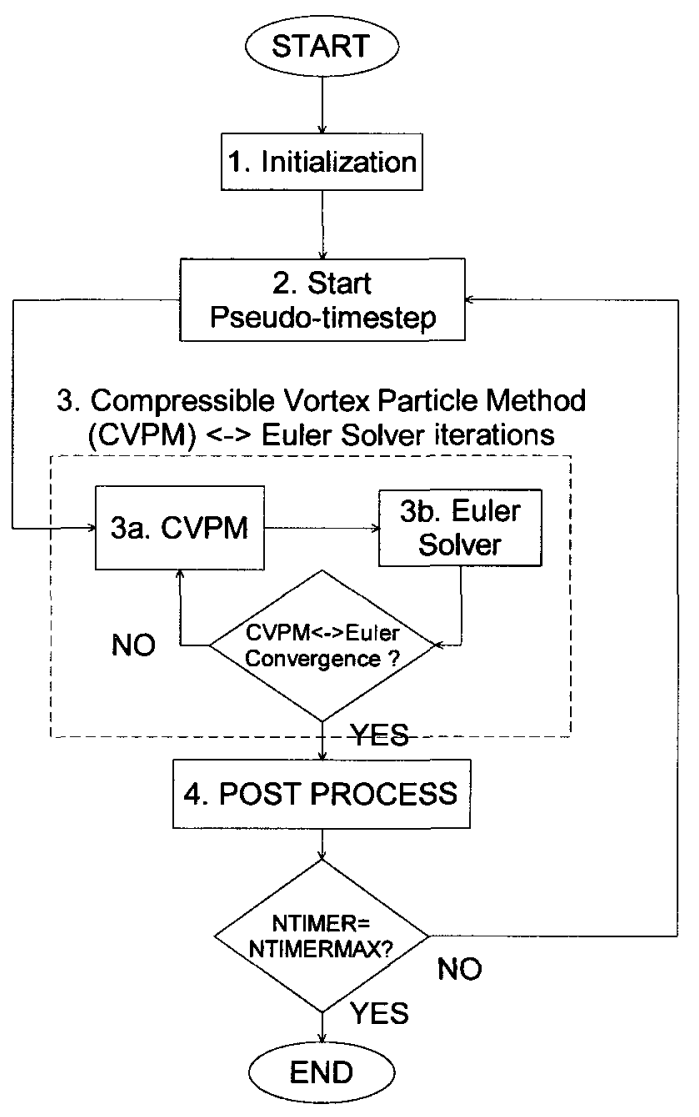




\title{
C.2 Detailed Code Structure
}

\author{
INITIALIZATION (p.1/2)
}

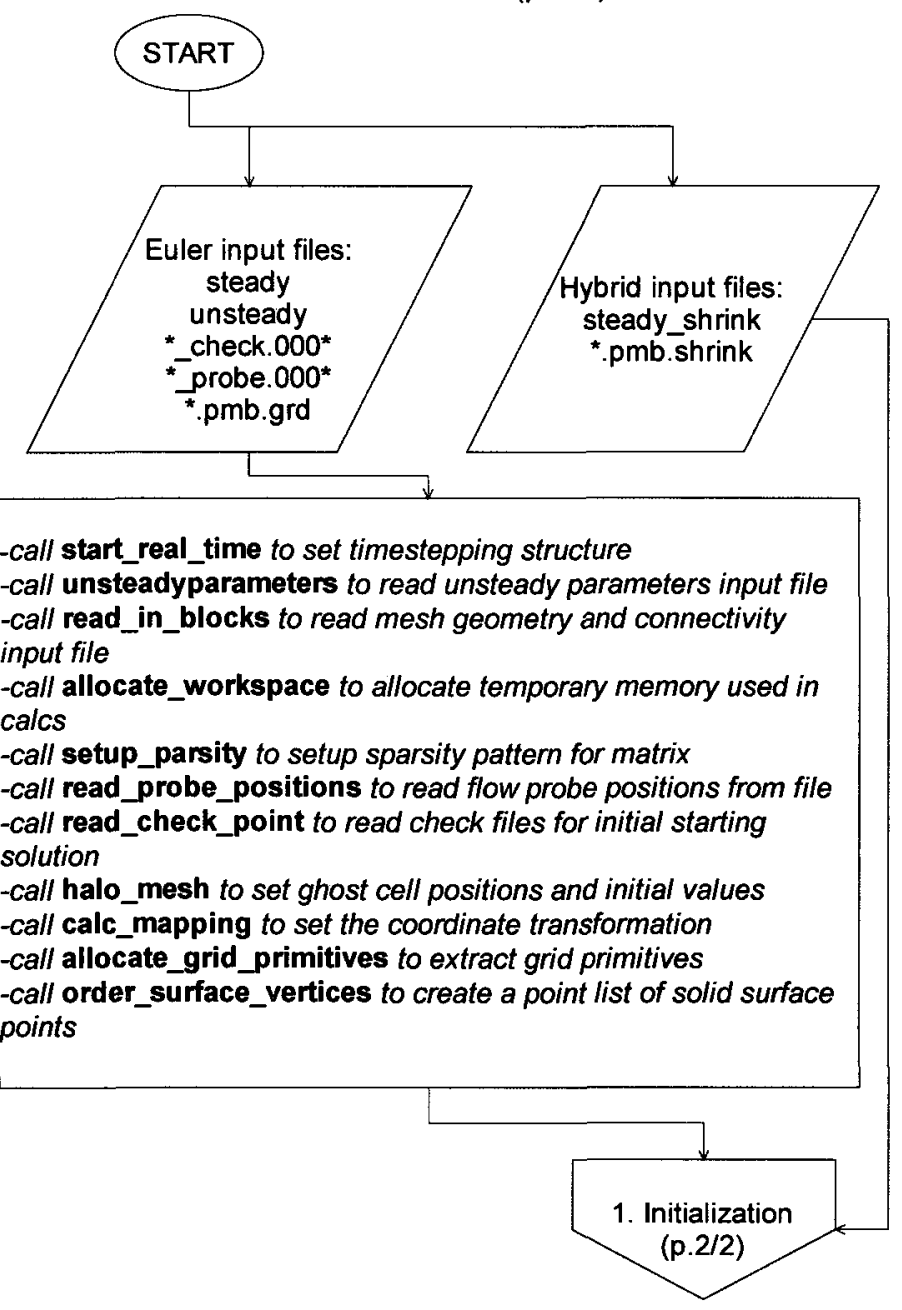


INITIALIZATION (p.2/2)

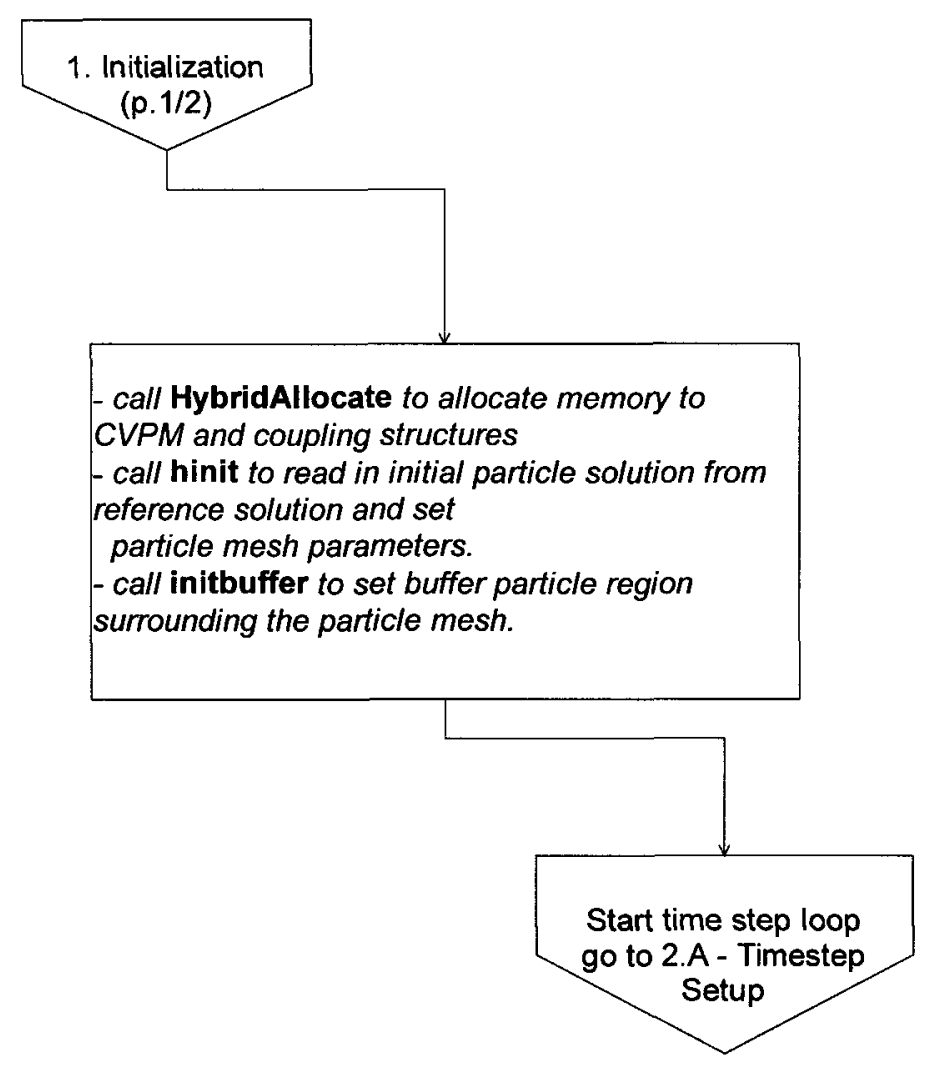




\section{START PSEUDO TIMESTEP}

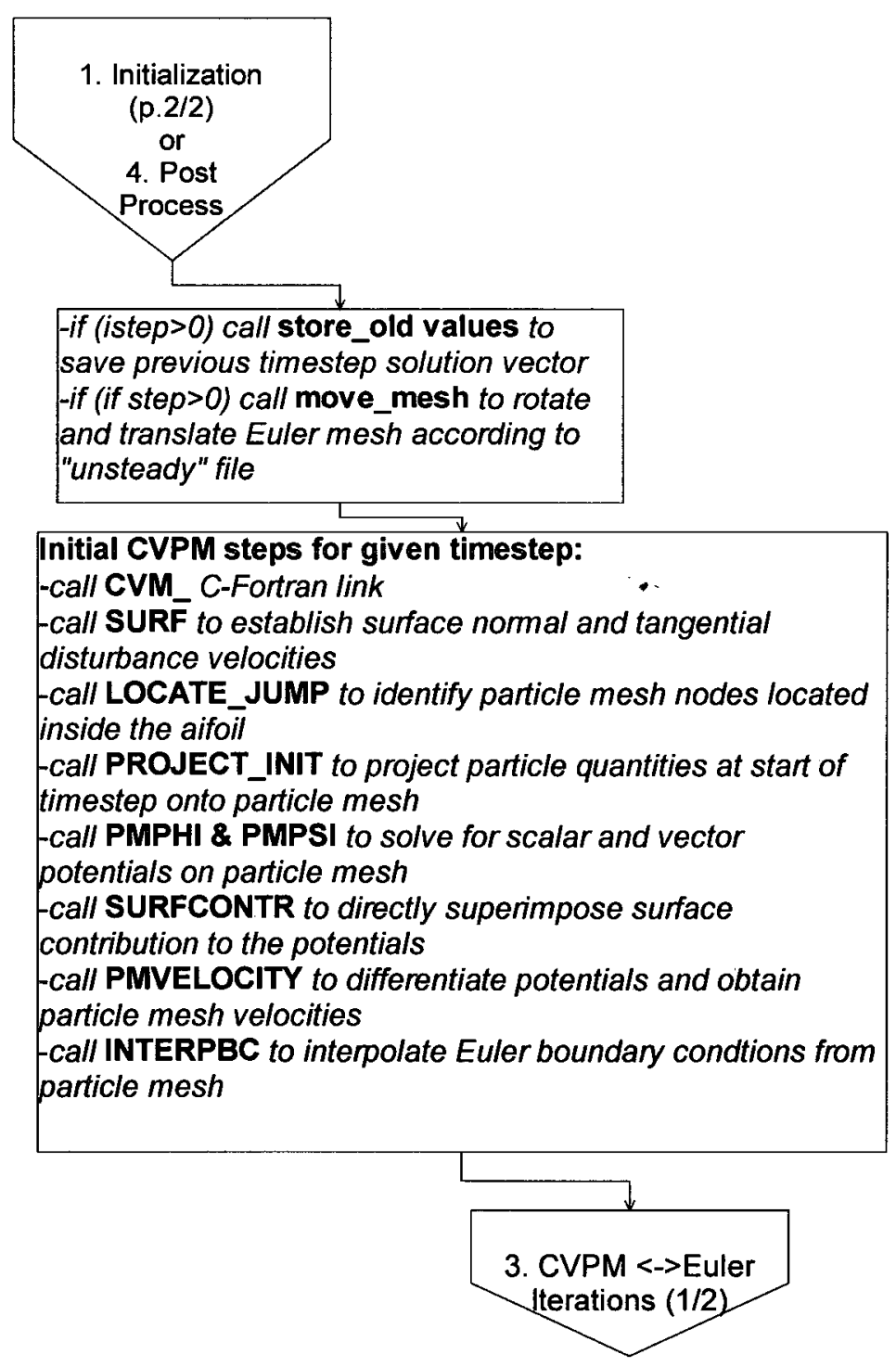


3a. CVPM <-> Euler Iterations (1/2)

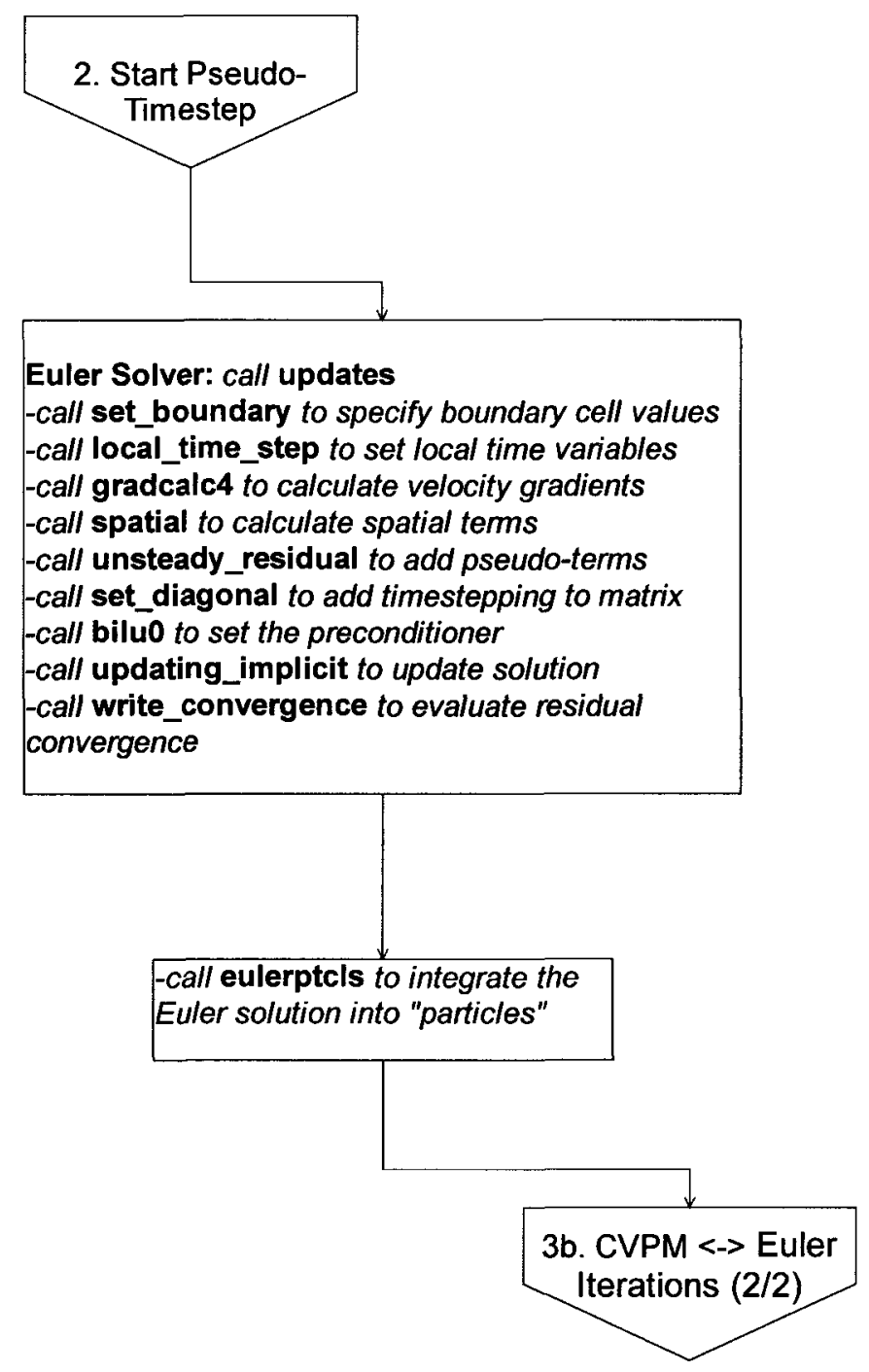


3b. CVPM <-> Euler Iterations (2/2)

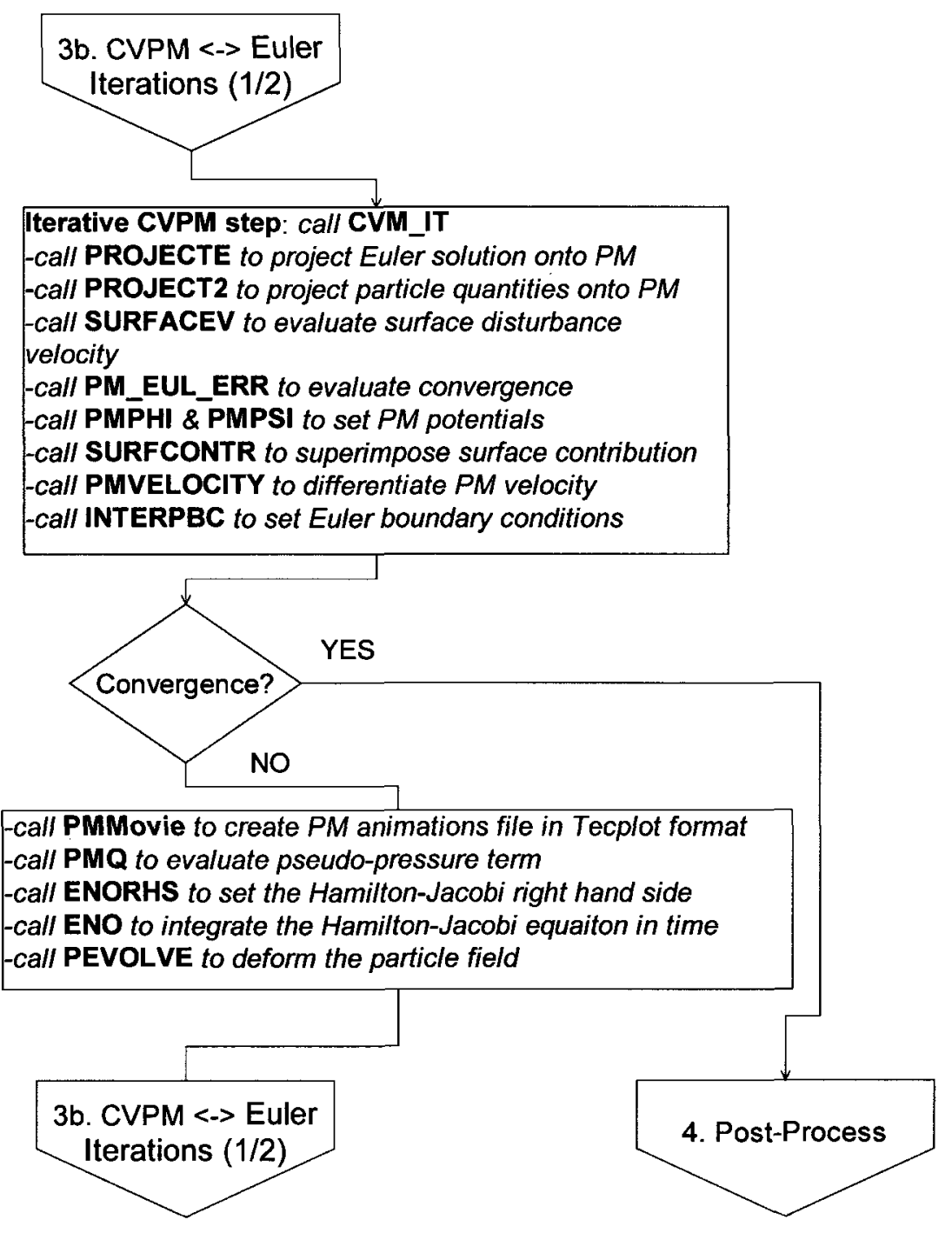




\section{POST-PROCESS}

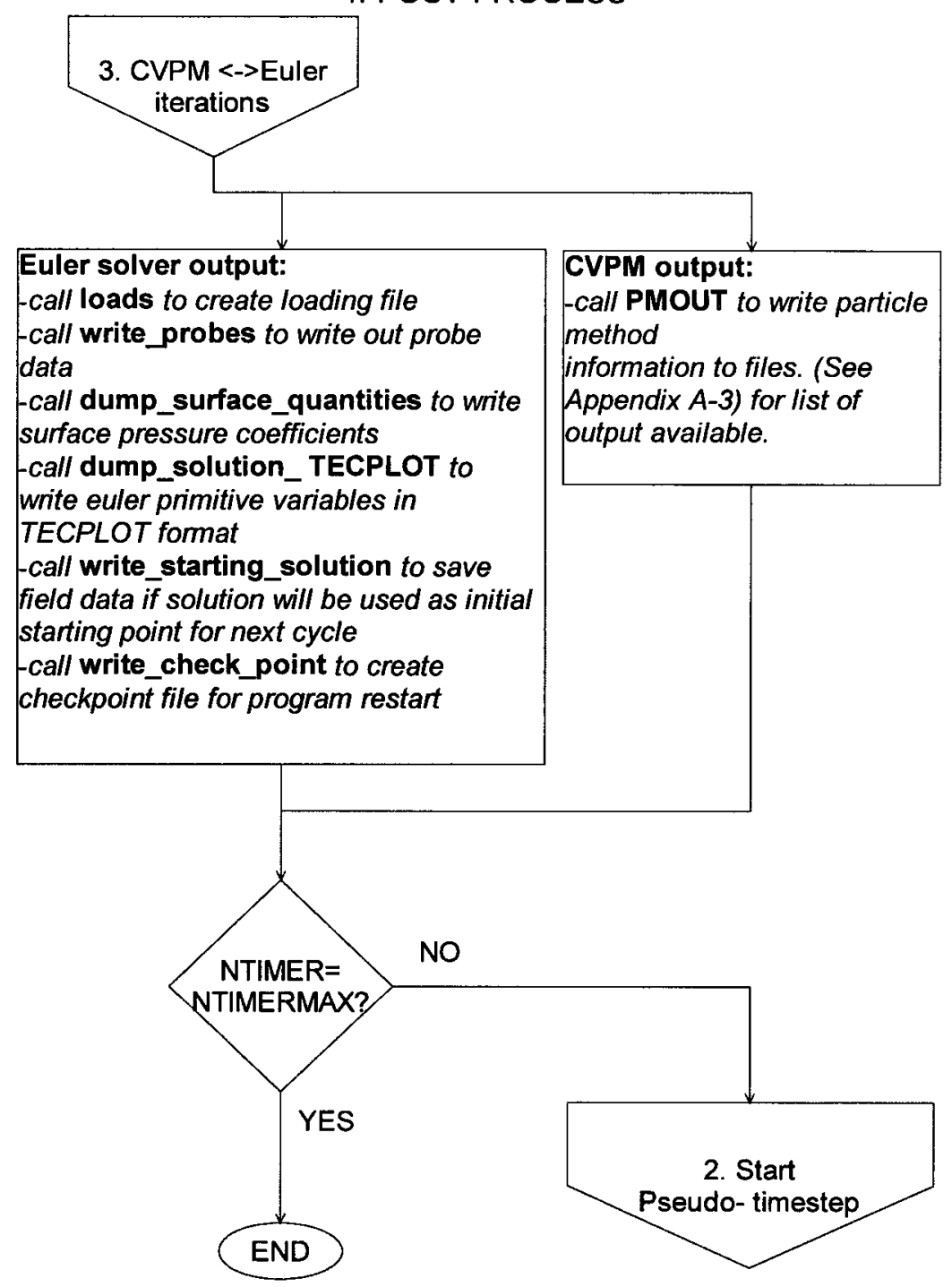

